\title{
A new hierarchically-structured $n$-dimensional covariant form of rotating equations of geophysical fluid dynamics
}

\author{
Werner Bauer \\ CliSAP/CEN (Center for Earth System Research and Sustainability) \\ University of Hamburg, Hamburg, Germany
}

6 May 2015

\begin{abstract}
We introduce $n$-dimensional equations of geophysical fluid dynamics (GFD) valid on rotating $n$-dimensional manifolds. Moreover, by using straight and twisted differential forms and an auxiliary velocity field, we introduce hierarchically-structured equations of (geophysical) fluid dynamics in which the equations are split into metric-free and metric-dependent parts. For these sets of equations we provide representations in local coordinate charts and we show that they conserve potential vorticity and that Kelvin's circulation theorem holds. As such general $n$-dimensional formulations do not exist in vector calculus, we provide for both covariant and vector-invariant equations a representation on a rotating coordinate frame in an Euclidean space and compare these representations. This study reveals, among others, that the prognostic variables, described by straight and twisted differential forms, are independent of both metric and orientation. This makes them perfect descriptors of the fluid's quantities of interest, as they assign, analogously to physical measurement devices, real valued numbers to finite distances, areas, or volumes. This is not the case for prognostic variables described by (vector) proxy fields, as they depend on metric and orientation.

The new structuring reveals also important geometrical features of the equations of GFD. For instance, the dimensioned differential forms display the geometric nature of the fluid's characteristics, while the equations' structuring illustrates how the metric-free momentum and continuity equations geometrically interact and how they are connected by the metricdependent Hodge star and Riemannian lift. Besides this geometric insight, this structuring has also practical benefits: since equations containing only topological structure are less complicated to implement and can be integrated exactly, our results may contribute to more efficient and accurate discretizations.
\end{abstract}

Keywords. Rotating Euler's equations, Covariant equations, Covariant split Euler's equations of GFD, Geophysical fluid dynamics, Differential geometry, and Exterior calculus

Mathematics Subject Classification (2010). Primary: 76U05, 76A02, 58J45; Secondary: 76M10, 53Z99.

*wbauer@physnet.uni-hamburg.de

This version has been accepted for publication in GEM - International Journal on Geomathematics. The final publication is available at Springer via http://dx.doi.org/10.1007/s13137-015-0074-8 


\section{Contents}

1 Introduction $\quad 2$

1.1 Content and structure of the manuscript . . . . . . . . . . . . . . . . . . . . 4

2 Mathematical spaces and descriptors for fluid flows $\quad \mathbf{5}$

2.1 Exterior Calculus and Differential Geometry in a nutshell . . . . . . . . . . . . . . . . . 5

2.2 Mathematical descriptors for displacement and force fields . . . . . . . . . . . . . . . . . . 12

3 The equations of GFD in vector-invariant form $\quad 15$

4 The equations of GFD in covariant form $r$

4.1 Derivation of covariant equations for ideal fluids using the master balance law . . . . . . . . . . 18

4.2 Derivation of covariant equations of GFD for the rotating earth . . . . . . . . . . . . . 20

5 Examples and Discussion of Part $1 \quad 22$

5.1 Rotating Cartesian coordinate frame on the sphere . . . . . . . . . . . . . . . . . 22

5.2 Representation of the covariant equations of GFD on a rotating Cartesian coordinate frame . . . . 23

5.3 Representation of the vector-invariant equations of GFD on a rotating Cartesian coordinate frame 25

5.4 Identities between representations of covariant and vector-invariant equations . . . . . . . . . . 26

5.5 Summary of Part $1 \ldots \ldots \ldots \ldots \ldots \ldots \ldots$

6 Covariant equations of GFD on rotating n-dimensional manifolds $\quad 28$

6.1 Representation of the covariant equations of GFD including the generalized apparent gravity . . . 32

7 Hierarchically-structured (split) covariant equations of GFD 33

7.1 Additionally required mathematical concepts and structures . . . . . . . . . . . . . . . . 33

7.2 Splitting the covariant equations into topological and metric parts . . . . . . . . . . . . . 35

7.3 Geometrical interpretation of the split Euler's equations of GFD . . . . . . . . . . . . 38

8 Conservation properties of the (split) covariant equations of GFD $\mathbf{4 4}$

8.1 The $n$-dimensional covariant vorticity equation . . . . . . . . . . . . . . . . . . . . . 44

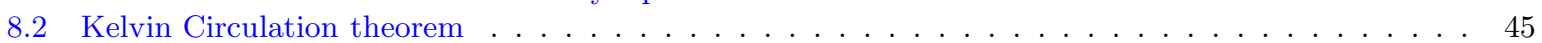

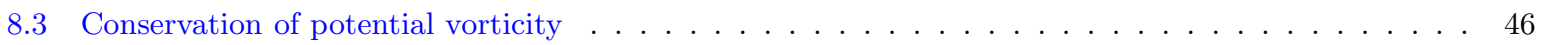

9 Examples and Discussion of Part $2 \quad 49$

9.1 Split shallow-water equations and their PV conservation . . . . . . . . . . . . . . . . . . . 49

9.2 Dependency of covariant and vector-invariant equations on metric and orientation . . . . . . . . 50

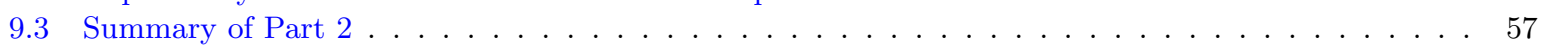

$\begin{array}{ll}10 \text { Conclusions and Outlook } & 57\end{array}$

\section{Introduction}

Most of the literature on geophysical fluid dynamics (GFD) proposes equations written in terms of classical vector calculus (see e.g. Durran (1999); Marshall and Plumb (2007); Pedlosky (1979)). In vector calculus one deals with vector fields defined in a 3-dimensional Euclidean space $E_{3}$. The use of vector identities enables abstract calculations, i.e. free of concrete coordinate representations, which makes calculations, proofs, and the resulting fluid equations valid for any vector representation in $E_{3}$ and invariant under changes in the coordinate system. Vector calculus enables hence more abstract and understandable formulations, whereas calculations, proofs, and the fluid equations in coordinate representations are often rather cumbersome and possibly only valid for a certain choice of coordinate system. However, as vector calculus applies the cross product, a generalization to higher dimensions or to general manifolds is not possible (Bossavit (1998b)). This restrictions usually transfer also to the discrete schemes such as in mimic discretization approaches (e.g. Perot et al. (2006)), in which the discrete operators mimic the analytical properties. 
The usage of exterior calculus and differential geometry (cf. Abraham et al. (1983); Jänich (2001)) allows us to overcome the drawbacks of vector calculus. In particular, these methods are not restricted to $E_{3}$, hence enable formulations on general $n$-dimensional manifolds (referred to as $n$-manifolds). This follows as dimensionless operators (such as the exterior derivative $\mathbf{d}$ which unifies grad, curl and div) act on differential forms (which carry information about their dimension and, possibly, orientation) that are used to describe the fluid's characteristics such as mass and velocity. Concepts of vector calculus are found in exterior calculus, in a general dimension-independent form, such as $\mathbf{d} \mathbf{d}=0$ in place of the vector calculus identities curl$\operatorname{grad}=0$ and div-curl $=0$. In addition, the large amount of vector calculus identities, often rather cumbersome to prove, condense in exterior calculus to same basic calculation rules.

Using differential geometry, the resulting so-called covariant equations are independent of dimension and their form is invariant under changes in coordinate system. In several publications, e.g. in Flanders (1963); Kambe (2002); Wilson (2011), such covariant equations for (non-rotating) fluids have been proposed. In particular, Abraham et al. (1983) introduced $n$ dimensional equations on (non-rotating) Riemannian $n$-manifolds for ideal incompressible and barotropic fluids. In Bauer (2013), we extended these equations to rotating covariant equations of GFD on rotating Riemannian $k$-manifolds $(k \leq 3)$ embedded in $\mathbb{R}^{3}$. In Holm et al. (2002), the authors derived a general covariant form of Euler-Poincaré equations and used it to derive 3-dimensional vector-invariant equations of GFD.

In literature, both fluid dynamics and geophysical fluid dynamics apply such covariant formulations to derive structure-preserving discretizations, where analytical conservation properties are automatically fulfilled in the discrete case. Such property would be, for instance, the Helmholtz decomposition where discrete vector fields consist, just as the analytical ones, in divergence-free and rotation-free parts. To discretize general partial differential equations (PDEs), the framework of "discrete exterior calculus (DEC)" has been developed using methods of algebraic topology (Hatcher (2002)). This framework is built on discrete versions of manifolds, differential forms and operators that mimic fundamental properties of their continuous counterparts (see e.g. Desbrun et al. (2005a,b); Hirani (2003); Mullen et al. (2011); Bochev and Hyman (2006)). Alternatively, by combining the theories of functional analysis, homology and exterior calculus, Arnold et al. $(2006,2010)$ have developed the framework of finite element exterior calculus (FEEC). FEEC structures various finite element spaces in a geometrical consistent way and provides a mathematical framework for derivations and proofs in order to derive consistent and stable discretizations. Using FEEC, Arnold and colleagues thus derived suitable mixed finite element pairs for various PDEs (e.g. Arnold (2013); Arnold et al. (2010)).

In computational fluid dynamics, only few studies (e.g. Desbrun et al. (2005b); Elcott et al. (2007); Pavlov et al. (2011); Wilson (2011)) apply DEC or FEEC to discretize covariant fluid equations. In geophysical fluid dynamics, where additional effects caused by Coriolis and centripetal forces have to be considered (Marshall and Plumb (2007)), we are aware of a few studies based on covariant shallow-water equations, but of none for the full 3-dimensional covariant equations. For instance, applying DEC, Simarro (2007) introduced a triangular and Bauer (2013) a hexagonal C-grid discretization of the shallow-water equations implemented in the nextgeneration numerical weather prediction and climate model ICON (Bonaventura and Ringler (2005); Rípodas et al. (2009)). More recently, Cotter and Thuburn (2014) derived for geophysical applications from similar covariant equations various mixed finite element pairs using FEEC.

Our long term goal is to derive from general covariant equations of GFD structure-preserving discretizations. However, there exist to the best of our knowledge neither $n$-dimensional covariant equations of GFD on rotating $n$-manifolds nor corresponding discretization approaches. Such $n$-dimensional equations and discretization methods would be, however, desirable as these formulations are independent of dimension. In case equations are not $n$-dimensional, they might 
change with dimension, a fact which might be true for any proof conducted, and/or for the equations' (conservation) properties. For instance, discretization schemes suitable for two dimensions are often not generalizable to the 3-dimensional case. Besides such dimension-independent formulation, by using (straight and twisted) differential forms as descriptors for the fluid's quantities of interest, one can find a form of the equations that is independent of the manifold's orientation and therefore avoids the confusion that usually comes along in vector calculus when using axial and polar vectors (cf. Bossavit (1998b)).

In this paper we therefore derive $n$-dimensional covariant equations of GFD on rotating $n$-manifolds using a covariant master balance law. In addition, by using straight and twisted differential forms and an auxiliary velocity field, we introduce to the best of our knowledge new hierarchically-structured equations of fluid dynamics and GFD, in which the equations are split into metric-free and metric-dependent parts. These sets of equations are extensions of ideas developed in Bauer (2013) for rotating $k$-manifolds $(k \leq 3)$ in $\mathbb{R}^{3}$ to the $n$-dimensional case. We focus here on the analytical derivations of these equations in order to provide a profound basis for their structure-preserving discretization using DEC and/or FEEC, which will be subject of upcoming work.

The splitting of the equations will allow us to interpret the geometrical properties of the single terms of the split equations and their mutual interaction. Similarly structured sets of equations exist in literature: for instance, the split covariant Maxwell's equations introduced by Bossavit (2005) or the discrete shallow-water equations introduced by Cotter and Thuburn (2014). Moreover, this form of the equations suggests their structure-preserving discretization using DEC or FEEC, as introduced in Bauer (2013) for split linear shallow-water equations.

Finally, we show that these sets of equations are well-defined, prove that they conserve potential vorticity and that Kelvin's circulation theorem holds, and provide concrete $n$-dimensional representations in local coordinate charts. We assess identities and differences between covariant and vector-invariant equations for a representation in an Euclidean space, as only in the latter space vector-invariant equations exist. As we will furthermore show, these formulations behave differently under changes in coordinates, orientation or metric. We will discuss how these different behaviors impact on how well these sets of equations model "reality".

\subsection{Content and structure of the manuscript}

The paper consists of two major parts. Part 1 consisting of Sections 2 to 5 deals with the formulation and comparison of the equations of GFD in either covariant or vector-invariant form. In Sect. 2, we discuss suitable mathematical descriptors for the movement of fluid particles and for the forces acting upon these particles. We introduce the required mathematical spaces and operators in a hierarchical order, i.e. with respect to the mathematical structure required. In Sect. 3, we introduce vector-invariant equations of GFD and how these are derived using vector fields as descriptors. In Sect. 4, we derive the covariant equations of GFD on rotating general manifolds embedded in $\mathbb{R}^{3}$. In Sect. 5 , we represent covariant and vector-invariant equations on a rotating coordinate frame, which allows us to compare both representations, and we discuss similarities and differences.

In Part 2, consisting of Sections 6 to 9, we introduce and discuss formulations of the equations of GFD that would not exist in vector calculus. In Sect. 6, we introduce with Theorem 2 the first major result of this paper, namely to the best of our knowledge new $n$-dimensional covariant equations of GFD on a rotating $n$-dimensional manifold. In Sect. 7 , we introduce with Theorem 3 the second major result, namely to the best of our knowledge new hierarchically-structured fluid equations that are split into metric-free and metric-dependent parts. This structure results by describing the quantities of interest by straight and twisted differential forms, which will be 
introduced, alongside with additional mathematical structure, in Sect. 7.1. In Sect. 8, Theorem 4 and Theorem 5 are further major results which show that the (split) covariant equations conserve potential vorticity and that Kelvin's circulation theorem holds. In Sect. 9, we show for the split covariant shallow-water equations the conservation of potential vorticity, and we show explicitly how differential forms or vector fields used to describe the prognostic variables and the corresponding covariant or vector-invariant equations transform under changes in metric and orientation. In Sect. 10, we draw conclusions and provide an overview as on how the results of this paper might impact on future research.

\section{Mathematical spaces and descriptors for fluid flows}

In Part 1 of the manuscript, we derive covariant equations of geophysical fluid dynamics for general manifolds embedded in $\mathbb{R}^{3}$. Then, we compare these equations with the vector-invariant ones, in a suitable coordinate representation. By the choice of adequate mathematical descriptors for the fluid motion and the forces involved, these equations follow from balance laws.

In this section, we first introduce the required spaces and operators of differential geometry and then discuss adequate mathematical descriptors for the fluid motion and for the forces acting upon the fluid particles.

\subsection{Exterior Calculus and Differential Geometry in a nutshell}

We introduce the spaces, manifolds, and corresponding operators in a hierarchical order, i.e. from general topological spaces, on which vector fields and differential forms are well-defined, to more structured oriented Riemannian manifolds that are equipped with metric and orientation. Moreover, we discuss operators that are defined on and that build connections between these spaces. Throughout Section 2, we present the mathematical spaces and operators as usually introduced in textbooks (e.g. Abraham et al. (1983); Bossavit (2005); Jänich (2001)). For more details on their definitions, properties and proofs we refer to these references.

\subsubsection{Topological manifolds}

A topological space is the most general mathematical space that enables to define the notions of connectedness, continuity, and convergence. It consists of a set of points and of a topology that is a set of neighborhoods (or open sets) to each point satisfying certain axioms (Abraham et al. (1983)). An $n$-dimensional topological manifold $\mathcal{M}$ (called topological $n$-manifold) is a topological space covered with open sets $U_{i} \subset \mathcal{M}, \mathcal{M}=\cup_{i \in I} U_{i}$, such that each $p \in \mathcal{M}$ lies in at least one $U_{i}$, and equipped with a set of homeomorphisms $\phi_{i}: U_{i} \rightarrow V \in \mathbb{R}^{n}$ (cf. Kambe (2002)). The pair $\left(U_{i}, \phi_{i}\right)$ defines coordinate patches on $\mathcal{M}$ and makes the manifold locally, in the neighborhood $U$ of each $p$, look like an $n$-dimensional affine space $A_{n}$. Differentiable manifolds are those for which the overlap function $\phi_{j} \circ \phi_{i}^{-1}, i, j \in I$, for any pair of coordinate charts $\left(U_{i}, \phi_{i}\right)$ and $\left(U_{j}, \phi_{j}\right)$ is a $C^{\infty}$-map from $\phi_{i}\left(U_{i} \cap U_{j}\right)$ to $\phi_{j}\left(U_{i} \cap U_{j}\right)$ in $\mathbb{R}^{n}$. We call the family of such coordinate charts $\left(U_{i}, \phi_{i}\right)_{i \in I}$ an atlas on $\mathcal{M}$; and we call it a complete (or maximal) atlas, if it is not already contained in any other atlas.

Given this atlas, functions $f: \mathcal{M} \rightarrow \mathcal{N}$ from the $n$-manifold $\mathcal{M}$ to the $l$-manifold $\mathcal{N}$ can be locally represented with respect to the coordinate charts $\left(U_{1} \subset \mathcal{M}, \phi_{1}\right)$ and $\left(U_{2} \subset \mathcal{N}, \phi_{2}\right)$ by $\phi_{2} \circ f \circ \phi_{1}^{-1}: \phi(U): \mathbb{R}^{n} \rightarrow \mathbb{R}^{l}$. Properties valid for functions of the form $\mathbb{R}^{n} \rightarrow \mathbb{R}^{l}$ can thus be transfered to differentiable manifolds. For instance, $f$ is a $C^{r}$ function if, for all coverings of $\mathcal{M}$ and $\mathcal{N}$, the local representatives are $C^{r}$ functions. Similarly, the projection functions $u^{k}: \mathbb{R}^{n} \rightarrow \mathbb{R}, u^{k}(x):=x^{k}, k=1, \ldots, n$, that assign to a point $x \in \mathbb{R}^{n}$ its coordinates $\left(x^{1}, \ldots, x^{n}\right)$ 
can be used to define the coordinate functions $\phi^{k}: U \rightarrow \mathbb{R}, k=1, \ldots, n$, for a given chart $\left(U, \phi_{U}\right)$ by $\phi^{k}(p):=u^{k}\left(\phi_{U}(p)\right)$. Then, the point $p \in U \subset \mathcal{M}$ has the coordinates $\left(\phi^{1}(p), \ldots, \phi^{n}(p)\right)$. As frequently done (e.g. in the Ricci calculus), we write simply $x^{k}$ for the coordinate functions, thus presenting the coordinates of $p$ by the $n$-tuple of real numbers $\left(x^{1}(p), \ldots, x^{n}(p)\right)$.

Moreover, we need the concept of tangent spaces, which are spanned by tangent vectors of curves on the manifold $\mathcal{M}$. A curve on $\mathcal{M}$ is a smooth map $\gamma: I \rightarrow \mathcal{M}$ from some interval $I \in \mathbb{R}$ into $\mathcal{M}$. Let the set of such curves that pass point $p \in \mathcal{M}$ at $t=0$ be denoted by $\mathcal{K}_{p}(\mathcal{M})=\{\gamma:(-\epsilon, \epsilon) \rightarrow \mathcal{M} \mid \epsilon>0$ and $\gamma(0)=p\}$. Two curves $\gamma_{1}, \gamma_{2} \in \mathcal{K}_{p}(\mathcal{M})$ are called tangential equivalent, $\gamma_{1} \sim \gamma_{2}$, if for one (thus any) chart $(U, \phi)$ around $p$ there is: $\frac{d}{d t}(\phi \circ$ $\left.\gamma_{1}(t)\right)\left.\right|_{t=0}=\left.\frac{d}{d t}\left(\phi \circ \gamma_{2}(t)\right)\right|_{t=0} \in \mathbb{R}^{n}$. The equivalence classes $[\gamma] \in \mathcal{K}_{p}(\mathcal{M}) / \sim$ are denoted as tangent vectors of $\mathcal{M}$ at $p$ and the vector space $\mathcal{T}_{p} \mathcal{M}:=\mathcal{K}_{p}(\mathcal{M}) / \sim$ is called the tangent space of $\mathcal{M}$ at $p$. The tangent spaces allow us to define the differential of a function $f: \mathcal{M} \rightarrow \mathcal{N}$ at point $p$ by the linear mapping of tangent vectors: $d f_{p}: \mathcal{T}_{p} \mathcal{M} \rightarrow \mathcal{T}_{f(p)} \mathcal{N},[\gamma] \mapsto[f \circ \gamma]$ (see e.g. Jänich (2001)).

The union of tangent spaces on $\mathcal{M}$ defined by $\mathcal{T} \mathcal{M}:=\cup_{p \in \mathcal{M}} \mathcal{T}_{p} \mathcal{M}$ is called the tangent bundle. $\mathcal{T} \mathcal{M}$ is a smooth $2 n$-dimensional manifold. Moreover, the projection $\pi: \mathcal{T} \mathcal{M} \rightarrow \mathcal{M}$, with $\pi(\zeta)=p$ in case $\zeta \in \mathcal{T}_{p} \mathcal{M}$, is a smooth mapping with $\pi^{-1}(p)=\mathcal{T}_{p} \mathcal{M}$. This enables the following definition.

Definition 1. A vector field $X \in \mathcal{X}(\mathcal{M})$ on an $n$-manifold $\mathcal{M}$ is a smooth cross-section in the tangent bundle $\mathcal{T} \mathcal{M}$ by

$$
X: \mathcal{M} \rightarrow \mathcal{T} \mathcal{M} \text { with } \pi(X(p))=p \text { for all } p \in \mathcal{M} .
$$

In other words, a vector field is a smooth assignment $\mathcal{M} \ni p \mapsto X_{p} \in \mathcal{T}_{p} \mathcal{M}$ for all $p \in \mathcal{M} . \mathcal{X}(\mathcal{M})$ denotes the space of vector fields on $\mathcal{M}$.

On a coordinate chart $(U, \phi)$, such vector field $X$ can be expanded on $U$ in the basis $\left.\frac{\partial}{\partial x^{k}}\right|_{p} \in$ $\mathcal{T}_{p} \mathcal{M}, k=1, \ldots, n$, for all $p \in U \subset \mathcal{M}$ by

$$
X=\sum_{k=1}^{n} X^{k} \frac{\partial}{\partial x^{k}},
$$

where $\frac{\partial}{\partial x^{k}}:\left.p \mapsto \frac{\partial}{\partial x^{k}}\right|_{p}$ for all $p \in U$ are vector fields on $U$ and $X^{k}$ are real functions on $U$.

Remark 1. Besides introducing coordinates on $U$ by $\phi^{k}: U \rightarrow \mathbb{R}$, a coordinate chart $(U, \phi)$ assigns also to every point $p \in U$ a basis for the tangent space $\mathcal{T}_{p} \mathcal{M}$ that corresponds under the mapping $\phi: \mathcal{T}_{p} \mathcal{M} \rightarrow \mathbb{R}^{n}$ to the canonical basis $\left(e_{1}, \ldots, e_{n}\right)$ of $\mathbb{R}^{n}$. The corresponding tangent vectors $\frac{\partial}{\partial x^{k}}$ of $\mathcal{T}_{p} \mathcal{M}$ are represented by the curves $t \mapsto \phi^{-1}\left(\phi(p)+t e_{k}\right)$, i.e. $\frac{\partial}{\partial x^{k}}:=$ $\left(d \phi^{-1}\right)\left[\phi(p)+t e_{k}\right]$. Alternatively, as derivatives acting on functions $f$, they are defined by $\left(\frac{\partial}{\partial x^{k}}\right)_{p} f:=\left.\frac{\partial}{\partial \phi^{k}(p)} f \circ \phi^{-1}\right|_{\phi(p)}, k=1, \ldots, n$.

On the tangent spaces, or vector spaces in general, we can define multilinear maps as follows.

Definition 2. Given a real vector space $V$, a $k$-form $\omega$ on $V$ is a skew-symmetric multilinear map

$$
\omega: \underbrace{V \times \cdots \times V}_{k-\text { times }} \longrightarrow \mathbb{R}
$$

with $\omega\left(v_{1}, \ldots, v_{k}\right)=0$ in case $v_{1}, \ldots, v_{k} \in V$ are linearly dependent. We denote the vector space of $k$-forms on $V$ by $\Lambda^{k}(V)$. For $k=0$, we set $\Lambda^{0}(V):=\mathbb{R}$. 
If $\alpha \in \Lambda^{k}(V)$ and $\beta \in \Lambda^{l}(V)$, we define the exterior (or wedge) product $\alpha \wedge \beta \in \Lambda^{k+l}$ by

$$
\alpha \wedge \beta=\frac{(k+l) !}{k ! l !} \mathbf{A}(\alpha \otimes \beta),
$$

in which $\otimes$ is the tensor product and $\mathbf{A}$ an alternating map, i.e. if two entries of $\mathbf{A}$ are exchanged, the sign changes. This product has the following properties (Abraham et al. (1983)):

(i) $\alpha \wedge \beta=\mathbf{A} \alpha \wedge \beta=\alpha \wedge \mathbf{A} \beta$,

(ii) $\wedge$ is bilinear,

(iii) $\alpha \wedge \beta=(-1)^{k+l} \beta \wedge \alpha$,

(iv) $\alpha \wedge(\beta \wedge \gamma)=(\alpha \wedge \beta) \wedge \gamma$.

The direct sum of the spaces $\Lambda^{k}(V), k=1, \ldots, n$, i.e. $\Lambda(V):=\oplus_{k=0}^{n} \Lambda^{k}(V)$, together with its structure as a real vector space and multiplication induced by the wedge product $\wedge$, is called the exterior algebra (or Grassmann algebra) of $V$.

Using this multiplication, the general $k$-forms of (3) can be represented with a basis in $\Lambda(V)$. To this end, we consider for a basis $\left(e_{1}, \ldots, e_{n}\right)$ of $V$, the dual basis $\left(e^{1}, \ldots, e^{n}\right)$ of the dual space $V^{*}$ which is defined by $e^{i}\left(e_{j}\right)=\delta_{i j}$. Then, the basis of $\Lambda^{k}(V)$ is given by $\left(\begin{array}{l}n \\ k\end{array}\right)$ basis vectors $\left\{e^{\mu_{1}} \wedge \cdots \wedge e^{\mu_{k}} \mid 1 \leq \mu_{1}<\cdots<\mu_{k} \leq n\right\}$ with $e^{\mu_{1}} \wedge \cdots \wedge e^{\mu_{k}}\left(e_{\mu_{1}}, \ldots, e_{\mu_{k}}\right)=1$ $\forall 1 \leq \mu_{1}<\cdots<\mu_{k} \leq n$ and 0 otherwise. In this basis, every $k$-form $\omega \in \Lambda^{k}(V)$ can be written as

$$
\omega=\sum_{1 \leq \mu_{1}<\cdots<\mu_{k} \leq n} \omega_{\mu_{1} \ldots \mu_{k}} e^{\mu_{1}} \wedge \cdots \wedge e^{\mu_{k}}
$$

with components $\omega_{\mu_{1} \ldots \mu_{k}}:=\omega\left(e_{\mu_{1}}, \ldots, e_{\mu_{k}}\right)$.

Such multilinear maps exist also in affine spaces, because a pointwise definition of $k$-forms $\left.\omega\right|_{x}$ on vector spaces $V_{x}$ at every point $x$ can be introduced. For instance, for $k=1$ and a given $x$, Definition 2 includes the description of forces by covectors (linear maps) $\left.\omega\right|_{x}=\mathcal{F}_{x}$ as elements of the dual space $\Lambda^{1} V_{x}=V_{x}^{*}$ (cf. discussion in Sect. 2.2.2). Analogously to affine spaces, for every point $p \in \mathcal{M}$ such multilinear maps $\omega_{p}$ exist on the tangent spaces $\mathcal{T}_{p} \mathcal{M}=V_{p}$, as the latter are vector spaces. The corresponding dual spaces, called cotangent spaces to the tangent spaces $\mathcal{T}_{p} \mathcal{M}$, will be denoted with $\mathcal{T}_{p}^{*} \mathcal{M}=V_{p}^{*}$.

Similarly to tangent bundles, there exist cotangent bundles defined by $\mathcal{T}^{*} \mathcal{M}:=\cup_{p \in \mathcal{M}} \mathcal{T}_{p}^{*} \mathcal{M}$. This allows us to define the bundle of $k$-forms by $\Lambda^{k}(\mathcal{T} \mathcal{M}):=\cup_{p \in \mathcal{M}} \Lambda^{k}\left(\mathcal{T}_{p} \mathcal{M}\right)$ with projection $\pi: \Lambda^{k}(\mathcal{T} \mathcal{M}) \rightarrow \mathcal{M}$ and with $\pi\left(\omega_{p}\right)=p$ in case $\omega_{p} \in \Lambda^{k}\left(\mathcal{T}_{p} \mathcal{M}\right)$. In turn, the dual spaces of the tangent spaces are spanned by $\pi^{-1}(p)=\mathcal{T}_{p}^{*} \mathcal{M}$. This enables the following general definition.

Definition 3. A differential form of grade $k$, or short (smooth) $k$-form, $\omega \in \Omega^{k}(\mathcal{M})$, is a differentiable map

$$
\omega: \mathcal{M} \rightarrow \Lambda^{k}(\mathcal{T} \mathcal{M}) \text { with } \pi(\omega(p))=p \text { for all } p \in \mathcal{M} .
$$

In other words, a differential form is a smooth mapping $\omega: \mathcal{M} \ni p \mapsto \omega_{p} \in \Lambda^{k}\left(\mathcal{T}_{p} \mathcal{M}\right)$. We denote the vector space of differentiable $k$-forms on $\mathcal{M}$ with $\Omega^{k}(\mathcal{M})$. For $k=0$, there is $\Omega^{0}(\mathcal{M})=C^{\infty}(\mathcal{M})$.

Similarly to the wedge product (4) on $V$, there exists a wedge product of differential forms on $\mathcal{M}$ by $\wedge: \Omega^{k}(\mathcal{M}) \times \Omega^{l}(\mathcal{M}) \rightarrow \Omega^{k+l}(\mathcal{M}),(\omega, \eta) \mapsto \omega \wedge \eta$, defined pointwise by $(\omega \wedge \eta)_{p}:=\omega_{p} \wedge \eta_{p}$ for all $p \in \mathcal{M}$. This wedge product on $\mathcal{M}$ shares the properties of (4), and gives also rise to the algebra $\Omega:=\oplus_{k=0}^{n} \Omega^{k}(\mathcal{M})$.

Analogously to $k$-forms on $V$, smooth $k$-forms on $\mathcal{M}$ can be locally represented in the form (5) using coordinate charts. We first consider smooth 1 -forms. On the coordinate chart $(U, \phi)$ with 
$\phi=\left(x^{1}, \ldots, x^{n}\right)$, the differentials $d x^{\mu}: \mathcal{T}_{p} \mathcal{M} \rightarrow \mathbb{R}$ of the coordinate functions $x^{\mu}: U \rightarrow \mathbb{R}$ map the basis vectors $\left(\partial_{1}, \ldots, \partial_{n}\right)$ of $\mathcal{T}_{p} \mathcal{M}$ like

$$
d x^{\mu}\left(\partial_{\nu}\right)=\frac{\partial x^{\mu}}{\partial x^{\nu}}=\delta_{\mu \nu}:=\left\{\begin{array}{l}
1 \text { if } \mu=\nu \\
0 \text { if } \mu \neq \nu .
\end{array}\right.
$$

These differentials build at every point $p \in U$ for the basis $\left(\partial_{1}, \ldots, \partial_{n}\right)_{p}$ the dual basis $\left(d x^{1}, \ldots, d x^{n}\right)_{p}$ of $\mathcal{T}_{p}^{*} \mathcal{M}$. Moreover, the differentials $d x^{\mu}$ return the $\mu$-component of a vector $X \in \mathcal{T}_{p} \mathcal{M}$ by $d x^{\mu}(X)=\sum_{\nu} X^{\nu} d x^{\mu}\left(\partial_{\nu}\right)=X^{\mu}$. Using this dual basis, a smooth 1-form $\omega$ can be represented in local coordinates $(U, \phi)$ by $\omega \mid U=\sum_{\mu} \omega\left(\partial_{\mu}\right) d x^{\mu}$.

In order to represent smooth $k$-forms, one applies the wedge product and the fact that differential forms induce a smooth mapping: $\omega: \mathcal{X}(\mathcal{M}) \times \ldots \times \mathcal{X}(\mathcal{M}) \longrightarrow C^{\infty}(\mathcal{M})$. Then, in a coordinate chart $(U, \phi)$, smooth $k$-forms can be represented by

$$
\omega=\sum_{1 \leq \mu_{1}<\cdots<\mu_{k} \leq n} \omega_{\mu_{1} \ldots \mu_{k}} d x^{\mu_{1}} \wedge \cdots \wedge d x^{\mu_{k}}
$$

using the component functions: $\omega_{\mu_{1} \ldots \mu_{k}}=\omega\left(\partial_{\mu_{1}}, \ldots, \partial_{\mu_{k}}\right): U \rightarrow C^{\infty}(U)$. In the following when there is no danger of confusion, smooth $k$-forms are frequently denoted simply as $k$-forms.

\subsubsection{Operators on topological manifolds acting on vector fields and differential forms}

The following operators acting on vector fields $\mathcal{X}(\mathcal{M})$ and on differential forms $\Omega(\mathcal{M})$ are defined on topological manifolds $\mathcal{M}$ and require a differentiable structure on $\mathcal{M}$.

Volume form We denote $\Omega=d x^{1} \wedge \cdots \wedge d x^{n} \in \Omega^{n}\left(\mathbb{R}^{n}\right)$ as the standard volume form on $\mathbb{R}^{n}$ with $\Omega(x) \neq 0 \forall x \in \mathbb{R}^{n}$. Analogously, a volume form on an $n$-manifold is a non-vanishing $n$-form $\mu \in \Omega^{n}(\mathcal{M})$ with $\mu(p) \neq 0$ for all $p \in \mathcal{M}$. Such volume form exists if and only if $\mathcal{M}$ is orientable (Abraham et al. (1983)).

There exist an infinite number of different volume forms, obtained by multiplying any volume form by a non-vanishing function. In case of an oriented Riemannian manifold, i.e. if a metric g exists (cf. Sect. 2.1.4), there is a natural volume form given in local coordinates by $\mu_{g}=$ $\sqrt{|\mathbf{g}|} d x^{1} \wedge \cdots \wedge d x^{n}$ with $\mu_{g}\left(\partial_{1}, \ldots, \partial_{n}\right)=1$.

Exterior derivative For a differentiable topological $n$-manifold $\mathcal{M}$, there is a unique family of mappings $\mathbf{d}^{k}: \Omega^{k}(\mathcal{M}) \rightarrow \Omega^{k+1}(\mathcal{M})(k=0, \ldots, n$, and $U$ is open in $\mathcal{M})$, denoted with exterior derivative $\mathbf{d}$, with the following properties (Abraham et al. (1983)):

(i) $\mathbf{d}$ is a $\wedge$-antiderivation, i.e. $\mathbf{d}$ is $\mathbb{R}$-linear and for $\alpha \in \Omega^{k}(\mathcal{M})$ and $\beta \in \Omega^{l}(\mathcal{M})$,

$$
\mathbf{d}(\alpha \wedge \beta)=\mathbf{d} \alpha \wedge \beta+(-1)^{k} \alpha \wedge \mathbf{d} \beta \quad \text { (product rule); }
$$

(ii) if $f \in \mathcal{C}^{\infty}(\mathcal{M}), \mathbf{d} f$ is the total differential of $f$, i.e. $(\mathbf{d} f)_{\mu}=\frac{\partial f}{\partial x^{\mu}}$ and $x^{\mu}, \mu=1, \ldots, n$, are local coordinates;

(iii) $\mathbf{d}^{2}=\mathbf{d} \circ \mathbf{d}=0$ (that is, $\left.\mathbf{d}^{k+1}(U) \mathbf{d}^{k}(U)=0\right)$;

(iv) $\mathbf{d}$ is a local operator, i.e. $\mathbf{d}$ is natural with respect to restrictions; that is, if $U \subset V \subset \mathcal{M}$ are open and $\alpha \in \Omega^{k}(V)$, then $\mathbf{d}(\alpha \mid U)=\mathbf{d} \alpha \mid U$. 
In a coordinate chart $(U, \phi), \phi=\left(x^{1}, \ldots, x^{n}\right)$, the exterior derivative of a smooth $k$-form $\omega \in \Omega^{k}(\mathcal{M})$ with representation (8) takes the form

$$
\mathbf{d} \omega=\sum_{1 \leq \mu_{1}<\cdots<\mu_{k} \leq n} \frac{\partial \omega_{\mu_{1} \ldots \mu_{k}}}{\partial x^{\mu}} d x^{\mu} \wedge d x^{\mu_{1}} \wedge \cdots \wedge d x^{\mu_{k}},
$$

with components $\omega_{\mu_{1} \ldots \mu_{k}}=\omega\left(\partial_{\mu_{1}}, \ldots, \partial_{\mu_{k}}\right): U \rightarrow C^{\infty}(U)$. For instance, for the 1-form $\omega^{1}=$ $\omega_{1} d x^{1}+\omega_{2} d x^{2} \in \Omega^{1}(\mathcal{M})$ in a 2 -manifold $\mathcal{M}$, there follows

$$
\mathbf{d} \omega=\partial_{2} \omega_{1} d x^{2} \wedge d x^{1}+\partial_{1} \omega_{2} d x^{1} \wedge d x^{2}=\left(\partial_{1} \omega_{2}-\partial_{2} \omega_{1}\right) d x^{1} \wedge d x^{2} .
$$

Remark 2. Be aware of the different notation between $\mathbf{d}$ and $d$, where $\mathbf{d}$ denotes the exterior derivative and $d$ the differential of the coordinate functions $x^{\mu}$.

Interior product (contraction) The inverse operation of the exterior derivative is the interior product (or contraction) $\mathbf{i}_{X}: \Omega^{k}(\mathcal{M}) \rightarrow \Omega^{k-1}(\mathcal{M})$ for all $k=1, \ldots, n$. The contraction of $\omega \in \Omega^{k+1}(\mathcal{M})$ by $X \in \mathcal{X}(\mathcal{M}), \mathbf{i}_{X} \omega \in \Omega^{k}(\mathcal{M})$, can be defined by

$$
\mathbf{i}_{X} \omega\left(X_{2}, \ldots, X_{k}\right)=\omega\left(X, X_{2}, \ldots, X_{k}\right),
$$

for all vectors $X_{i} \in \mathcal{X}(\mathcal{M}), i=2, \ldots, k$. If $\omega \in \Omega^{0}(\mathcal{M})$, we put $\mathbf{i}_{X} \omega=0$. The interior product has the following properties (Abraham et al. (1983)): if $\alpha \in \Omega^{k}(\mathcal{M}), \beta \in \Omega^{l}(\mathcal{M})$, and $f \in \Omega^{0}(\mathcal{M})$, then

(i) $\mathbf{i}_{X}$ is a $\wedge$-antiderivation, i.e. $\mathbf{i}_{X}$ is $\mathbb{R}$-linear and $\mathbf{i}_{X}(\alpha \wedge \beta)=\left(\mathbf{i}_{X} \alpha\right) \wedge \beta+(-1)^{k} \alpha \wedge\left(\mathbf{i}_{X} \beta\right)$,

(ii) $\mathbf{i}_{f X} \alpha=f \mathbf{i}_{X} \alpha$,

(iii) $\mathbf{i}_{X} \mathbf{d} f=\mathcal{L}_{X} f$

(iv) $\mathcal{L}_{X} \alpha=\mathbf{i}_{X} \mathbf{d} \alpha+\mathbf{d i}_{X} \alpha$

(v) $\mathcal{L}_{f X} \alpha=f \mathcal{L}_{X} \alpha+\mathbf{d} f \wedge \mathbf{i}_{X} \alpha$, where $\mathcal{L}$ denotes the Lie derivative, defined as follows.

Flows and Lie derivative We first introduce the notion of the flow $\varphi_{t}$ of a vector field $X$. This flow will be described by a one-parameter group of diffeomorphisms, i.e. the family $\left\{\varphi_{t}|t<| \epsilon \mid\right\}$ of local diffeomorphisms at $p \in U$ of the open set $U \subset \mathcal{M}$ onto the open set $\varphi_{t}(U)$, given by $\varphi:(-\epsilon, \epsilon) \times U \rightarrow \mathcal{M} ;(t, q) \mapsto \varphi_{t}(q), \forall q \in U$, fulfills the properties $\varphi_{s}\left(\varphi_{t}(q)\right)=\varphi_{s+t}(q)$ and $\varphi_{0}(q)=q$. At each $q \in U$ there is an integral curve $t \mapsto \varphi_{t}(q)$ inducing a tangent vector field $X$ by $q \mapsto X_{q}(f)=\left.\frac{d}{d t} f\left(\varphi_{t}(q)\right)\right|_{t=0}$ for all $q \in U$. The function $f$ could be any of the coordinate functions $x^{\mu}$, for instance. If $X$ is a complete vector field, the interval $(-\epsilon, \epsilon)$ can be extended to the entire real line (cf. Isham (1999)). We call $\varphi_{t}$ also the evolution operator.

On the basis of this flow description, we define the Lie derivative of a function (or tensor in general) as the rate of change of this function (or tensor) along the flow. Therefore, let $\varphi_{t}$ be the flow of a vector field $X \in \mathcal{X}(\mathcal{M})$ and $\tau \in T_{s}^{r}(\mathcal{M})$ be a tensor, both of class $C^{k}$. Then $\mathcal{L}_{X}$, called the Lie derivative with respect to $X$, is defined by

$$
\frac{d}{d t} \varphi_{t}^{*} \boldsymbol{\tau}=\varphi_{t}^{*} \mathcal{L}_{X} \boldsymbol{\tau}
$$

in which $t$ denotes the time variable and * the pull back (Abraham et al. (1983)). Using the Lie-bracket of vector fields [, ] $: \mathcal{X}(\mathcal{M}) \times \mathcal{X}(\mathcal{M}) \rightarrow \mathcal{X}(\mathcal{M})$, the Lie derivative $\mathcal{L}$ has the following properties (Abraham et al. (1983)): let $X, Y \in \mathcal{X}(\mathcal{M})$, then

(i) $\left[\mathcal{L}_{X}, \mathbf{i}_{Y}\right]=\mathbf{i}_{[X, Y]}$, 
(ii) $\left[\mathcal{L}_{X}, \mathcal{L}_{Y}\right]=\mathcal{L}_{[X, Y]}$,

(iii) $\mathbf{i}_{X} \circ \mathcal{L}_{X}=\mathcal{L}_{X} \circ \mathbf{i}_{X}$,

(iv) $\mathbf{d}$ is natural with respect to $\mathcal{L}_{X}$, i.e. for $\omega \in \Omega^{k}(\mathcal{M})$, there follow $\mathcal{L}_{X} \omega \in \Omega(\mathcal{M})^{k}$ and $\mathbf{d} \mathcal{L}_{X} \omega=\mathcal{L}_{X} \mathbf{d} \omega$.

Because of Cartan's formula (Eqn. (12) (iv)), there exists a metric-free form of the divergence of a vector field $X$ by

$$
\mathcal{L}_{X} \mu=\mathbf{i}_{X} \underbrace{\mathbf{d} \mu}_{=0}+\mathbf{d i}_{X} \mu=(\operatorname{div} X) \mu,
$$

using the volume form $\mu$ (Abraham et al. (1983)).

\subsubsection{Oriented topological manifolds and integration}

Equipping the topological manifold $\mathcal{M}$ with orientation $O r$ (cf. Sect. 7.1) enables to formulate integrals over forms and, in particular, to define a general version of Stokes' theorem. We denote oriented topological manifolds by $(\mathcal{M}, O r)$. On an oriented $n$-dimensional manifold, the integral over $n$-forms $\omega \in \Omega^{n}(\mathcal{M})$ is given in a coordinate chart $(U, h)$ by

$$
\int_{\mathcal{M}} \omega:=\int_{\mathbb{R}^{n}}\left(\omega_{1 \ldots n} \circ h^{-1}\right) d x^{1} \ldots d x^{n},
$$

with component function $\omega_{1 \ldots n}:=\omega\left(\partial_{1}, \ldots, \partial_{n}\right): U \rightarrow C^{\infty}(\mathcal{M})$ with compact support. Analogously, $k$-form can be integrated on $k$-dimensional submanifolds of $\mathcal{M}$. We will study the properties of this integral definition in more detail in Sect. 9.2.

The boundary of a topological $n$-manifold $\mathcal{M}$, denoted with $\partial \mathcal{M}$, is an $(n-1)$-dimensional topological manifold without boundary (Jänich (2001)). In general, a surface such as $\partial \mathcal{M}$ (possibly not connected) that encloses a manifold $\mathcal{M}$ can be consistently outer oriented by a consistent crossing direction from inside $\mathcal{M}$ to the outside (Bossavit (1998a)). In case of an oriented manifold $(\mathcal{M}, O r)$, this allows us to assign a consistent canonical inner orientation to the boundary $\partial \mathcal{M}$, namely by ordering first the outer normal on $\partial \mathcal{M}$ followed by the one inner orientation of $\partial \mathcal{M}$ that gives $\operatorname{Or}$ of $\mathcal{M}$ (cf. Bossavit (1998c)).

Let $\omega \in \Omega^{k-1}(\mathcal{M})$ be an $(n-1)$-form with compact support on an oriented $n$-dimensional manifold $\mathcal{M}$ with $(n-1)$-dimensional boundary $\partial \mathcal{M}$ that is canonically inner oriented. Then, Stokes' theorem is given by

$$
\int_{\mathcal{M}} \mathbf{d} \omega=\int_{\partial \mathcal{M}} \omega
$$

Stokes' theorem is also valid for twisted forms $\widetilde{\omega}$ of Definition 7 that act on outer oriented manifolds (cf. Sect. 7.3).

The exterior derivative, defined in Sect. 2.1.2, is a map of the form $\mathbf{d}: \Omega^{k}(\mathcal{M}) \rightarrow \Omega^{k+1}(\mathcal{M})$. As indicated by Stokes' theorem, the boundary operator acting on $k$-manifolds (or $k$-chains of Definition 6) is a continuous map $\partial_{k}: C_{k}(\mathcal{M}) \mapsto C_{k-1}(\mathcal{M})$ on the space of manifolds (or chains) (cf. Bossavit (2005)). As a consequence, the boundary of $\partial \mathcal{M}$ is null, or in general $\partial \partial=0$, which

follows directly by $\mathbf{d} \mathbf{d}=0$, because $\int_{\mathcal{M}} \mathbf{d} \mathbf{d} \omega=\int_{\partial \mathcal{M}} \mathbf{d} \omega=\int_{\partial \partial \mathcal{M}} \omega$. By linearity, the boundary operator acts on chains like $\partial\left(\sum_{i} \mu_{i} \mathcal{M}_{i}\right)=\sum_{i} \mu_{i} \partial \mathcal{M}_{i}$.

\subsubsection{Oriented topological manifolds with metric (Riemannian manifolds)}

Here, we equip (not necessarily oriented) topological manifolds (or affine spaces) with metric. Then, notions like length, area, volume, orthogonality (or angles in general) are well-defined. In 
addition, the metric structure allows us to define useful isomorphisms, such as an isomorphism between vector and covector fields.

To introduce a metric on topological $n$-manifolds, we first introduce it on vector spaces. To this end, let $V$ be an $n$-dimensional vector space and $\mathbf{g}=\langle\cdot, \cdot\rangle$ a nondegenerate symmetric bilinear form of rank $n$, i.e. the map $V \rightarrow V^{*}, \vec{v} \mapsto\langle\vec{v}, \cdot\rangle$, is an isomorphism. Then, there exists a g-orthonormal basis $\left(e_{1}, \ldots, e_{n}\right)$ of $V$ with dual basis $\left(e^{1}, \ldots, e^{n}\right)$ such that

$$
\mathbf{g}=\sum_{i=1}^{n} c_{i} e^{i} \otimes e^{i}, c_{i}= \pm 1 ; \quad \text { with matrix of } \mathbf{g}: \quad\left[\begin{array}{ccc}
c_{1} & & 0 \\
& \ddots & \\
0 & & c_{n}
\end{array}\right]
$$

The number of $c_{i}=-1$ in the diagonal of matrix $\mathbf{g}$ is called index $s:=\operatorname{Ind}(\mathbf{g})$. In case of $s=0$, the pair $(V,\langle\cdot, \cdot\rangle)$ is called Euclidean space $E_{n}$. More precisely, $E_{n}$ is an affine space $A_{n}$ where at each $x \in A_{n}$ the associated vector space $V_{x}$ is equipped with such a bilinear form $\langle\cdot, \cdot\rangle_{x}$.

The metric $\mathbf{g}$ induces a nondegenerate symmetric bilinear form, denoted with $\mathbf{g}^{(k)}=\langle\cdot, \cdot\rangle$, on the vector space of $k$-forms $\Lambda^{k}(V)$ : that is, if $\left(e_{1}, \ldots, e_{n}\right)$ is a $\mathbf{g}$-orthonormal basis of $V$, then $\left(e^{\mu_{1}} \wedge \ldots \wedge e^{\mu_{k}}\right)_{1 \leq \mu_{1}<\ldots<\mu_{k} \leq n}$ is a $\mathbf{g}$-orthonormal basis of $\Lambda^{k}(V)$ with respect to $\mathbf{g}^{(k)}$ and there is $\left\langle e^{\mu_{1}} \wedge \ldots \wedge e^{\mu_{k}}, e^{\mu_{1}} \wedge \ldots \wedge e^{\mu_{k}}\right\rangle=c_{\mu_{1}} \ldots c_{\mu_{k}}$.

With this we define: a semi-Riemannian manifold of index $s$ is a pair $(\mathcal{M},\langle\cdot, \cdot\rangle)$ consisting of a topological manifold $\mathcal{M}$ and a family

$$
\langle\cdot, \cdot\rangle=\left\{\langle\cdot, \cdot\rangle_{p}\right\}_{p \in \mathcal{M}}
$$

of symmetric nondegenerate bilinear forms $\langle\cdot, \cdot\rangle_{p}$ on $\mathcal{T}_{p} \mathcal{M}$ of index $s$, such that, for all charts $(U, h)$ of an atlas, the mapping $g_{\mu \nu}:=\left\langle\partial_{\mu}, \partial_{\nu}\right\rangle_{p}: U \rightarrow \mathbb{R}, p \mapsto\left\langle\partial_{\mu}, \partial_{\nu}\right\rangle_{p}$, for any vector field $\partial_{\mu}, \partial_{\nu} \in \mathcal{X}(\mathcal{M})$, is a $C^{\infty}(\mathcal{M})$ function. In case of $s=0$, the pair $(\mathcal{M},\langle\cdot, \cdot\rangle)$ is called Riemannian manifold. ${ }^{1}$

Also on semi-Riemannian manifolds a symmetric nondegenerate bilinear form $\mathbf{g}^{(k)}=\langle\cdot, \cdot\rangle$ exists, i.e. $\langle\cdot, \cdot\rangle: \Omega^{k}(\mathcal{M}) \times \Omega^{k}(\mathcal{M}) \rightarrow C^{\infty}(\mathcal{M})$ with $\langle\omega, \eta\rangle=\langle\eta, \omega\rangle$ for all $\omega, \eta \in \Omega^{k}(\mathcal{M})$.

As it becomes clear from the context, we use for all nondegenerate symmetric bilinear forms introduced so far the symbol $\langle\cdot, \cdot\rangle$; in particular we omit the $p$ in $\langle\cdot, \cdot\rangle_{p}$ if not necessary. Moreover, in case of $s=0$ we call $\langle\cdot, \cdot\rangle$ (or simply $\cdot$ ) an inner product. Only on spaces equipped with such inner product, the notion of length, angle, orthogonality, etc., makes sense.

Remark 3. There exist different realizations of such metric structures on Euclidean spaces and on Riemannian manifolds (see discussion in Sect. 9.2).

\subsubsection{Operators on (oriented) Riemannian manifolds}

Using a metric structure $\mathbf{g}$ on an $n$-manifold $\mathcal{M}$, we introduce in the following a mapping between 1 -forms and vector fields. Equipping this Riemannian $n$-manifold $(\mathcal{M}, \mathbf{g})$ with an orientation, also a mapping between $k$-forms and $(n-k)$-form can be established.

Flat and sharp operators A nondegenerate symmetric bilinear form $\langle\cdot, \cdot\rangle$ on a finite-dimensional vector space $V$ induces an isomorphism $V \cong V^{*}$ by the map $\vec{v} \mapsto\langle\vec{v}, \cdot\rangle$. On Riemannian manifolds, not necessarily oriented, such isomorphism also exists at every point $p \in \mathcal{M}$ between the tangent and cotangent spaces, $\mathcal{T}_{p} \mathcal{M} \cong \mathcal{T}_{p}^{*} \mathcal{M}$. We use the notation

$$
\mathcal{T}_{p} \mathcal{M} \underset{\sharp}{\stackrel{b}{\rightleftarrows}} \mathcal{T}_{p}^{*} \mathcal{M}
$$

\footnotetext{
${ }^{1}$ In case of index $\operatorname{Ind}(\mathbf{g})=1$, thus $c_{1}=-1, c_{2}=c_{3}=c_{4}=1$, one obtains a so-called Lorentz manifold.
} 
where $b: \vec{v} \mapsto\langle\vec{v}, \cdot\rangle$ is denoted as flat operator and we write $b(\vec{v})=\vec{v}^{b}$. The inverse operator $\sharp: \alpha:=\langle\vec{v}, \cdot\rangle \rightarrow \vec{v}$ is denoted as sharp operator and we write $\sharp(\alpha)=\alpha^{\sharp}$.

Because $\langle\cdot, \cdot\rangle$ is smooth on $\mathcal{M}$ according to Eqn. (18), $b$ and $\sharp$ act smoothly on smooth vector fields and $k$-forms on $\mathcal{M}$. That is, the isomorphism above exists also between 1 -form $\Omega^{1}(\mathcal{M})$ and vector fields $\mathcal{X}(\mathcal{M})$ with corresponding mappings

$$
b: \mathcal{X}(\mathcal{M}) \rightarrow \Omega^{1}(\mathcal{M}) \quad \text { and } \quad \sharp: \Omega^{1}(\mathcal{M}) \rightarrow \mathcal{X}(\mathcal{M}) .
$$

We denote in the following both mappings $b$ and $\sharp$ as Riemannian lift if there is no danger of confusion. Here it should be explicitly stressed that these operators do not depend on orientation as no such structure is involved in their definitions.

Hodge star operator Let $V$ be an oriented $n$-dimensional vector space and $\mathbf{g}=\langle\cdot, \cdot\rangle$ a nondegenerate symmetric bilinear form. Let $\mu$ be the volume form of $V$. Then, there exists a unique isomorphism, called Hodge star operator, $\star: \Lambda^{k}(V) \rightarrow \Lambda^{n-k}(V)$ satisfying

$$
\alpha \wedge \star \beta=\langle\alpha, \beta\rangle \mu \text { for } \alpha, \beta \in \Lambda^{k}(V) \text {. }
$$

The Hodge star operator has the following properties (Abraham et al. (1983)): let $\alpha, \beta \in \Lambda^{k}(V)$, then

(i) $\alpha \wedge \star \beta=\beta \wedge \star \alpha=\langle\alpha, \beta\rangle \mu$,

(ii) $\star 1=\mu, \star \mu=(-1)^{\operatorname{Ind}(\mathbf{g})}$,

(iii) $\star \star \alpha=(-1)^{\operatorname{Ind}(\mathbf{g})}(-1)^{k(n-k)} \alpha$,

(iv) $\langle\alpha, \beta\rangle=(-1)^{\operatorname{Ind}(\mathrm{g})}\langle\star \alpha, \star \beta\rangle$.

For a positive oriented $\mathbf{g}$-orthonormal basis $\left(e_{1}, \ldots, e_{n}\right)$ of $V$ and its dual basis $\left(e^{1}, \ldots, e^{n}\right)$, there is

$$
\star\left(e^{\sigma(1)} \wedge \ldots \wedge e^{\sigma(k)}\right)=c_{\sigma(1)} \ldots c_{\sigma(k)} \operatorname{sign}(\sigma)\left(e^{\sigma(k+1)} \wedge \ldots \wedge e^{\sigma(k+n)}\right),
$$

with $\sigma(1)<\ldots<\sigma(k)$ and $\sigma(k+1)<\ldots<\sigma(k+n)$, and permutation group $\sigma$ with $\operatorname{sign}(\sigma)=1$ for even and $\operatorname{sign}(\sigma)=-1$ for odd permutation. For instance, given in $\mathbb{R}^{3}$ a basis $\left(e_{1}, e_{2}, e_{3}\right)$, its dual $\left(e^{1}, e^{2}, e^{3}\right)$, and the coefficients $c_{1}=c_{2}=c_{3}=1$, the following relations hold: $\star e^{1}=e^{2} \wedge e^{3}$, $\star e^{2}=-e^{1} \wedge e^{3}$, and $\star e^{3}=e^{1} \wedge e^{2}$.

The Hodge star operator $\star$ generalizes to oriented Riemannian manifolds $(\mathcal{M}, \mathbf{g}, O r)$ via the definition over the tangent spaces, i.e. there follows the isomorphism $\star: \Omega^{k}(\mathcal{M}) \rightarrow \Omega^{n-k}(\mathcal{M})$ between smooth $k$ - and $(n-k)$-forms by $\alpha \wedge \star \beta=\langle\alpha, \beta\rangle \mu$ for $\alpha, \beta \in \Omega^{k}(\mathcal{M})$. This Hodge star operator on $\mathcal{M}$ shares Properties (i)-(iv) from Definition (21).

Remark 4. On an oriented Euclidean space $E_{3}$ consisting of a 3-dimensional oriented affine space with metric $\mathbf{g}$, a bilinear antisymmetric mapping, called the cross product, $\times: V \times V \rightarrow V$ can be defined. This operator will be required later on in order to represent vector-invariant equations such as in Eqn. (28). The cross product can also be represented by means of the operators $b$ and $\star$, i.e. $\vec{v} \times \vec{w}=\left[\star\left(\vec{v}^{b} \wedge \vec{w}^{b}\right)\right]^{\sharp}$ for $\vec{v}, \vec{w} \in V$ (see e.g. Abraham et al. (1983)).

The cross product allows us to assign to 2-forms $u \in \Omega^{2}(\mathcal{M})$ their corresponding vector proxies. That is, an alternating bilinear form, associated to a vector $\vec{u}:=u^{\sharp}$ can be found by $\{\vec{v}, \vec{w}\} \mapsto \vec{u} \cdot(\vec{v} \times \vec{w})$ for all $\vec{v}, \vec{w} \in V$ (cf. Bossavit (1998b)).

\subsection{Mathematical descriptors for displacement and force fields}

In this section, we introduce precise mathematical descriptors for particle displacements and fluid motion, and for forces and force fields that act upon the fluid particles. The descriptors should precisely describe the physical entities while employing only minimal mathematical structure. In this context, we are guided by the parsimony principle (Bossavit (2012)) suggesting that "a well structured theory will start with weak mathematical structures and will enrich them on demand". 

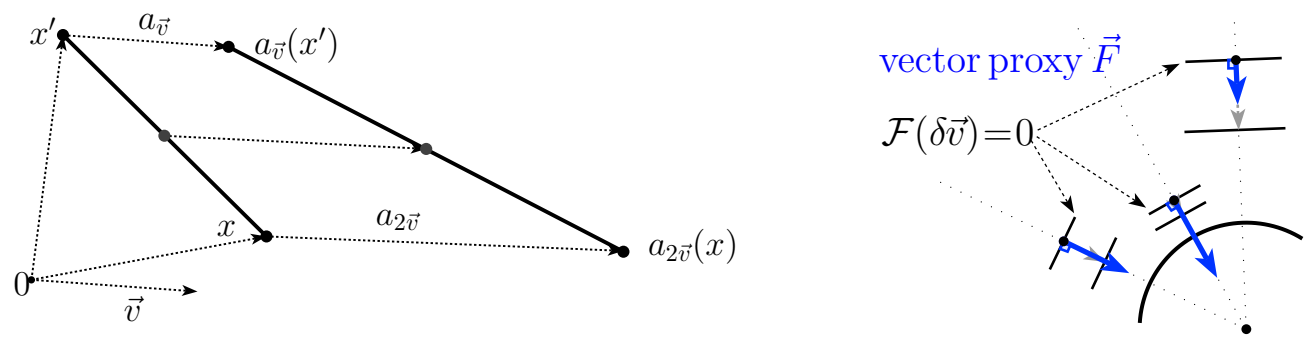

Figure 1: Left: affine translation $a_{\lambda \vec{v}}, \lambda \in[1,2]$, in $A_{n}$. Right: equipotential surfaces of covector field $\mathcal{F}$. The force field described by the vector proxy $\vec{F}$ is perpendicular to the equipotential surfaces of $\mathcal{F}$ (cf. Eqn. (23)).

\subsubsection{From displacement vectors to dynamic vector fields}

The physical displacement of a particle within a fluid from a given to a new position can adequately be characterized by two properties: (i) given an initial point, in principle, all new positions should be accessible, and (ii) the displacement has a direction and a magnitude.

An optimal mathematical descriptor for the particle displacement is provided by an affine translation $a_{\vec{v}}=x \rightarrow x+\vec{v}$ as element of an affine space $A_{n}$. This space consists of a set of points $x$ that admits a regular and transitive action of a vector space $V$ via $a_{\vec{v}}$. The affine translation is transitive, i.e. for any pair $\{x, y\}$ there is a vector $\vec{v}$ with $y=x+\vec{v}$, and is regular, i.e. for any $x$, there is $x+\vec{v} \neq x$ if $\vec{v} \neq 0$. As an affine map meets Conditions (i) and (ii), it is an optimal descriptor for particle displacement. Moreover, it conserves the alignment of points, leaves the barycenters and the ratio of distances between aligned points unchanged, and keeps parallel lines parallel; it does not however conserve quantities such as distances between points, orthogonality, or angles in general (see Fig. 1 (left)).

The displacements of all particles are described by the vector field $\vec{v}: A_{n} \rightarrow V_{x}, x \mapsto \vec{v}_{x} \forall x \in$ $A_{n}$, which assigns to every point $x \in A_{n}$ a tangent vector $\vec{v}_{x} \in V_{x}$. These displacement vectors are bound to the positions $x$ and, therefore, often denoted as bound vectors. ${ }^{2}$ In the dynamical case, the tangent vector field $x(t) \mapsto \vec{v}(t)$ describes the fluid particles' velocities at time $t$ and is a purely affine object. ${ }^{3}$

In affine spaces $A_{n}$, the particles' trajectories $x(t) \in A_{n}$ are lines, instead of general smooth curves, because of the linear character of affine spaces. The movement of fluid particles on general (possibly bent) surfaces can be optimally described by smooth (dynamic) vector fields $\vec{v}(t) \in \mathcal{X}(\mathcal{M})$ on topological manifolds $\mathcal{M}$. These vector fields assign smoothly to every particle at position $p \in \mathcal{M}$ a tangent vector $\vec{v}_{p} \in \mathcal{T}_{p} \mathcal{M}$ that describes the particle's infinitesimal displacement (or its velocity) caused by forces acting upon it. The affine transformations in $A_{n}$ can be regarded as local linearizations of such smooth vector fields on $\mathcal{M}$, because topological manifolds are locally isomorphic to affine spaces. At every point $A_{n} \ni x=p \in \mathcal{M}$, the affine transformations given by bound vectors $\vec{v}_{x} \in V_{x}$ correspond locally to the tangent vectors $\vec{v}_{p} \in \mathcal{T}_{p} \mathcal{M}$

\footnotetext{
${ }^{2}$ Bound vectors $\{x, \vec{v}(x)\}$ or $\{y, \vec{v}(y)\}$ are elements of the vector spaces $\left.V_{n}\right|_{x}$ at $x$ or $\left.V_{n}\right|_{y}$ at $y$, respectively. They form an affine space, because they can be added when regarded as pair of points, i.e. $\{x, x+\vec{v}(x)\}+\{y, y+$ $\vec{v}(y)\}=\{x+y, x+y+\vec{v}(x)+\vec{v}(y)\}=:\{z, \vec{v}(z)\}$, and multiplied by $\lambda \in \mathbb{R}$. However, bound vectors on different position (here in case of $x \neq y$ ) do not form a vector space (Bossavit (1998a)).

${ }^{3}$ Whereas vector fields are pure affine object without the concepts of metric and orientation, velocity fields, as presented, require the additional concept of a chronometer to measure time (Bossavit (1998b)).
} 


\subsubsection{From force covectors to differential forms}

A description of forces should cover the following properties: (i) forces are not directly visible: we experience them only by the effects they have on material, such as the displacements of particles; (ii) unlike displacements, forces do not have intrinsic pointing directions; (iii) through the displacements $\vec{v}$, force $F$ performs work $W=F \cdot|\vec{v}|$ on fluid particles (Bossavit (1998b)).

To describe forces by their effects (i.e. displacements) on particles, recall that we describe the particles' positions by $x$ as elements of the affine space $A_{n}$ and the particles' displacements by the bound vectors $\vec{v}_{x} \in V_{x}$, which span the vector space $V_{x}$. Then, an optimal descriptor for forces at point $x$ is given by linear maps (covectors)

$$
\mathcal{F}_{x}: V_{x} \rightarrow \mathbb{R}, \quad \vec{v}_{x} \mapsto \mathcal{F}_{x}\left(\vec{v}_{x}\right) \in \mathbb{R},
$$

as elements of the dual space $V_{x}^{*}$ of the vector space $V_{x}$. The idea is that forces assign to a virtual displacement an amount of virtual work. Such linear maps $\mathcal{F}_{x} \in V_{x}^{*}$ acting on displacements $\vec{v}_{x} \in V_{x}$ satisfy the Conditions (i)-(iii) imposed on forces and thus provide optimal descriptors for forces while only minimal mathematical structure is required.

Similarly to topological manifolds, the vector space $V_{x}$ corresponds to the tangent space of $A_{n}$ at point $x$, i.e. $V_{x}=T_{x} A_{n}$, hence $\mathcal{F}_{x}$ is an element of the cotangent space $V_{x}^{*}=T_{x}^{*} A_{n}$. An optimal descriptor for a (dynamical) force field is then given by the covector field $\mathcal{F}$, which is a linear map $\mathcal{F}: T A_{n} \rightarrow \mathbb{R}$ assigning a covector $\mathcal{F}_{x}$ to every point $x \in A_{n}$. This definition of forces as linear maps over affine spaces only requires an affine structure (i.e. no metric and orientation), analogously to the definition of affine translations.

Such piecewise linear covector fields $\mathcal{F}: \vec{v}_{x} \mapsto \mathcal{F}_{x}\left(\vec{v}_{x}\right), \forall x \in A_{n}$, act on piecewise linear vector fields $\vec{v}: x \mapsto \vec{v}_{x}, \forall x \in A_{n}$, and are therefore valid on piecewise linear lines, surfaces or volumes, as in case of simplicial complexes. Smooth force fields acting on smooth vector fields $\mathcal{X}(\mathcal{M})$ can be optimally described by smooth 1-forms $\omega \in \Omega^{1}(\mathcal{M})$ (i.e. smooth covector fields) on topological manifolds $\mathcal{M}$; at every point $p \in \mathcal{M}$, this force field smoothly assigns by the covector $\omega_{p} \in \mathcal{T}_{p}^{*} \mathcal{M}$ to a displacement $\vec{v}_{p} \in \mathcal{T}_{p} \mathcal{M}$ an amount of work $\omega_{p}\left(\vec{v}_{p}\right) \in \mathbb{R}$. Similarly, also other fluid's quantities of interest are optimally described by $k$-forms; for instance, a 2 -form assigns an amount of circulation to an area or a 3-form assigns an amount of mass to a volume. The latter fact will be subject of our discussion in Sect. 7.3. Similarly to the fact that affine spaces $A_{n}$ can be regarded as local linearizations of a manifold $\mathcal{M}$ around point $p \in \mathcal{M}$, the multilinear maps $\omega_{p} \in \Lambda(V)$ of Eqn. (3) can be regarded as local linearizations of the differential forms $\omega \in \Omega(\mathcal{M})$ of Definition 3 around point $p \in \mathcal{M}$.

Remark 5. On topological manifolds (or in affine spaces) force fields can be optimally described by smooth covector fields $\mathcal{F}$, which means that their definition requires minimal structure. The full information about the force field is encoded in the distribution of equipotential surfaces, as illustrated in Fig. 1 (right). These surfaces are approximated by parallel tangent planes, which are spanned by vectors $\delta \vec{v}$ that lie in the kernel of the linear maps $\mathcal{F}$, i.e. $\mathcal{F}(\delta \vec{v})=0$. The virtual work performed by virtual displacements $\delta \vec{v}$ that lie in these planes is zero. The distance between two parallel planes indicates the strength of the force field, where smaller distance means higher magnitude, or, in other words, the higher the density of the equipotential surfaces, the stronger is the force field. This description of force fields is valid on topological manifolds (or affine spaces) since it does not require length nor orthogonality, whereas the parallel planes remain parallel under affine transformations.

Remark 6. In case of Riemannian manifolds, the covector field (force field) $\mathcal{F}$ of Eqn. (23) can also be represented by its vector proxy field $\vec{F}$ defined by $\mathcal{F}=\vec{F}^{b}=\langle\vec{F}, \cdot\rangle$ using the Riemannian lift b. As illustrated in Fig. 1 (right), this force field $\vec{F}$ is at every point of the domain perpendicular to the equipotential surfaces of $\mathcal{F}$. As the notion of perpendicularity only exists in spaces with 
metric, vector proxy fields require such structure to describe forces. As there exists no unique metric structure on topological manifolds (or $A_{n}$ ), there exists also no unique vector proxy field to $\mathcal{F}$. In addition, the vector proxy field describes by its integral curves only the trajectories of point particles that are exposed to the field (Bossavit (1998b)). We therefore conclude that covector fields are optimal descriptors as their equipotential surfaces of $\mathcal{F}$ describe the entire field while employing only minimal mathematical structure.

\subsubsection{From the mathematical descriptors to the model equations}

For simulations, we require closed sets of (geophysical) fluid equations which describe the physical phenomena. These equations can be derived by (i) describing particle displacements and forces by mathematical descriptors and (ii) by solving the balance of mass, momentum, and energy equations. The solutions of the balance equations describes then the time evolution of the fluid flow. The choice of the mathematical descriptors for displacements and forces determines the form of the equations. For instance, in describing the particle displacements by the vector field $\vec{v}$ and the forces acting upon these particles by the vector field $\vec{F}$, the fluid's velocity is represented by a vector-valued equation of the form $\frac{\partial}{\partial t} \vec{v}+\ldots \propto \vec{F}$ (see e.g. Eqn. (26)), which follows from solving the balance of momentum equation. This approach is usually followed in geophysical fluid dynamics (GFD). Alternatively, one can describe the involved forces by the above discussed covector fields $\mathcal{F}: \vec{v} \rightarrow \mathcal{F}(\vec{v}) \in \mathbb{R}$. Using a covector field $u$ to describe the fluid's velocity, the resulting equation has the form $\forall \vec{v}: \frac{\partial}{\partial t} u(\vec{v})+\ldots \propto \mathcal{F}(\vec{v})$ (see e.g. Eqn. (42)), which follows from solving a covariant master balance law. In the next sections, we will derive both formulations for the equations of (geophysical) fluid dynamics.

\section{The equations of GFD in vector-invariant form}

In this section we derive the equations of GFD in vector-invariant form as they are usually expressed in the geophysical community. In the next section, we follow the same approach for the derivation of the covariant equations, and in Sect. 5 we compare both sets of equations.

Here, we illustrate in terms of vector calculus how these equations descend from balance laws, following the method of Abraham et al. (1983). Using vectors to represent particle displacements and forces, we solve a vector-valued balance law to find the vector-valued momentum equation. The continuity and energy closure equations follow as solutions of scalar balance laws.

Let $(\mathcal{M}, \mathbf{g}, O r)$ be a compact, oriented, finite-dimensional Riemannian $n$-manifold (possibly with boundary). Let $\mu \in \Omega^{n}(\mathcal{M})$ be the Riemannian volume form on $\mathcal{M}$ and $d \mu$ the corresponding volume element. To describe the motion of the fluid in $\mathcal{M}$, let $x \in \mathcal{M}$ be the position of a fluid particle at time $t=0$. This particle traverses a well-defined trajectory given by the evolution operator $\varphi_{t}(x)=\varphi(x, t)$ and $\varphi(x, 0)=x$ (cf. Eqn. (13)). Let $\vec{u}(x, t)$ denote the velocity of the fluid particle at position $x$ at time $t . \vec{u} \in \mathcal{X}(\mathcal{M})$ is denoted as velocity field of the fluid and obeys the following relation: $\frac{d \varphi_{t}(x)}{d t}=\vec{u}\left(\varphi_{t}(x), t\right)$.

To find the governing equations for the fluid motion one usually applies three basic principles: (i) mass conservation, (ii) balance of momentum, (iii) energy conservation.

Mass conservation Let $\rho_{t}(x)=\rho(x, t)$ be the mass-density of the fluid and let, for any region $W \subset \mathcal{M}$ at time $t$, the fluid's mass be given by $m(W, t)=\int_{W} \rho_{t} d \mu$. In case of mass conservation the total mass of the fluid occupying a smooth region $W$ at $t=0$ remains unchanged after $t$, i.e. $\int_{\varphi_{t}(W)} \rho_{t} d \mu=\int_{W} \rho_{0} d \mu$. After some reformulations (cf. Abraham et al. (1983); Bauer (2013)) one finds

$$
\frac{\partial \rho}{\partial t}+\operatorname{div}(\rho \vec{u})=0
$$


describing the law of conservation of mass in differential (local) form, which is known as continuity equation.

Balance of momentum To derive the vector-valued momentum equation, we assume that a portion $W$ of the fluid is moving in $\mathcal{M} \subset \mathbb{R}^{3}$, i.e. $\mathcal{M}$ is an Euclidean space $\left(E_{3}, \mathbf{g}, O r\right)$. Let the momentum of this portion at time $t$ that occupied at time $t=0$ the region $W$ be given by $\int_{\varphi_{t}(W)} \rho \vec{u} d \mu$ with the fluid's velocity $\vec{u} \in \mathcal{X}\left(\mathbb{R}^{3}\right)$. Here, the integral is $\mathbb{R}^{3}$-valued and the integrations are performed componentwise. According to Newton's second law, the rate of change of momentum of this portion of fluid is given by the total force applied to it, i.e. the balance of momentum is given by

$$
\frac{d}{d t} \int_{\varphi_{t}(W)} \rho \vec{u} d \mu=\int_{\varphi_{t}(W)} \rho \vec{b} d \mu+\int_{\partial \varphi_{t}(W)} \widehat{\sigma} \cdot \vec{n} d a
$$

The first term on the right-hand side of (25) accounts for the total body forces acting on $W$ with $\vec{b}$ as the body force density. The second term accounts for the forces of stress, where $\widehat{\sigma}$ is the Cauchy stress tensor and where the surface element $d a$ is induced by the volume element $d \mu$ on the boundary $\partial W$ with outer unit normal $\vec{n} .{ }^{4}$

We only consider ideal (or perfect) fluids; the Cauchy stress tensor is therefore given in terms of a function $p(\vec{x}, t)$ called the pressure, i.e. $\widehat{\sigma}=-p \mathbf{I}$, with identity $\mathbf{I}$. Then, $\widehat{\sigma} \cdot \vec{n}=-p \mathbf{I} \cdot \vec{n}=-p \vec{n}$. Hence, for the last term in Eqn. (25) there follows: $\int_{\partial \varphi_{t}(W)} \widehat{\sigma} \cdot \vec{n} d a=-\int_{\partial \varphi_{t}(W)} p \cdot \vec{n} d a=$ $-\int_{\varphi_{t}(W)} \nabla p d \mu$. After some further reformulations, similar to those performed in Section 4.1 (cf. also Abraham et al. (1983); Bauer (2013)), Euler's equations for ideal fluids are given by

$$
\frac{\partial \vec{u}}{\partial t}+(\vec{u} \cdot \nabla) \vec{u}+\frac{1}{\rho} \nabla p=\vec{b}
$$

with $\nabla:=\left(\partial_{x}, \partial_{y}, \partial_{z}\right)$, which describe the balance of momentum in differential (local) form. For further calculations it will be useful to use Weber's transformation: $(\vec{u} \cdot \nabla) \vec{u}=\nabla\left(\vec{u}^{2} / 2\right)+(\nabla \times$ $\vec{u}) \times \vec{u}$ (see e.g. Pedlosky (1979)). The vector $\vec{\zeta}:=\nabla \times \vec{u}$ describes the relative vorticity of the fluid.

In case of the rotating earth, body forces such as gravitation, centripetal, and Coriolis forces act on each fluid particle. To determine these forces, one often represents the velocity field $\vec{u} \in \mathbb{R}^{3}$, that is measured in a frame $\mathcal{R}$ rotating with uniform angular velocity $\vec{\Omega}$, relative to an inertial frame (cf. Fig. 2). Then, the vector-invariant momentum equation on $\mathcal{R}$ is given by

$$
\frac{\partial \vec{u}}{\partial t}+(\vec{u} \cdot \nabla) \vec{u}+\frac{1}{\rho} \nabla p=-2 \vec{\Omega} \times \vec{u}-\vec{\Omega} \times(\vec{\Omega} \times \vec{r})-\nabla \Phi_{N}+\vec{F},
$$

(cf. Marshall and Plumb (2007); White et al. (2005)), in which the forces on the right-hand side are caused by earth rotation and gravitation and can be identified with the body force density $\vec{b}$ of Eqn. (26). With $\vec{r}$ we denote the position of the fluid particle relative to any fixed origin on the rotational axis of $\mathcal{R} ; \Phi_{N}$ is the Newtonian gravitational potential and $\vec{F}$ is any other force, which will not be further considered, i.e. $\vec{F}=0$.

The term $-2 \vec{\Omega} \times \vec{u}$, denoted as planetary vorticity vector, describes the Coriolis acceleration and will be discussed in more detail in Section 5. The centrifugal acceleration $-\vec{\Omega} \times(\vec{\Omega} \times \vec{r})=$ $\nabla\left(\frac{1}{2}|\vec{\Omega}|^{2} r_{\perp}^{2}\right)$ is directed outward normally to the rotation axis, with $r_{\perp}$ as the perpendicular

\footnotetext{
${ }^{4}$ More concretely, using Eqns. (14) and (16), Gauss' Theorem states: $\int_{W}(\operatorname{div} \vec{X}) \mu=\int_{\partial W} \mathbf{i}_{\vec{X}} \mu=\left.\int_{\partial W}\langle\vec{X}, \vec{n}\rangle \mu\right|_{\partial W}$ for $\vec{X} \in \mathcal{X}(W)$. Then, $d a$ is the surface element of the volume 2 -form $\left.\mu\right|_{\partial W} \in \Omega^{2}(\partial W)$.
} 
distance of the particle from this axis, and can be combined with the Newtonian gravity $-\nabla \Phi_{N}$ to the apparent gravity $\Phi_{A}:=\left(\Phi_{N}-\frac{1}{2}|\vec{\Omega}|^{2} r_{\perp}^{2}\right)$. There follows

$$
\frac{\partial \vec{u}}{\partial t}+\vec{\zeta} \times \vec{u}+\nabla\left(\vec{u}^{2} / 2\right)+\frac{1}{\rho} \nabla p=-2 \vec{\Omega} \times \vec{u}-\nabla \Phi_{A},
$$

where Weber's transformation has been used. Eqn. (28) agrees for $\vec{b}:=-2 \vec{\Omega} \times \vec{u}-\nabla \Phi_{A}$ with Eqn. (26).

Energy conservation With $\vec{u}, \rho, p$ there are $n+2$ scalar unknowns. We have, however, only $n+1$ equations yet. The needed further one, that closes the set of equations, can be derived using the law of conservation of energy.

Following again Abraham et al. (1983) and Bauer (2013), we assume that the total energy of the fluid can be written as a sum of kinetic and internal energy

$$
E_{\text {tot }}=E_{\text {kin }}+E_{\text {int }},
$$

where the kinetic energy of the fluid with velocity field $\vec{u}$ is given by

$$
E_{\text {kin }}=\frac{1}{2} \int_{\mathcal{M}} \rho\|\vec{u}\|^{2} d \mu \quad \text { with } \quad\|\vec{u}\|^{2}=\langle\vec{u}, \vec{u}\rangle .
$$

With $E_{\text {int }}$ we describe the internal energy of the fluid consisting of all internal energy sources such as intermolecular potentials and molecular vibrations. Despite the fact that the exact form of $E_{\text {int }}$ is unknown, the assumption of a certain behavior of $E_{\text {int }}$ allows us to derive the desired energy equations. We illustrate this procedure on the examples of incompressible and barotropic fluids.

Case 1) of incompressible flows The main assumption is that $E_{\text {int }}$ is constant. In case no energy is pumped into the system and the fluid does not perform work, i.e. $E_{\text {tot }}$ remains constant, the kinetic energy $E_{\text {kin }}$ should be constant too, according to Eqn. (29). It follows:

$$
0=\frac{d}{d t}\left(\frac{1}{2} \int_{\mathcal{M}} \rho\|\vec{u}\|^{2} d \mu\right)=\int_{\mathcal{M}}(\operatorname{div}(\vec{u})) p \mu,
$$

(cf. Abraham et al. (1983); Bauer (2013)). In order to fulfill Eqn. (32) for all conceivable fluid motions, we need either

$$
\operatorname{div}(\vec{u})=0 \quad \text { or } \quad p=0
$$

to hold. $p=0$ is possible but not further considered. If instead we require $\operatorname{div}(\vec{u})=0$, we consider the case of incompressible fluids with the boundary condition $\mathbf{i}_{\vec{u}} \mu=0$ on $\partial \mathcal{M}$.

Case 2) of barotropic (or ideal isentropic) flows The main assumption is that the internal energy $E_{\text {int }}$ (here not constant) over a region $W$ is a function of the internal energy density per unit of mass, denoted with $w$, i.e.

$$
E_{\text {int }}=\int_{W} \rho w d \mu .
$$

The energy should be balanced such that the rate of change of energy within a region $W$ equals the work done on it:

$$
\frac{d}{d t}\left(\int_{\varphi_{t}(W)} \rho \frac{1}{2}\|\vec{u}\|^{2} d \mu+\rho w d \mu\right)=-\int_{\partial \varphi_{t}(W)} p \vec{u} \cdot \vec{n} d a .
$$


Moreover, one may apply the so-called barotropic assumptions, i.e. the internal energy density $w=w(\rho) \in \Omega^{0}(\mathcal{M})$ only depends on how much the fluid is compressed. Such fluids are called ideal isentropic or barotropic. Under these assumptions, Eqn. (34) can be reformulated (cf. Abraham et al. (1983); Bauer (2013)) to

$$
0=\left(p-\rho^{2} \frac{\partial w}{\partial \rho}\right) \operatorname{div}(\vec{u}) \quad \Rightarrow \quad p=\rho^{2} \frac{\partial w}{\partial \rho},
$$

as the value for the divergence $\operatorname{div}(\vec{u})$ is not restricted. The equation on the right-hand side of (35) is called an equation of state.

Equations of GFD in vector-invariant form In sum, the vector-invariant equations of GFD in a 3-dimensional oriented Euclidean space $\left(E_{3}, \mathbf{g}, O r\right)$, rotating with uniform angular velocity $\vec{\Omega}$ relative to an inertial frame in $\mathbb{R}^{3}$, are given by

$$
\begin{array}{r}
\frac{\partial \vec{u}}{\partial t}+(\vec{\zeta}+2 \vec{\Omega}) \times \vec{u}+\frac{1}{\rho} \nabla p+\nabla\left(\vec{u}^{2} / 2\right)+\nabla \Phi_{A}=0, \\
\frac{\partial \rho}{\partial t}+\operatorname{div}(\rho \vec{u})=0,
\end{array}
$$

(with boundary condition) $\langle\vec{u}, \vec{n}\rangle=0$,

with initial conditions $\vec{u}(x, 0)=\vec{u}_{0}(x)$ and with relative vorticity $\vec{\zeta}:=\nabla \times \vec{u}$. Using the energy equations from above, this system of equations can be closed. One obtains either

1. the incompressible fluid equations assuming $\operatorname{div}(\vec{u})=0$ or

2. the barotropic fluid equations using the equation of state $p=\rho^{2} \frac{\partial w}{\partial \rho}$ with $w=w(\rho)$.

\section{The equations of GFD in covariant form}

The aim of this section is to introduce covariant equations describing the time evolution of the fluid motion on general (possibly rotating) manifolds. To derive these equations, we represent particle displacements by vectors but, in contrast to Sect. 3, we represent forces by linear maps. Covariant fluid equations valid on oriented Riemannian manifolds $(\mathcal{M}, \mathbf{g}, O r)$ follow as solutions of the covariant master balance law (37) (cf. Marsden and Hughes (1983)).

Having started our derivations in Sect. 3 from $n$-dimensional manifolds $(\mathcal{M}, \mathbf{g}, O r)$ by using scalar balance laws such as (34), which agree with the covariant master balance law (37), the continuity equation (24) and the energy equations (32) and (35) are covariant and hence valid on $\mathcal{M}$.

Since the derivation of the vector-valued momentum equation (26) requires its componentwise representation in $\mathbb{R}^{3}$, it is neither valid on general manifolds $\mathcal{M}$ nor covariant. To derive (Sect. 4.1) covariant $n$-dimensional equations for ideal (perfect) fluids, we use the covariant master balance law (37). As a second step (Sect. 4.2), we restrict our considerations to general manifolds embedded in $\mathbb{R}^{3}$. Then, we introduce to the best of our knowledge new (3-dimensional) covariant equations of GFD.

\subsection{Derivation of covariant equations for ideal fluids using the master balance law}

Similarly to Sect. (3), let $(\mathcal{M}, \mathbf{g}, O r)$, or short $\mathcal{M}$, be a compact, oriented, finite-dimensional Riemannian $n$-manifold (possibly with boundary) with volume form $\mu \in \Omega^{n}(\mathcal{M})$, volume element $d \mu$, evolution operator $\varphi_{t}(x)=\varphi(x, t)$ and $\varphi(x, 0)=x$ for $x \in \mathcal{M}, t=0$, and velocity field $\vec{u}(x, t)$. On these manifolds, the following master balance law can be formulated. 
The master balance law (Marsden and Hughes (1983)) Let $a(x, t), b(x, t)$ be scalar fields, $\vec{c}(x, t)$ be a vector field on a portion $W \subset \mathcal{M}$ of a Riemannian $n$-manifold $\mathcal{M}$. Let $\varphi_{t}(W)$ be a $C^{1}$ regular motion on $\mathcal{M}$. $a, b, \vec{c}$ satisfy the master balance law if, for any nice open set $W \subset \mathcal{M}$, the integrals that appear in the following exist, $\int_{\varphi_{t}(W)} a d \mu$ is $t$-differentiable, and

$$
\frac{d}{d t} \int_{\varphi_{t}(W)} a d \mu=\int_{\varphi_{t}(W)} b d \mu+\int_{\partial \varphi_{t}(W)}\langle\vec{c}, \vec{n}\rangle d a
$$

Analogously to Eqn. (25), the surface element $d a$ is induced by the volume element $d \mu$ on the boundary $\partial W$ with outer unit normal $\vec{n}$. This master balance law for scalars is covariant and valid on general manifolds $\mathcal{M}$ (Marsden and Hughes (1983)).

The covariant fluid equations We use the covariant master balance law (37), valid for smooth functions on $\mathcal{M}$, to derive the covariant momentum equation. Here, we exploit the fact that smooth 1-forms map from the space of smooth vector fields $\mathcal{X}(\mathcal{M})$ to the space of smooth functions $C(\mathcal{M})$. Hence, instead of considering in Eqn. (25) the vector components of $\vec{u}, \vec{b}$, and $\widehat{\sigma} \cdot \vec{n}$ (or $\nabla p$ in case of an ideal fluid), we represent the velocity, the external forces, and the pressure gradient as 1 -forms $\vec{u}^{b}(x, t), \vec{b}^{b}(x, t)$, and $\mathbf{d} p(x, t)$, respectively, which map a test vector field $\vec{w}(x) \in \mathcal{X}(\mathcal{M})$ to the space of smooth functions $C(\mathcal{M})$. The $b$ superscript indicates the unique relation between the 1-forms and the corresponding vector proxies (cf. Eqn. (19)).

On a local coordinate chart with basis $\partial_{i}:=\frac{\partial}{\partial x^{i}}$ and dual basis $d x^{i}$ with $d x^{i}\left(\partial_{j}\right)=\delta_{i j}$ for $i, j=1, \ldots, n$, the 1 -forms can be represented by $\omega=\sum_{i} \omega_{i} d x^{i}$. Without restriction of generality, we can assume that $\vec{w}(x)=\partial_{i}$ and hence $\omega\left(\partial_{i}\right)=\omega_{i}$. Then, there follows for Eq. (25)

$$
\frac{d}{d t} \int_{\varphi_{t}(W)}(\rho u)_{i} d \mu=\int_{\varphi_{t}(W)}(\rho b)_{i} d \mu-\int_{\varphi_{t}(W)}(\mathbf{d} p)_{i} d \mu \quad \forall i
$$

which balances the $i^{\text {th }}$-coefficient functions of the corresponding 1-forms and the density function $\rho$. As these functions are smooth on $\mathcal{M}$, they obey the covariant master balance law (37).

By the change-of-variable formula and the Lie derivative formula, there follows

$$
\begin{aligned}
\frac{d}{d t} \int_{\varphi_{t}(W)}(\rho u)_{i} d \mu & =\int_{W} \frac{d}{d t} \varphi_{t}^{*}\left((\rho u)_{i} \mu\right)=\int_{W} \varphi_{t}^{*} \mathcal{L}_{\vec{u}}\left((\rho u)_{i} \mu\right) \\
& =\int_{\varphi_{t}(W)}\left[\frac{\partial(\rho u)_{i}}{\partial t} \mu+\left(\mathcal{L}_{\vec{u}} \rho\right) u_{i} \mu+\rho\left(\mathcal{L}_{\vec{u}} u_{i}\right) \mu+(\rho u)_{i} \mathcal{L}_{\vec{u}} \mu\right] \\
& =\int_{\varphi_{t}(W)}\left[\frac{\partial \rho}{\partial t} u_{i}+\rho \frac{\partial u_{i}}{\partial t}+(\mathbf{d} \rho \cdot \vec{u}) u_{i}+\rho \mathcal{L}_{\vec{u}} u_{i}+\rho u_{i} \operatorname{div} \vec{u}\right] d \mu
\end{aligned}
$$

using the identities $\mathcal{L}_{\vec{u}} \mu=(\operatorname{div} \vec{u}) \mu$ and $\mathcal{L}_{\vec{u}} \rho=\mathbf{d} \rho \cdot \vec{u}$. Then, the $i^{t h}$-coefficient functions of Eqn. (38) are balanced as

$$
\left(\frac{\partial \rho}{\partial t}+(\mathbf{d} \rho \cdot \vec{u})+\rho \operatorname{div} \vec{u}\right) u_{i}+\rho \frac{\partial u_{i}}{\partial t}+\rho \mathcal{L}_{\vec{u}} u_{i}=(\rho b)_{i}-(\mathbf{d} p)_{i} .
$$

The term in brackets on the very left vanishes by conservation of mass (24), as $\mathbf{d} \rho \cdot \vec{u}+\rho \operatorname{div} \vec{u}=$ $\operatorname{div}(\rho \vec{u})$. To find a covariant expression for $\mathcal{L}_{\vec{u}} u_{i}=\sum_{j} u^{j} \frac{\partial u_{i}}{\partial x^{j}}$ with $\vec{u}=\sum_{j} u^{j} \partial_{j}$, we exploit the fact that the coordinate representations of $\mathcal{L}_{\vec{u}}$ acting on either a 0 -form or a 1 -form are related by

$$
\mathcal{L}_{\vec{u}} u_{i}=\sum_{j}[\underbrace{u^{j} \frac{\partial u_{i}}{\partial x^{j}}+u^{j} \frac{\partial u_{j}}{\partial x^{i}}}-\underbrace{u^{j} \frac{\partial u_{j}}{\partial x^{i}}}]=\left(\mathcal{L}_{\vec{u}} u\right)_{i}-\left(\mathbf{d} \frac{1}{2} u(\vec{u})\right)_{i}
$$


Summing up the coefficients of the corresponding 1-forms leads to the covariant momentum equation for ideal fluids

$$
\frac{\partial \vec{u}^{b}}{\partial t}+\mathcal{L}_{\vec{u}}\left(\vec{u}^{b}\right)-\frac{1}{2} \mathbf{d}\left(\vec{u}^{b}(\vec{u})\right)+\frac{1}{\rho} \mathbf{d} p=\vec{b}^{b}
$$

which is valid on $n$-dimensional compact Riemannian manifolds with smooth boundary $\partial \mathcal{M}$ and outward unit normal $\vec{n}$.

Remark 7 . The $n$-dimensional covariant momentum equation (42) agrees with that derived by Abraham et al. (1983). The authors generalized therein vector-invariant fluid equations by using the Riemannian lift b of Def. (19) and they performed reformulations to the Lie derivative similar to those introduced in this section.

As this will be useful later on, in particular when comparing the covariant and vectorinvariant equations in Sect. 5, we further modify the term including the Lie derivative $\mathcal{L}$ in Eqn. (42). $\mathcal{L}$ can be represented using the exterior derivative $\mathbf{d}$ and the interior product $\mathbf{i}$ (cf. Eqn. (12) (iv)). Thus, $\mathcal{L}$ applied to the 1 -form $\vec{u}^{b}$ may be written as $\mathcal{L}_{\vec{u}} \vec{u}^{b}=\mathbf{d i}_{\vec{u}}\left(\vec{u}^{b}\right)+\mathbf{i}_{\vec{u}} \mathbf{d} \vec{u}^{b}$, whereas $\mathbf{d i}_{\vec{u}}\left(\vec{u}^{b}\right)=\mathbf{d}\left(\vec{u}^{b}(\vec{u})\right.$ ). The function $\mathbf{i}_{\vec{u}} \vec{u}^{b}=\vec{u}^{b}(\vec{u})$ (cf. Corollary 3) represents twice the kinetic energy and the 2 -form $\mathbf{d} \vec{u}^{b} \in \Omega^{2}(\mathcal{M})$ the covariant relative vorticity.

\subsection{Derivation of covariant equations of GFD for the rotating earth}

In the covariant equation (42), additional forces are taken into account by the body force density 1 -form $\vec{b}^{b} \in \Omega^{1}(\mathcal{M})$. In order to determine $\vec{b}^{b}$ in case of geophysical fluid flow, we restrict the following discussion to Riemannian $k$-manifolds, with $k \leq 3$, embedded in $\mathbb{R}^{3}$ and uniformly rotating with $\vec{\Omega}$. Then, according to Eqn. (28) and by the linearity of the isomorphism $b$ (cf. Bauer (2013)), the body force density 1-form is given by $\vec{b}^{b}=-2(\vec{\Omega} \times \vec{u})^{b}-\left(\nabla \Phi_{A}\right)^{b}$. For the latter term, we use, as in Sect. 4.1, the exterior derivative $\mathbf{d}$ acting on the function $\Phi_{A} \in \Omega^{0}(\mathcal{M})$ instead of the gradient operator $\nabla$, i.e. $\left(\nabla \Phi_{A}\right)^{b}=\mathbf{d} \Phi_{A}$.

To further reformulate the covariant Coriolis acceleration term $2(\vec{\Omega} \times \vec{u})^{b}$, we first introduce a lemma of Hirani (2003) which will be rather useful here and for some subsequent proofs.

Lemma 1. (Hirani (2003)) Let $\vec{X} \in \mathcal{X}(\mathcal{M})$ be a vector field and $\alpha \in \Omega^{k}(\mathcal{M})$ a $k$-form on a smooth $n$-manifold $\mathcal{M}$. Then, the interior product can be computed with the following formula:

$$
\mathbf{i}_{\vec{X}} \alpha=(-1)^{k(n-k)} \star\left(\star \alpha \wedge \vec{X}^{b}\right) .
$$

In addition to Lemma 1 , we use a representation of the cross product in terms of differential forms by $\vec{v} \times \vec{w}=\left[\star\left(\vec{v}^{b} \wedge \vec{w}^{b}\right)\right]^{\sharp}$ for any vectors $\vec{v}, \vec{w} \in \mathbb{R}^{3}$ (cf. Abraham et al. (1983)). As $\sharp$ is the inverse of $b$, this representation allows us to express the Coriolis acceleration term as $2(\vec{\Omega} \times \vec{u})^{b}=2 \star\left(\vec{\Omega}^{b} \wedge \vec{u}^{b}\right)$. On the other hand, the right-hand side of the latter equation can be written in terms of an inner product using Lemma 1. To this end, we first apply the Hodge star $\star$ on the (3-dimensional) 1-form $\vec{\Omega}^{b}$. This results in the 2 -form $\star \vec{\Omega}^{b} \in \Omega^{2}\left(\mathbb{R}^{3}\right)$ valid for any $k$-manifold embedded in $\mathbb{R}^{3}$ and rotating with $\vec{\Omega} \in \mathbb{R}^{3}$. Then, if we associate in Eqn. (43) $\alpha$ with the 2 -form $\star \vec{\Omega}^{b}$, there follows

$$
\mathbf{i}_{\vec{u}}\left(2 \star \vec{\Omega}^{b}\right)=2(-1)^{2(3-2)} \star\left(\star \star \vec{\Omega}^{b} \wedge \vec{u}^{b}\right)=2 \star\left(\vec{\Omega}^{b} \wedge \vec{u}^{b}\right),
$$

using $\star \star \vec{\Omega}^{b}=(-1)^{k(n-k)} \vec{\Omega}^{b}=\vec{\Omega}^{b} \in \Omega^{1}\left(\mathbb{R}^{3}\right)$ (cf. Eqn. (21) (iii)). The notation $\star \vec{\Omega}^{b}$ of the 2 -form indicates that the $k$-manifold $\mathcal{M}$ is embedded in an ambient space $\mathbb{R}^{3}$. The 2 -form $2 \star \vec{\Omega}^{b}$ describes the covariant planetary vorticity. Using Eqn. (44), we establish the following theorem. 
Theorem 1. The covariant equations of GFD on a compact, oriented Riemannian k-manifold $(\mathcal{M}, \mathbf{g}$, Or $)$, with $k \leq 3$, rotating with uniform angular velocity $\vec{\Omega}$ relative to an inertial frame in $\mathbb{R}^{3}$ are given by

$$
\begin{aligned}
\frac{\partial \vec{u}^{b}}{\partial t}+\mathbf{i}_{\vec{u}}\left(\mathbf{d} \vec{u}^{b}+2 \star \vec{\Omega}^{b}\right)+\frac{1}{2} \mathbf{d}\left(\vec{u}^{b}(\vec{u})\right)+\frac{1}{\rho} \mathbf{d} p+\mathbf{d} \Phi_{A} & =0, \\
\frac{\partial \rho}{\partial t}+\star \mathbf{d} \star(\rho \vec{u})^{b} & =0,
\end{aligned}
$$

(with boundary condition on $\partial \mathcal{M}) \mathbf{i}_{\vec{u}} \mu=\langle\vec{u}, \vec{n}\rangle=0$,

with initial conditions $\vec{u}(x, 0)=\vec{u}_{0}(x)$. Using the energy closure equations, there follow either

1. the incompressible fluid equations assuming $\star \mathbf{d} \star \vec{u}^{b}=0$ or

2. the barotropic fluid equations using the equation of state $p=\rho^{2} \frac{\partial w}{\partial \rho}$ with $w=w(\rho)$.

In case $\mathcal{M}$ is an oriented Euclidean space $E_{3}$, these covariant equations are equivalent to the vector-invariant equations of GFD (36).

Remark 8. In order to obtain a purely differential geometrical representation of Eqn. (45), we use identity $\operatorname{div}(\rho \vec{u})=\star \mathbf{d} \star(\rho \vec{u})^{b}$ (see Corollary 4$)$. The set of equations would however also be covariant when using the conventional form of the continuity equation as in (24).

Proof. The covariance of Eqns. (45), hence their form invariance under changing coordinates, follows directly from the coordinate-independent definitions of differential forms and operators of differential geometry (cf. Section 2). Because Eqn. (44) changes sign with changing orientation, $\mathcal{M}$ has to be oriented (cf. Theorem 2 for an orientation independent formulation).

Next, we show that in case $\mathcal{M} \subset \mathbb{R}^{3}$ is an oriented Euclidean space, the covariant equations (45) correspond to the vector-invariant equations (36), which, in turn, immediately proves that Eqns. (45) provide a closed set of equations describing geophysical fluid flows. To show this equivalence, we first note that the covariant and the vector-invariant planetary vorticities agree according to Eqn. (44). In addition, we use the original form of the nonlinear advection term $\mathcal{L}_{\vec{u}} \vec{u}^{b}-\frac{1}{2} \mathbf{d}\left(\vec{u}^{b}(\vec{u})\right)$ as given in Eqn. (42), and we apply $\mathcal{L}_{\vec{u}} \vec{u}^{b}=\left(\nabla_{\vec{u}} \vec{u}\right)^{b}+\frac{1}{2} \mathbf{d}\left(\vec{u}^{b}(\vec{u})\right.$ ) (cf. Abraham et al. (1983)) with Riemannian connections $\nabla_{\vec{u}}$. The resulting term $\left(\nabla_{\vec{u}} \vec{u}\right)^{b}$, as well as the 1 -forms $\vec{u}^{b}$ and $\vec{b}^{b}$ can be mapped by $\sharp$ (cf. Def. (19)) to the vector proxies $\left(\nabla_{\vec{u}} \vec{u}\right), \vec{u}$ and $\vec{b}$, respectively. In addition, $\mathbf{d} p, \mathbf{d} \Phi_{A}$ are mapped by $\sharp$ to the gradients $\nabla p, \nabla \Phi_{A}$, respectively. This results in the vector-invariant momentum equation (36) because in $\mathbb{R}^{3}$ the Christoffel symbols are zero leading to $\vec{u} \cdot(\nabla \cdot \vec{u})=\nabla_{\vec{u}} \vec{u}$.

This proves that in $\mathbb{R}^{3}$, both covariant and vector-invariant momentum equations agree. Because of Corollary 4, the continuity equations (24) and (45) agree, too, and hence also the full set of covariant and vector-invariant equations of GFD.

Corollary 1. From Theorem 1 there follows the identity:

$$
\mathbf{i}_{\vec{u}}\left(\mathbf{d} \vec{u}^{b}+2 \star \vec{\Omega}^{b}\right)=((\vec{\zeta}+2 \vec{\Omega}) \times \vec{u})^{b} .
$$

Proof. This identity can be verified directly by using Corollary 2, Eqn. (72), and $\sharp$ to represent the left-hand side of (46) in Cartesian coordinates of $\mathbb{R}^{3}$. The resulting vector-valued representation agrees with the right-hand side of (46), where $\sharp$ too has been applied on (cf. Section 5 for more details). 
Remark 9. The restriction to $k$-manifolds with $k \leq 3$ for the derivation of Theorem 1 is necessary, as we describe their rotation with the 3 -dimensional vector $\vec{\Omega}$, or equivalently, with the covariant planetary vorticity 2 -form $\star \vec{\Omega}^{b}$. This restriction usually provides no limitation in practical terms. Nonetheless, Eqns. (42) are mathematically (not necessarily physically) meaningful for any dimension. Hence, in Sect. 6 we will introduce general $n$-dimensional covariant equations of GFD that are also valid on rotating $n$-manifolds.

Although the sets of covariant and vector-invariant equations of GFD are equivalent in $\mathbb{R}^{3}$, they behave differently, for instance, under coordinate transformations, under changing dimensions (cf. Eqns. (57) and (59)), and under changes in metric and orientation. We will study these different behaviors in more detail in Sections 5 and 9.

\section{Examples and Discussion of Part 1}

In this last section of Part 1, we introduce concrete coordinate representations for the covariant and vector-invariant equations. We represent them, as often done in geophysical fluid dynamics, on a rotating Cartesian coordinate frame, for which we introduce approximations required for the 2-dimensional equations. In terms of these coordinate representations, we identify similarities and differences between covariant and vector-invariant equations and we discuss advantages and disadvantages of these formulations.

\subsection{Rotating Cartesian coordinate frame on the sphere}

In order to represent the fluid equations on a rotating sphere, we choose an approach frequently used in geophysical applications (see e.g. Marshall and Plumb (2007)). Using spherical coordinates $(\lambda, \varphi, r)$, a local Cartesian coordinate frame $\mathcal{R}$ is positioned on the earth surface, with distance $a$ to the center of the earth, at latitude $\varphi$ and longitude $\lambda$ such that its $\left(x_{1}, x_{2}, x_{3}\right)$ directions point eastward, northward, and upward, respectively. The corresponding dual basis is given by $\left(d x^{1}, d x^{2}, d x^{3}\right)$ with $d x^{1}=a \cos \varphi d \lambda, d x^{2}=a d \varphi$, and $d x^{3}=d r$ (cf. Fig. 2). The rotation of the earth can be represented by the 3 -dimensional vector $\vec{\Omega}$ with angular velocity $|\vec{\Omega}|$. The components of $\vec{\Omega}$ in this local coordinates are given by $\vec{\Omega}=(0, \Omega \cos \varphi, \Omega \sin \varphi)$ with the uniform angular velocity $\Omega:=|\vec{\Omega}|$.

In $\mathcal{R} \subset \mathbb{R}^{3}$ we represents vectors by $\vec{v}=\sum_{i} v^{i} x_{i}$ and 1 -form by $\omega=\sum_{i} \omega_{i} d x^{i}$ with $d x^{i}\left(x_{j}\right)=$ $\delta_{i j}$ for $i, j=1,2,3$, and inner product $\langle\cdot, \cdot\rangle$ that follows from the Euclidean metric $\mathbf{g}$ with $g_{i j}=\delta_{i j}$. Here, $x_{i}$ are unit vectors while $x^{i}$ are the coordinate functions (cf. Sect. 2.1.1). Hence, the Riemannian lift $b$ acts on vectors like $b(\vec{v})=\sum_{i} b\left(v^{i} x_{i}\right)=\sum_{i} v_{i}^{b} d x^{i}=\vec{v}^{b}$ with coefficients $v_{i}^{b}=\sum_{j} v^{j} g_{i j}$ (cf. Bauer (2013)).

Choosing this representation, we implicitly apply (i) the spherical geopotential approximation and (ii) the shallow-atmosphere approximation. In the spherical geopotential approximation we assume that the earth's apparent geopotentials $\Phi_{A}:=\Phi_{N}-1 / 2|\vec{\Omega}|^{2} r_{\perp}^{2}$ (cf. Eqn. (28)) are spherical. This is in general not the case, but the deviation is sufficiently small. This assumption eases the representation of the vector-invariant equations in vector components significantly (White et al. (2005)).

The shallow-atmosphere approximation is based on the fact that the atmosphere is very thin as compared to the earth radius $a$, i.e. $r=a+x^{3} \approx a$. Then, the apparent gravitational potential simplifies to $\Phi_{A}^{\text {shw-atm }}=\left(\Phi_{N}-1 / 2|\vec{\Omega}|^{2} a^{2} \cos ^{2} \varphi\right)$ and the Newtonian gravitational potential can be represented by $\Phi_{N}=g x^{3}$ with gravity $g$. In addition, vertical velocity values (typically $\leq 1 \mathrm{~cm} / \mathrm{s}$ ) are much lower than the horizontal ones, which allows us to omit terms including them. 


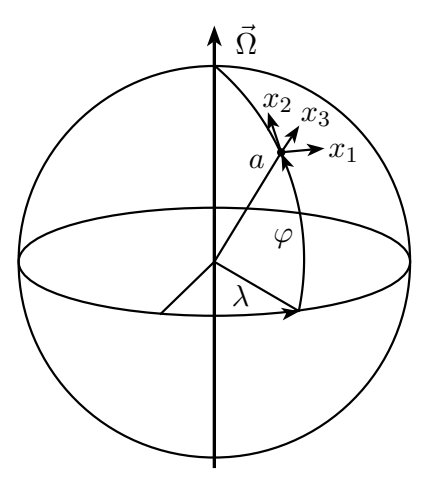

Figure 2: Representation of a rotating Cartesian coordinate frame $\mathcal{R}$, with $\left(x_{1}, x_{2}, x_{3}\right)$ of $\mathbb{R}^{3}$, at longitude $\lambda$ and latitude $\varphi$ on the surface of a sphere with radius $a$ that rotates with uniform angular velocity $|\vec{\Omega}|$.

Remark 10. The chosen Cartesian coordinate frame $\mathcal{R}$ neglects the actual earth's shape and curvature, which is a justified approximation because of the thinness of the atmosphere and ocean. There exist however more accurate representations of the equations of GFD, such as on spherical polar coordinates, which better matches the earth's actual shape (see e.g. Staniforth and Wood (2003); White et al. (2005)). The corresponding coordinate representations of the equations might be, however, more complicated.

\subsection{Representation of the covariant equations of GFD on a rotating Cartesian coordinate frame}

In this section, we provide for the covariant equations of GFD, which are given in Eqns. (45) in rather abstract form, concrete 2 - and 3-dimensional coordinate representations in $\mathcal{R}$.

\subsubsection{Representation of the 3-dimensional covariant equations}

In the coordinate frame $\mathcal{R}$ the single terms of Eqns. (45) are represented as follows. Because of Corollary 4, there is $\star \mathbf{d} \star(\rho \vec{u})^{b}=\operatorname{div}(\rho \vec{u})$ and hence $\operatorname{div}(\rho \vec{u})=\sum_{i=1}^{3} \partial_{i}\left(\rho u^{i}\right)$ with $\partial_{i}:=\frac{\partial}{\partial x^{i}}$. This already represents completely the continuity equation in $\mathcal{R}$.

In the momentum equation, the velocity proxy field is given by $\vec{u}=\sum_{i} u^{i} x_{i}$, the velocity 1-form by $u=\vec{u}^{b}=\sum_{i} u_{i} d x^{i}$ with $u_{i}:=u_{i}^{b}=u^{i} \forall i$, and the total differentials of pressure $p$, of gravitational potential $\Phi_{A}$, and of kinetic energy $\kappa=\frac{1}{2}\langle\vec{u}, \vec{u}\rangle$ (cf. Corollary 3 ) are given by the 1-forms $\mathbf{d} p=\sum_{i} \partial_{i} p d x^{i}, \mathbf{d} \Phi_{A}=\sum_{i} \partial_{i} \Phi_{A} d x^{i}$, and $\mathbf{d} \kappa=\sum_{i} \partial_{i} \kappa d x^{i}$, respectively. It remains to discuss the Coriolis and nonlinear terms.

Connection between rotation vector and rotation 2-form In Eqn. (45) the rotation of the 3-manifold is described by the 2-form $\star \vec{\Omega}^{b} \in \Omega^{2}\left(\mathbb{R}^{3}\right)$. On the other hand, a 2-form is given by $\Omega_{\text {rot }}=\sum_{1 \leq i<j \leq 3} \Omega_{i j} d x^{i} \wedge d x^{j}$. Hence, for the given representation in $\mathcal{R}$ with $\vec{\Omega}=(0, \Omega \cos \varphi, \Omega \sin \varphi)$, the relation $\Omega_{\text {rot }}=\star \vec{\Omega}^{b} \in \Omega^{2}\left(\mathbb{R}^{3}\right)$ resulting from Eqn. (44) allows us to assign values to the coefficients $\Omega_{i j}$. Therefore, we apply in succession $b$ and $\star$ on $\vec{\Omega}$, i.e.

$$
\begin{aligned}
\vec{\Omega}^{b} & =0 d x^{1}+\Omega \cos \varphi d x^{2}+\Omega \sin \varphi d x^{3} \\
\star \vec{\Omega}^{b} & =0 d x^{2} \wedge d x^{3}-\Omega \cos \varphi d x^{1} \wedge d x^{3}+\Omega \sin \varphi d x^{1} \wedge d x^{2}
\end{aligned}
$$


In $\mathbb{R}^{3}$, the 2 -form $\Omega_{\text {rot }}$ is given in local coordinates by $\Omega_{\text {rot }}=\Omega_{12} d x^{1} \wedge d x^{2}+\Omega_{23} d x^{2} \wedge d x^{3}+$ $\Omega_{13} d x^{1} \wedge d x^{3}$. Since $\Omega_{\text {rot }}=\star \vec{\Omega}^{\natural}$, we identify the following coefficients:

$$
\Omega_{12}=\Omega \sin \varphi, \quad \Omega_{13}=-\Omega \cos \varphi, \quad \Omega_{23}=0 .
$$

These coefficients describe the magnitude of angular velocity $\Omega$ of the $\left(x_{i}, x_{j}\right)$-plane in dependency of $\varphi$ (cf. Fig. 2). In case of $\varphi=0^{\circ}$, the $\left(x_{1}, x_{2}\right)$-plane does not rotate, whereas the $\left(x_{1}, x_{3}\right)$-plane does rotate with full angular velocity $-\Omega$. In case of $\varphi=90^{\circ}$, the $\left(x_{1}, x_{3}\right)$-plane does not rotate, whereas the $\left(x_{1}, x_{2}\right)$-plane rotates, but now with $+\Omega$. The $\left(x_{2}, x_{3}\right)$-plane does not rotate for any $\varphi$. The identification of the coefficients (48) allows us to formulate the following definition.

Definition 4. The 2-form $\Omega_{\text {Earth|3D }}:=\star \vec{\Omega}^{b} \in \Omega^{2}\left(\mathbb{R}^{3}\right)$ in $\mathbb{R}^{3}$ with coefficients given in (48) is called Earth-rotation 2-form. The term $2 \Omega_{\text {Earth|3D }}$ describes the covariant planetary vorticity.

This allows us to represent the Coriolis and nonlinear terms in $\mathcal{R}$. Hence, for $\Omega_{12}=\Omega \sin \varphi$, $\Omega_{13}=-\Omega \cos \varphi$, and $\Omega_{23}=0$, there follows in local coordinates by Corollary 2 and $n=3$ :

$$
\mathbf{i}_{\vec{u}} 2 \Omega_{\text {Earth } \mid 3 \mathrm{D}}=\left(2 \Omega u_{3} \cos \varphi-2 \Omega u_{2} \sin \varphi\right) d x^{1}+2 \Omega u_{1} \sin \varphi d x^{2}-2 \Omega u_{1} \cos \varphi d x^{3} .
$$

Again, by Corollary 2 and $n=3$ there follows for the nonlinear term:

$$
\mathbf{i}_{\vec{u}} \mathbf{d} u=\left(\omega_{31} u_{3}-\omega_{12} u_{2}\right) d x^{1}+\left(\omega_{12} u_{1}-\omega_{23} u_{3}\right) d x^{2}+\left(\omega_{23} u_{2}-\omega_{31} u_{1}\right) d x^{3}
$$

with coefficients $\omega_{12}:=\left(\partial_{1} u_{2}-\partial_{2} u_{1}\right), \omega_{23}:=\left(\partial_{2} u_{3}-\partial_{3} u_{2}\right)$, and $\omega_{31}:=\left(\partial_{3} u_{1}-\partial_{1} u_{3}\right)$ using property $\omega_{i j}=-\omega_{j i}$. According to Corollary 2, this coefficients determine the 3-dimensional 2-form $\mathbf{d} u=\sum_{i<j} \omega_{i j} d x^{j} \wedge d x^{j}$ that describes the covariant relative vorticity.

In sum, the terms presented here provide a complete representation of the covariant equations of GFD (45) on the rotating frame $\mathcal{R}$, which consists of a scalar continuity equation and of a covariant momentum equation based on 1-forms. Applying $\sharp$ on the latter, this covariant equation can also be represented in a more familiar form, namely as a vector-valued equation (see discussion below).

\subsubsection{Representation of the 2-dimensional covariant equations}

For certain cases it is of interest to employ 2-dimensional equations, such as the barotropic or shallow-water equations. These equations capture already major flow features of atmosphere and ocean (cf. Marshall and Plumb (2007)).

In the context of the rotating earth and under the shallow-atmosphere assumption, we represent the 2-dimensional equations on the horizontal $\left(x_{1}, x_{2}\right)$-plane and omit terms including the $x_{3}$-direction, such as the vertical velocities. Given the corresponding dual basis $\left(d x^{1}, d x^{2}\right)$, the divergence $\operatorname{div}(\rho \vec{u})$ of the continuity equation and the 1 -forms $\vec{u}^{b}, \mathbf{d} p, \mathbf{d} \Phi_{A}$, and $\mathbf{d} \kappa$ of the momentum equation of Eqns. (45) are exactly those of Section 5.2.1, but for the index $i=1,2$.

The rotation 2-form $\Omega_{\text {Earth|3D }}$ of Definition 4 reduces in two dimensions to $\Omega_{\text {Earth|2D }}:=$ $\Omega_{12} d x^{1} \wedge d x^{2}$ with coefficient $\Omega_{12}=\Omega \sin \varphi$ of Eqn. (48), whereas $\Omega_{13}=\Omega_{23}=0 . \Omega_{\text {Earth|2D }}$ is called Coriolis 2-form and describes the (horizontal) 2-dimensional covariant planetary vorticity. Then, according to Corollary 2 for $n=2$, there follows the 2-dimensional Coriolis term in local coordinates by

$$
\mathbf{i}_{\vec{u}} 2 \Omega_{\operatorname{Earth} \mid 2 \mathrm{D}}=-2 \Omega u_{2} \sin \varphi d x^{1}+2 \Omega u_{1} \sin \varphi d x^{2} .
$$

Similarly, according to Corollary 2 for $n=2$, there follows the 2-dimensional nonlinear term in local coordinates by

$$
\mathbf{i}_{\vec{u}} \mathbf{d} u=-\omega_{12} u_{2} d x^{1}+\omega_{12} u_{1} d x^{2}
$$


with coefficient $\omega_{12}:=\left(\partial_{1} u_{2}-\partial_{2} u_{1}\right)$, which determines the 2-dimensional 2-form $\mathbf{d} u=\omega_{12} d x^{1} \wedge$ $d x^{2}$, i.e. the covariant relative vorticity.

Similarly to Sect. 5.2.1, these terms provide a complete 2-dimensional representation in the horizontal $\left(x_{1}, x_{2}\right)$-plane of $\mathcal{R}$. Also here, the covariant momentum equation can be mapped by $\sharp$ to the corresponding vector-valued version.

\subsection{Representation of the vector-invariant equations of GFD on a rotating Cartesian coordinate frame}

In this section, we provide for the vector-invariant equations of GFD (36) concrete 2- and 3dimensional representations in $\mathcal{R}$. These derivations will serve us in the next section to compare the representations of vector-invariant and covariant equations.

\subsubsection{Representation of the 3-dimensional vector-invariant equations}

Here, we represent the vector-invariant equations of GFD (36) in terms of the rotating Cartesian coordinate frame $\mathcal{R}$ of Fig. 2. In $\mathcal{R}$, any vector is given by $\vec{v}=\sum_{i} v^{i} x_{i}$ and we use, as usually done, the coefficient vector $\left(v^{1}, v^{2}, v^{3}\right)$ to describe it. The Nabla-operator is given by the vector $\nabla:=\left(\frac{\partial}{\partial x^{1}}, \frac{\partial}{\partial x^{2}}, \frac{\partial}{\partial x^{3}}\right)$.

Analogously to Eqn. (45), there follows for the divergence $\operatorname{div}(\rho \vec{u})=\nabla \cdot(\rho \vec{u})=\sum_{i=1}^{3} \partial_{i}\left(\rho u^{i}\right)$, which completely represents the continuity equation (36). For the momentum equation (36), the velocity is given by $\left(u^{1}, u^{2}, u^{3}\right)$, and the gradients of the functions $p, \Phi_{A}$, and $\kappa$ are given by $\nabla p:=\left(\partial_{1} p, \partial_{2} p, \partial_{3} p\right), \nabla \Phi_{A}$, and $\nabla \kappa$, respectively. The representation of the Coriolis term that depends on $\vec{\Omega}$ is given in $\mathcal{R}$ by

$$
2 \vec{\Omega} \times \vec{u}=2\left(\begin{array}{c}
\Omega u^{3} \cos \varphi-\Omega u^{2} \sin \varphi \\
\Omega u^{1} \sin \varphi \\
-\Omega u^{1} \cos \varphi
\end{array}\right) .
$$

Given the vorticity vector $\vec{\zeta}=\left(\zeta^{1}, \zeta^{2}, \zeta^{3}\right):=(\nabla \times \vec{u})$ with $\zeta^{1}=\left(\partial_{2} u^{3}-\partial_{3} u^{2}\right), \zeta^{2}=\left(\partial_{3} u^{1}-\partial_{1} u^{3}\right)$, and $\zeta^{3}=\left(\partial_{1} u^{2}-\partial_{2} u^{1}\right)$, there follows furthermore:

$$
\vec{\zeta} \times \vec{u}=\left(\begin{array}{c}
\zeta^{2} u^{3}-\zeta^{3} u^{2} \\
\zeta^{3} u^{1}-\zeta^{1} u^{3} \\
\zeta^{1} u^{2}-\zeta^{2} u^{1}
\end{array}\right)
$$

Term (53) describes the planetary and term (54) the relative vorticity. All these terms together provide a complete 3 -dimensional representation of the vector-invariant equations of GFD (36).

\subsubsection{Representation of the 2-dimensional vector-invariant equations}

We want to present also for the vector-invariant equations (36) a 2-dimensional representation in $\mathcal{R}$, in particular on the horizontal $\left(x_{1}, x_{2}\right)$-plane. Then, vector fields are given by component vectors $\vec{v}=\left(v^{1}, v^{2}, 0\right)$ and the Nabla-operator is given by $\nabla_{h}=\left(\frac{\partial}{\partial x^{1}}, \frac{\partial}{\partial x^{2}}, 0\right)$. Similarly to the 3-dimensional case, there follow for the divergence $\operatorname{div}(\rho \vec{u})=\nabla_{h} \cdot(\rho \vec{u})=\sum_{i=1}^{2} \partial_{i}\left(\rho u^{i}\right)$ and for the gradients $\nabla_{h} p=\left(\partial_{1} p, \partial_{2} p, 0\right), \nabla_{h} \Phi_{A}$, and $\nabla_{h} \kappa$.

As the Coriolis and nonlinear terms apply the cross product, which is only defined in $\mathbb{R}^{3}$, their 2-dimensional representations require certain approximations. 
Approximations of the Coriolis and nonlinear terms The Coriolis term (53) indicates that the earth rotation influences the fluid flow in all three dimensions. This is true also for the nonlinear term (54). To find nevertheless valid 2-dimensional approximations on the horizontal $\left(x_{1}, x_{2}\right)$-plane of these terms, we assume the following (according to Marshall and Plumb (2007) good) approximations:

1. Because of the thinness of atmosphere and ocean, vertical velocities are much smaller than horizontal ones, which allows us to omit the terms including $u^{3}$ in the Coriolis term;

2. Since the $x_{3}$-component acts in opposite direction to gravity, but with negligible strength as $\Omega u^{1} \ll g$, we neglect the influence of the vertical $x_{3}$-component of the Coriolis term.

Applying these two approximations on (53), there follows the 2-dimensional approximated Coriolis term

$$
2 \vec{\Omega} \times \vec{u} \approx\left(\begin{array}{c}
-2 \Omega u^{2} \sin \varphi \\
2 \Omega u^{1} \sin \varphi \\
0
\end{array}\right)=f \vec{k} \times \vec{u},
$$

with $\vec{k}=(0,0,1)$. Usually, $f:=2 \Omega \sin \varphi$ is denoted as Coriolis parameter. The Coriolis term (55) describing the 2-dimensional planetary vorticity takes only horizontal effects of the earth rotation into account.

In case we apply these approximations to (54), namely neglecting the $x_{3}$-direction and setting $u^{3}=0$ which leads to $\zeta^{1}=\zeta^{2}=0$, there follows the 2-dimensional nonlinear term:

$$
\zeta^{3}(\vec{k} \times \vec{u})=\left(\begin{array}{c}
-\zeta^{3} u^{2} \\
\zeta^{3} u^{1} \\
0
\end{array}\right)
$$

with $\vec{k}=(0,0,1), \quad \zeta^{3}=\vec{k} \cdot \vec{\zeta}$, and $\vec{\zeta}=\nabla \times \vec{u}$. Term (56) describes the 2-dimensional relative vorticity. All these terms together provide a complete 2-dimensional representation of Eqns. (36).

\subsection{Identities between representations of covariant and vector-invariant equa- tions}

At this point, we can directly compare the representations of the covariant (Sect. 5.2) and vectorinvariant (Sect. 5.3) equations in $\mathcal{R}$. As a direct consequence of Corollary 4, the continuity equations in (45) and (36) agree in these representations.

As mentioned at the end of Sect. 5.2, the 1-forms in the covariant momentum equation can be mapped by $\sharp$ to their corresponding vector proxies. The coefficients of a 1 -form $\vec{v}^{b}=\sum_{i} v_{i} d x^{i}$ and its vector proxy $\vec{v}=\sum_{i} v^{i} x_{i}$ are related by $v_{i}=\sum_{j} v^{j} g_{i j}=v^{i}$ as $g_{i j}=\delta_{i j}$ in $\mathcal{R}$ (cf. Sect. 5.1). Hence, $\sharp$ (the inverse of $b$ ) maps the 1 -form $\vec{v}^{b}$ to the vector $\left(\vec{v}^{b}\right)^{\sharp}=\vec{v}$ with component vector $\left(v^{1}, v^{2}, v^{3}\right)$ in case of three, and $\left(v^{1}, v^{2}, 0\right)$ in case of two dimensions. Under these mappings, the representations of the covariant and vector-invariant momentum equations agree in $\mathcal{R}$ for two and three dimension, in agreement with Theorem 1.

As a consequence of this equivalence, the following identities between covariant and vectorinvariant representations of the Coriolis and nonlinear terms exist in $\mathcal{R}$ :

$$
\begin{array}{ll}
\text { in 3 dimensions: } & \mathbf{i}_{\vec{u}}\left(\mathbf{d} \vec{u}^{b}+2 \Omega_{\text {Earth } \mid 3 \mathrm{D}}\right)=((\vec{\zeta}+2 \vec{\Omega}) \times \vec{u})^{b}, \\
& \mathbf{i}_{\vec{u}}\left(\mathbf{d} \vec{u}^{b}+2 \Omega_{\text {Earth } \mid 2 \mathrm{D}}\right)=((\vec{\zeta}+f \vec{k}) \times \vec{u})^{b}, \\
\text { in 2 dimensions: } & \mathbf{i}_{\vec{u}}\left(\mathbf{d} \vec{u}^{b}+2 \Omega_{\text {Earth } \mid 2 \mathrm{D}}\right)=\left(\left(\zeta^{3}+f\right) \vec{k} \times \vec{u}\right)^{b},
\end{array}
$$


in which $\vec{\zeta}=\nabla \times \vec{u}$ denotes the full relative vorticity vector and $\zeta^{3}:=\vec{k} \cdot \vec{\zeta}$ its vertical component with $\vec{k}=(0,0,1) . \Omega_{\text {Earth|3D }}$ is the 3-, and $\Omega_{\text {Earth|2D }}$ the 2-dimensional Earth-rotation 2-form of Definition 4 with coefficients (48) or (51), respectively, resulting from $\vec{\Omega}=(0, \Omega \cos \varphi, \Omega \sin \varphi)$.

Applying $b$ or $\sharp$, identity (57) results from comparing Eqns. (53) and (54) with (49) and (50), respectively, while considering $\zeta^{1}=\omega_{23}, \zeta^{2}=\omega_{31}, \zeta^{3}=\omega_{12}$; identity (59) results from comparing Eqns. (55) and (56) with (51) and (52), respectively. Identity (58) follows directly from (57) when using the approximated Coriolis terms of (51) and (55), because for $\Omega_{\text {Earth|2D }}=\Omega_{12} d x^{1} \wedge d x^{2}$ there is $f=2 \Omega \sin \varphi=2 \Omega_{12}$ by Eqn. (48).

Remark 11. A comparison of the identities (57), (58), and (59) reveals that:

- the form of the covariant terms on the left-hand side is invariant under changes in dimension, whereas the form of the vector-invariant terms on the right-hand side changes;

- the covariant terms are well-defined on topological $n$-manifolds $\mathcal{M}$, whereas the vectorvalued terms require an oriented 3-dimensional Euclidean space $\left(E_{3}, \mathbf{g}, O r\right)$;

- the approximated Coriolis terms $\Omega_{\text {Earth } \mid 2 \mathrm{D}}$ and $f \vec{k} \times \vec{u}$ can also be used in the 3-dimensional equations. They provide good approximations and often ease calculations significantly, in particular for the vector-invariant equations.

Remark 12. Exterior calculus enables to represent the horizontal Coriolis term (51) in a 2dimensional ambient space $\mathbb{R}^{2}$. In case of vector calculus, a purely 2-dimensional representation is not possible, because the cross product only exists in 3 (and 7) dimensions (cf. Bossavit (2005) and references therein). In order to nevertheless represent the approximated 2-dimensional Coriolis term in the $\left(x_{1}, x_{2}\right)$-plane of Fig. 2 in terms of vector calculus, one requires an auxiliary 3 -dimensional vector $\vec{k}$ pointing in the $x_{3}$-direction to formulate the cross product (cf. Eqn. (55)).

Remark 13. If we compare the derivations leading either to the 2-dimensional covariant (Sect. 5.2.2) or to the vector-invariant (Sect. 5.3.2) equations, we note that the covariant ones follow naturally by the rules of exterior calculus when using 2-dimensional $k$-forms, such as $\vec{u}^{b}=u_{1} d x^{1}+u_{2} d x^{2}$ and $\Omega_{\text {Earth } \mid 2 \mathrm{D}}=\Omega_{12} d x^{1} \wedge d x^{2}$. In contrast, as the cross product requires $\mathbb{R}^{3}$, a purely 2-dimensional derivation of the 2-dimensional Coriolis term (55) is not possible, which makes it necessary to perform the above discussed approximations on term (53), i.e. omitting terms including $x_{3}$ and $u^{3}$ (cf. Sect. 5.3.2).

The 2-dimensional covariant equations also respect these approximations. This can be illustrated omitting $d x^{3}$ and $u_{3}$ in the covariant term (49), which leads to (51). The latter term is equivalent to the 2-dimensional Coriolis term (55), which indicates that both derivations lead to the same result.

Remark 14. Here, we would like to point out an important advantage of covariant equations. As these equations are based on differential forms, they are well-defined on topological manifolds, and, in contrast to vector-invariant equations, they do not require a componentwise representation in order to assure that their integrals are well-defined. By Definition 7, integrals of differential forms over chains (sums of oriented topological manifolds) assign to the chain elements real numbers (i.e. they assign degrees of freedom to the mesh elements). The latter property will allow us in upcoming work (beyond the scope of this paper) to introduce a discretization method, similar to that presented in Bauer (2013), that does not require a componentwise representation of the equations. In contrast, vector-invariant equations to be discretized have to be represented as component equations in some coordinate system (White et al. (2005)). 


\subsection{Summary of Part 1}

In this first part of the manuscript, we discussed optimal mathematical descriptors for particle displacements and force fields, and we introduced in Theorem 1 a new form of covariant equations of GFD valid on rotating $k$-dimensional manifolds $\mathcal{M}(k \leq 3)$ that are embedded in $\mathbb{R}^{3}$. These equations, using differential forms to describe forces and descending from a covariant master balance law, are independent of coordinates and dimension. These properties follow from the definitions and calculation rules of differential geometry and exterior calculus (see Sect. 2). In contrast, the vector-invariant equations of GFD (36) written in terms of vector calculus do not share these properties: for instance, there exist neither a general definition of a vector-valued integral nor a cross product on general $n$-dimensional manifolds.

In case we regard fluids moving in a 3 -dimensional Euclidean space $E_{3}$, the vector-invariant and covariant equations of GFD are equivalent (cf. Theorem 1). We illustrated this equivalence also in terms of concrete coordinate representations for both sets of equations in Sect. 5.2 and 5.3. These representations allowed us to identify similarities, but also differences. For instance, the derivations illustrated clearly that calculations in vector calculus can be rather tedious, in particular when regarding 2-dimensional equations which apply the cross product (cf. Sect. 5.3.2). This advocates the use of exterior calculus as it generalizes the derivations to standard differential geometrical methods, which are valid in any dimension.

Although both formulations agree in $E_{3}$, they behave differently under coordinate transformations, in different dimensions, and under changing metric structures. We will discuss these issues in more detail in Sect. 8 and 9. Therein, we will also discuss their conservation properties with respect to potential vorticity. Before we come to this discussion, we will first introduce formulations of the equations of GFD that do not exist at all in terms of vector calculus.

\section{Covariant equations of GFD on rotating n-dimensional mani- folds}

In this second part of the manuscript, we will introduce to the best of our knowledge new formulations of equations of GFD, namely (i) $n$-dimensional covariant equations and (ii) hierarchicallystructured $n$-dimensional covariant equations that are split into metric-free and metric-dependent parts. Both these new formulations rely essentially on the tools of differential geometry; similar formulations do not exist in term of vector calculus. In addition, we will introduce and discuss the new features which are incorporated in these new formulations.

In this section, we introduce the new $n$-dimensional covariant equations of GFD on rotating $n$ dimensional manifolds. Since the differential forms as used in the previous covariant formulations are $n$-dimensional objects by definition, the essential step is here to represent the rotation of the $n$-manifold too with a general object that is valid for $n$-dimensions. The right mathematical tool to represent the rotation of $n$-dimensional objects is a 2-form (cf. Bossavit (1998b); Hestenes (1971); Mortari (2001)):

Definition 5. The 2-form $\Omega_{\mathrm{rot}}=\sum_{1 \leq i<j \leq n} \Omega_{i j} d x^{i} \wedge d x^{j}$, in which the coefficients $\Omega_{i j} \in \mathbb{R}$ describe the angular velocities of an $n$-dimensional object rotating in the $\left(x_{i}, x_{j}\right)$-planes, is called rotation 2-form.

Remark 15. Using an orthonormal basis, the rigid body rotation in $n$ dimensions can also be represented by skew-symmetric orthogonal $(n \times n)$-matrices, which depend on $\frac{n(n-1)}{2}$ parameters (Bossavit (1998b)). This number agrees with the number of coefficients $\Omega_{i j}$ of Definition 5 that describe the angular velocities of the rotation in the $\left(x_{i}, x_{j}\right)$-planes (cf. Mortari (2001)). 
For $n=3$, there follow three parameters. This is why vectors can describe rotation only in $\mathbb{R}^{3}$. As a consequence, the necessity to restrict Theorem 1 to $k \leq 3$ descends from describing the rotation by a vector instead of a 2 -form, i.e. by using $\star \vec{\Omega}^{b} \in \Omega^{2}\left(\mathbb{R}^{3}\right)$.

To account for the fact that differential forms are more general objects than their vector proxies, and that the latter follow from the former by using the Riemannian lift $\sharp$, i.e. for any $\omega \in \Omega^{1}(\mathcal{M})$ and $\vec{w} \in \mathcal{X}(\mathcal{M})$ there is: $\omega^{\sharp}=\vec{w}\left(\Leftrightarrow \omega=\vec{w}^{b}\right)$, we change the notation with respect to Theorem 1 . The resulting set of $n$-dimensional equations is presented in the following theorem.

Theorem 2. Let the 1-form $u \in \Omega^{1}(\mathcal{M})$, and the functions $\rho, p \in \Omega^{0}(\mathcal{M})$ describe the velocity, the density and pressure, respectively, of an ideal fluid. Then, the covariant Euler's equations of GFD on a compact, with $\Omega_{\text {rot }}$ uniformly rotating Riemannian $n$-manifold $(\mathcal{M}, \mathbf{g})$ with smooth boundary $\partial \mathcal{M}$ and outward unit normal $\vec{n}$ are given by

$$
\begin{aligned}
\frac{\partial u}{\partial t}+\mathbf{i}_{u^{\sharp}}\left(\mathbf{d} u+2 \Omega_{\mathrm{rot}}\right)+\frac{1}{2} \mathbf{d}\left(u\left(u^{\sharp}\right)\right)+\frac{1}{\rho} \mathbf{d} p+\mathbf{d} \Phi_{A^{n}} & =0, \\
\frac{\partial(\star \rho)}{\partial t}+\mathbf{d} \star(\rho u) & =0, \\
\mathbf{i}_{u^{\sharp}} \mu=\langle\vec{u}, \vec{n}\rangle d a & =0 \quad(\text { on } \partial \mathcal{M}),
\end{aligned}
$$

with $u^{\sharp}=\vec{u}$ and initial conditions $\vec{u}(x, 0)=\vec{u}_{0}(x)$. By the energy closure equations, there follows either

1. the incompressible fluid equations assuming $\star \mathbf{d} \star u=0$ or

2. the barotropic fluid equations using the equation of state $p=\rho^{2} \frac{\partial w}{\partial \rho}$ with $w=w(\rho)$.

The generalized apparent gravity is described by the potential $\Phi_{A^{n}}:=\left(\Phi_{N}-\sum_{1 \leq i<j \leq n} \frac{1}{2} \Omega_{i j}^{2} r_{i j}^{2}\right)$ with $r_{i j}$ the perpendicular distance of a particle from the fixed point of the rotating $\left(x_{i}, x_{j}\right)$-plane with angular velocity $\Omega_{i j}$.

Proof. As a direct consequence of Eqn. (42), which is valid for $n$ dimensions (cf. also Abraham et al. (1983)), it is sufficient to study only those terms in (60) that occur because of the $n$-dimensional rotation; the remaining terms including the energy closure equations (cf. Theorem 1) and the boundary condition (cf. footnote 4) stay valid for any dimension $n$. Thus, we study in the following the nonlinear Coriolis term $\mathbf{i}_{u^{\sharp}}\left(\mathbf{d} u+2 \Omega_{\text {rot }}\right)$, the apparent gravity term $\mathbf{d} \Phi_{A}$, and the continuity equation.

By linearity of the interior product, the nonlinear Coriolis term can be written as $\mathbf{i}_{u^{\sharp}} \mathbf{d} u+$ $\mathbf{i}_{u^{\sharp}} 2 \Omega_{\text {rot }}$. Adding to the first term in the latter equation twice the kinetic energy gives the Lie derivative of $u$ along $\vec{u}$, i.e. $\mathcal{L}_{u^{\sharp}} u=\mathbf{i}_{u^{\sharp}} \mathbf{d} u+\mathbf{d i}_{u^{\sharp}} u$ (cf. end of Sect. (4.1)), which is well-defined in $n$ dimensions according to Eqn. (42). It thus remains to study $\mathbf{i}_{u^{\sharp}} 2 \Omega_{\text {rot }}$.

As described in Definition 5 and in literature (Bossavit (1998b); Hestenes (1971); Mortari (2001)), the rigid body rotation in $n$ dimensions can be described by a 2 -form given in local coordinates by $\Omega_{\text {rot }}=\sum_{i<j} \Omega_{i j} d x^{i} \wedge d x^{j}$ with coefficients $\Omega_{i j}$ for $1 \leq i<j \leq n$. From a mathematical point of view, this representation agrees with the 2 -form $\mathbf{d} u=\sum_{i<j} \omega_{i j} d x^{i} \wedge d x^{j}$ that describes an $n$-dimensional rotation of the velocity field with coefficients $\omega_{i j}=(-1)^{(i+j-1)}\left(\partial_{i} u_{j}-\partial_{j} u_{i}\right)$. Consequently, the acceleration of the velocity $u$ caused by the rigid body rotation can be represented - similarly to $\mathbf{i}_{u^{\sharp}} \mathbf{d} u$ for the vorticity $\mathbf{d} u$ - with the interior product by $\mathbf{i}_{u^{\sharp}} 2 \Omega_{\text {rot }}$. As shown in Corollary 2 and Eqn. (72), the local representations of both the latter terms agree too. Thus, the term $\mathbf{i}_{u^{\sharp}}\left(\mathbf{d} u+2 \Omega_{\text {rot }}\right)$ is well-defined in $n$ dimensions. 
Next, we study the generalized apparent gravity potential $\Phi_{A^{n}}$. By linearity of $\mathbf{d}$, the gradient of this potential $\mathbf{d} \Phi_{A^{n}}$ consists of the gradient of the Newton potential $\mathbf{d} \Phi_{N}$, which is welldefined in $n$ dimensions as a consequence of the identification $\vec{b}^{b}=\mathbf{d} \Phi_{N}$ in Eqn. (42), and of the gradient of a sum of potentials $\mathbf{d}\left(\sum_{1 \leq i<j \leq n}-\frac{1}{2} \Omega_{i j}^{2} r_{i j}^{2}\right)$. To understand what the latter potentials describe, let us first investigate the 3 -dimensional case. The term $-\nabla\left(\frac{1}{2}|\vec{\Omega}|^{2} r_{\perp}^{2}\right)$ of Eqn. (28) reveals that the centrifugal force can be represented as the gradient of a centrifugal potential. The centrifugal force grows linearly with $r_{\perp}$, the perpendicular distance from the rotation axis, and quadratically with the angular velocity $|\vec{\Omega}|$. The minus sign indicates that the centrifugal force is opposite to the centripetal force and to the rotation axis. Similarly, such potentials can be formulated for every rotating $\left(x_{i}, x_{j}\right)$-plane with angular velocity $\Omega_{i j}$ and distance $r_{i j}$ from the plane's rotation axis (i.e. the fixed point of the $\left(x_{i}, x_{j}\right)$-plane under rotation). The gradient $\mathbf{d}\left(\frac{1}{2} \Omega_{i j}^{2} r_{i j}^{2}\right)$ describes the centrifugal force caused by this rotation. By linearity of the gradient operator acting on functions, the contributions of all rotating $\left(x_{i}, x_{j}\right)$-planes can be added to the sum of potentials as given by $\Phi_{A^{n}}$. The gradient of the latter describes then the total centrifugal force that is induced by the $n$-dimensional rotation. For a rotating sphere in $\mathbb{R}^{3}$, the calculation in Eqn. (77) shows that this apparent gravity potential is well-defined.

Finally, when applying $\star$ on both sides of the continuity equation (60), the conventional formulation of Eqn. (24) follows as (i) $\star \star \rho=\rho$ and (ii) $\star \mathbf{d} \star(\rho u)=\operatorname{div}(\rho \vec{u})$. As $\rho$ is a 0 -form, (i) follows from the identity $\star \star \rho=(-1)^{0(n-0)} \rho$ (cf. proof to Corollary 2). The fact that identity (ii) holds for any $n$ is shown in Corollary 4. Hence, the continuity equation (60) is well-defined for any dimension $n$. Moreover, it is independent of the choice of an orientation on $\mathcal{M}$, analogously to Eqn. (24) (cf. Sect. 9.2).

The fact that Theorem 1 requires an oriented manifold follows as the term $2 \star \vec{\Omega}^{b}$, describing the manifold's rotation, depends on orientation, since $\star$ does (cf. Sect. 7). In contrast, by the orientation independent definition of $\Omega_{\text {rot }}$, the manifold $\mathcal{M}$ of Theorem 2 need not be oriented.

We present in the following Corollaries $n$-dimensional local representations of the single terms of Eqns. (60). Gradients of functions $f \in \Omega^{0}(\mathcal{M})$ are given by $\mathbf{d} f=\sum_{i=1}^{n} \partial_{i} f d x^{i}$ with $\partial_{i}:=\frac{\partial}{\partial x^{i}}$. These representations have already been used in Section 5.1 to compare the covariant and vector-invariant equations in two and three dimensions.

Corollary 2. Let $u \in \Omega^{1}(\mathcal{M})$ be a 1-form, the relative vorticity $\mathbf{d} u$ the corresponding 2-form, and $\vec{u} \in \mathcal{X}(\mathcal{M})$ a vector field on a Riemannian n-manifold $(\mathcal{M}, \mathbf{g})$ on which the identification $u^{\sharp}=\vec{u}$ holds. Then, the following identity holds:

$$
\mathbf{i}_{\vec{u}} \mathbf{d} u=\sum_{1 \leq i<j \leq n}\left(\omega_{i j} u_{i} d x^{j}-\omega_{i j} u_{j} d x^{i}\right)
$$

and provides an $n$-dimensional local representation in the coordinates $d x^{i}, i=1, \ldots, n$, with $\mathbf{d} u=\sum_{1 \leq i<j \leq n} \omega_{i j} d x^{i} \wedge d x^{j}$ and $\omega_{i j}=(-1)^{(i+j-1)}\left(\partial_{i} u_{j}-\partial_{j} u_{i}\right)$.

Proof. In local coordinates we can represent the velocity by $u=\sum_{i=1}^{n} u_{i} d x^{i}$, which leads to $\mathbf{d} u=\sum_{1 \leq i<j \leq n} \omega_{i j} d x^{i} \wedge d x^{j}$ with $\omega_{i j}:=(-1)^{(i+j-1)}\left(\partial_{i} u_{j}-\partial_{j} u_{i}\right)$ according to the definition of d in Sect. 2.1.2. We apply Lemma 1 to represent the left-hand side of Eqn. (61). Then,

$$
\mathbf{i}_{\vec{u}} \mathbf{d} u=\star\left((\star \mathbf{d} u) \wedge \vec{u}^{b}\right)=\sum_{1 \leq i<j \leq n} \sum_{k=1}^{n} \omega_{i j} u_{k} \star\left(\star\left(d x^{i} \wedge d x^{j}\right) \wedge d x^{k}\right) .
$$

Before we can further reformulate the right-hand side of (62), we first show how the Hodge star operator acts on local coordinates $\left(d x^{i}, i=1, \ldots, n\right)$. Recall that for any $k$-forms $\alpha, \beta \in$ 
$\Omega^{k}(\mathcal{M})$ there is (i) $\alpha \wedge \star \beta=\beta \wedge \star \alpha=\langle\alpha, \beta\rangle \mu$ and (ii) $\star \star \alpha=(-1)^{k(n-k)} \alpha$ (cf. Eqn. (21)). Given the volume form $\mu=d x^{1} \wedge \ldots \wedge d x^{n}$, the Hodge star $\star$ maps the 1 -forms $d x^{i}$ for all $i=1, \ldots, n$, to the $(n-1)$-forms $\star d x^{i}$ as:

$$
\begin{aligned}
\star d x^{i} & =(-1)^{(i-1)} d x^{1} \wedge \ldots \wedge \widehat{d x^{i}} \ldots \wedge d x^{n}, \text { while } \\
\star \star d x^{i} & =(-1)^{1(n-1)} d x^{i} .
\end{aligned}
$$

The notation $\widehat{ }$ over the $i^{t h}$-entry means that it is omitted. Eqn. (63) follows from $\mu=d x^{i} \wedge \star d x^{i}=$ $d x^{i} \wedge\left((-1)^{(i-1)} d x^{1} \wedge \ldots \wedge \widehat{d x^{i}} \ldots \wedge d x^{n}\right)=(-1)^{2(i-1)} \mu=+\mu \forall i=1, \ldots, n$, where the second factor $(-1)^{(i-1)}$ arises from reordering $d x^{i}$ to its $i^{\text {th }}$ position. To show Eqn. (64), we regard the $(n-1)$-form $\alpha:=\star d x^{i}$ of (63) and calculate $c \mu=\alpha \wedge \star \alpha=\star d x^{i} \wedge \star \star d x^{i}$, applying $\star \star d x^{i}=c d x^{i}$ with some constant $c$. Using in addition the representation of Eqn. (63), there follows $c \mu=\left((-1)^{(i-1)} d x^{1} \wedge \ldots \wedge \widehat{d x^{i}} \ldots \wedge d x^{n}\right) \wedge d x^{i}=(-1)^{(n-i)+(i-1)} \mu$ and thus $c=(-1)^{(n-1)}$, in which the factor $(-1)^{(n-i)}$ follows by reordering $d x^{i}$, now from the right, to its $i^{\text {th }}$-position. For the 1-forms $d x^{i}$, Eqn. (64) thus agrees with Condition (ii). Furthermore, $\star$ maps the 2 -forms $d x^{i} \wedge d x^{j}$ for all $1 \leq i<j \leq n$ to the $(n-2)$-forms

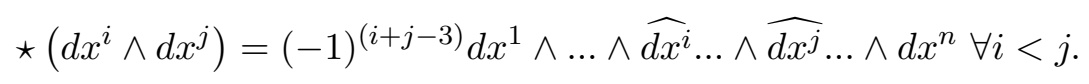

Similarly to Eqn. (63) this can be seen by calculating $\mu=d x^{i} \wedge d x^{j} \wedge \star\left(d x^{i} \wedge d x^{j}\right)$. Using Eqn. (65) and reordering provides the factor $(-1)^{(i+j-3)}$. This factor follows because moving $d x^{i}$ from position 1 to the $i^{\text {th }}$-position gives the sign $(-1)^{(i-1)}$, while permuting $d x^{j}$ from position 2 to the $j^{\text {th }}$-position gives $(-1)^{(j-2)} .^{5}$

With this, we are ready to represent the right-hand side of Eqn. (62) for all $1 \leq i<j \leq n$ and $k=1, \ldots, n$ by:

$$
\begin{aligned}
\star\left(\star\left(d x^{i} \wedge d x^{j}\right) \wedge d x^{k}\right)= & \star\left((-1)^{(i+j-3)}\left(d x^{1} \wedge \ldots \wedge \widehat{d x^{i}} \ldots \wedge \widehat{d x^{j}} \ldots \wedge d x^{n}\right) \wedge d x^{k}\right) \\
= & (-1)^{(i+j-3)+(n-j)} \delta_{j k} \star\left(d x^{1} \wedge \ldots \wedge \widehat{d x^{i}} \ldots \wedge d x^{n}\right) \\
& +(-1)^{(i+j-3)+(n-(i-1))} \delta_{i k} \star\left(d x^{1} \wedge \ldots \wedge \widehat{d x^{j}} \ldots \wedge d x^{n}\right) \\
= & (-1)^{(i+j-3)+(n-j)+(n-i)} \delta_{j k} d x^{i} \\
& +(-1)^{(i+j-3)+(n-(i-1))+(n-j)} \delta_{i k} d x^{j} \\
= & -\delta_{j k} d x^{i}+\delta_{i k} d x^{j} \quad \forall k, i<j .
\end{aligned}
$$

The factor $(-1)^{(n-j)} \delta_{j k}$ in (67) comes from reordering $d x^{k}$ from the right to position $j$, whereas $(-1)^{(n-(i-1))} \delta_{i k}$ in (68) arises from reordering $d x^{k}$ from the right to position $i$ while position $j$ is missing. The signs in Eqn. (69), $(-1)^{2 n-3}=-1 \forall n$, and in Eqn. (70), $(-1)^{2 n-2}=1 \forall n$, are independent of the indices. The local representation in Eqn. (61) follows when Eqn. (71) is used in Eqn. (62).

Remark 16. The representation of the rotation 2-form $\Omega_{\mathrm{rot}}=\sum_{i<j} \Omega_{i j} d x^{i} \wedge d x^{j}$ with coefficients $\Omega_{i j}$ describing the angular velocities of an $n$-dimensional object rotating in the $\left(x_{i}, x_{j}\right)$-planes is similar to the $n$-dimensional representation (61) of the relative vorticity 2 -form $\mathbf{d} u$ of Corollary 2 . This latter corollary is thus valid for $\Omega_{\mathrm{rot}}$ too and, moreover, as the interior product $\mathbf{i}_{u^{\sharp}}$ is linear, it follows:

$$
\mathbf{i}_{\vec{u}} \mathbf{d}(u+2 \Omega)=\sum_{1 \leq i<j \leq n}\left(\omega_{i j}+2 \Omega_{i j}\right)\left(u_{i} d x^{j}-u_{j} d x^{i}\right) .
$$

\footnotetext{
${ }^{5}$ A permutation of $d x^{k}$, for $i<j<k$, from position 3 to the $k^{t h}$-position gives the factor $(-1)^{(k-3)}$ leading to the term $\star\left(d x^{i} \wedge d x^{j} \wedge d x^{k}\right)=(-1)^{(i-1)+(j-2)+(k-3)} d x^{1} \wedge \ldots \wedge \widehat{d x^{i}} \ldots \wedge \widehat{d x^{j}} \ldots \wedge \widehat{d x^{k}} \ldots \wedge d x^{n}$, and so forth.
} 
Corollary 3. Let $u \in \Omega^{1}(\mathcal{M})$ be a 1-form and $\vec{u} \in \mathcal{X}(\mathcal{M})$ a vector field on a Riemannian $n$-manifold $(\mathcal{M}, \mathbf{g})$, on which the identification $u^{\sharp}=\vec{u}$ holds. Then, the following identities hold:

$$
\mathbf{i}_{\vec{u}} u=u(\vec{u})=\langle\vec{u}, \vec{u}\rangle,
$$

where $\kappa:=\frac{1}{2} \mathbf{i} \vec{u} u$, the kinetic energy, is a smooth function on $\mathcal{M}$.

Proof. First, we apply Lemma 1 to represent the left term in (73) while representing the 1-form $u=\sum_{i=1}^{n} u_{i} d x^{i}$ and the vector $\vec{u}=\sum_{i=1}^{n} u_{i} \partial_{i}$ in local coordinates. Then,

$$
\mathbf{i}_{\vec{u}} u=(-1)^{1(n-1)} \star\left((\star u) \wedge \vec{u}^{b}\right)=(-1)^{1(n-1)} \sum_{i, j=1}^{n} u_{i} u_{j} \underbrace{\star\left(\left(\star d x^{i}\right) \wedge d x^{j}\right)}_{=(-1)^{1(n-1)} \delta_{i j}}=\sum_{i=1}^{n} u_{i}^{2} .
$$

To reformulate $\star\left(\left(\star d x^{i}\right) \wedge d x^{j}\right)$, we insert representation (63) and then move $d x^{j}$ from the right to position $i$ to obtain the volume form $\mu$, i.e. $(-1)^{(i-1)} \star\left(\left(d x^{1} \wedge \ldots \wedge \widehat{d x^{i}} \wedge \ldots \wedge d x^{n}\right) \wedge d x^{j}\right)=$ $(-1)^{(i-1)+(n-i)} \delta_{i j} \star \mu=(-1)^{(n-1)} \delta_{i j}$, as $\mu$ is only non-zero in case $i=j$ and as $\star \mu=1$ according to Def. (21). The rightmost equality in (74) follows as $(-1)^{2(n-1)}=1 \forall n$.

The middle term in Eqn. (73) corresponds to the mapping $u: \vec{u} \mapsto u(\vec{u})=\sum_{i, j=1}^{n} u_{i} u_{j} d x^{i}\left(\partial_{j}\right)=$ $\sum_{i} u_{i}^{2}$, in which the latter identity follows from $d x^{i}\left(\partial_{j}\right)=\delta_{i j}$ for a basis and its dual. This middle term can also be represented by the Riemannian lift $b: \vec{u} \mapsto \vec{u}^{b}=\langle\vec{u}, \cdot\rangle$ of Eqn. (20) leading to $u(\vec{u})=\vec{u}^{b}(\vec{u})=\langle\vec{u}, \vec{u}\rangle=\sum_{i=1}^{n} u_{i}^{2}$, where the sum results from the definition of an inner product by Eqn. (17). Thus all terms in Eqn. (73) agree. As the inner product and the Riemannian lift are smooth operators on $\mathcal{M}, \kappa \in \Omega^{0}(\mathcal{M})$ is a smooth function on $\mathcal{M}$.

Finally, we establish in the following corollary a representation of the divergence operator in terms of operators of differential geometry.

Corollary 4. Let $\rho \in \Omega^{0}(\mathcal{M})$ be a function, $u \in \Omega^{1}(\mathcal{M})$ a 1-form and $\vec{u} \in \mathcal{X}(\mathcal{M})$ a vector field on a Riemannian n-manifold $(\mathcal{M}, \mathbf{g})$, on which the identification $u^{\sharp}=\vec{u}$ holds. Then, the following identity holds:

$$
\star \mathbf{d} \star(\rho u)=\operatorname{div}(\rho \vec{u}) .
$$

Proof. We represent the 1-form $\rho u=\sum_{i=1}^{n} \rho u_{i} d x^{i}$ with density $\rho \in \Omega^{0}(\mathcal{M})$ and the vector $\vec{u}=\sum_{i=1}^{n} u_{i} \partial_{i}$ in local coordinates and evaluate the left-hand side of Eqn. (75). Applying $\star$ on $\rho u$ gives $\sum_{i=1}^{n} \rho u_{i} \star d x^{i}$. Using the representation (63) and by the definition of $\mathbf{d}$ it follows:

$$
\star \mathbf{d} \star(\rho u)=(-1)^{(i-1)} \sum_{i, j=1}^{n} \partial_{j}\left(\rho u_{i}\right) \underbrace{\star\left(d x^{j} \wedge d x^{1} \ldots \wedge \widehat{d x^{i}} \ldots \wedge d x^{n}\right)}_{=(-1)^{(i-1)} \delta_{i j} \star \mu}=\sum_{i=1}^{n} \partial_{i}\left(\rho u_{i}\right),
$$

with $\star \mu=1$. The factor $(-1)^{(i-1)}$ in front of $\delta_{i j}$ results from reordering $d x^{j}$ to position $i$ to obtain the volume form $\mu$, which is only non-zero in case $i=j$. This leads to a positive sign of the sum for all $i$ as $(-1)^{2(i-1)}=1 \forall i$. The rightmost sum in Eqn. (76) agrees with the definition of the divergence of a vector, i.e. with $\operatorname{div}(\rho \vec{u})$.

\subsection{Representation of the covariant equations of GFD including the general- ized apparent gravity potential}

To represent, similarly to Sect. 5, the covariant equations of GFD of Theorem 2 on a rotating Cartesian coordinate frame of Fig. 2 in $\mathbb{R}^{3}$, we require only a representation of the generalized apparent gravity potential $\Phi_{A^{n}}=\left(\Phi_{N}-\sum_{1 \leq i<j \leq n} \frac{1}{2} \Omega_{i j}^{2} r_{i j}^{2}\right)$ with coefficients (48), because 
Corollaries 2, 3, and 4 provide corresponding 3-dimensional representations for the remaining terms.

As for both the $\left(x_{1}, x_{2}\right)$ - and the $\left(x_{1}, x_{3}\right)$-planes, the distance from the rotation axis $\vec{\Omega}$ is given by $r_{12}=r_{13}=a \cos \varphi$ with earth radius $a$ (see Fig. 2), there follows:

$$
\Phi_{A^{n}}=\left(\Phi_{N}-\frac{1}{2}\left(\Omega^{2} \cos ^{2} \varphi+\Omega^{2} \sin ^{2} \varphi\right) a^{2} \cos ^{2} \varphi\right)=\left(\Phi_{N}-\frac{1}{2} \Omega^{2} a^{2} \cos ^{2} \varphi\right),
$$

which agrees with the definition in Eqn. (28). This provides a complete coordinate representation of Eqns. (60) and, moreover, indicates that the generalized apparent gravity potential is welldefined.

\section{Hierarchically-structured (split) covariant equations of GFD}

The covariant fluid equations given in Theorem 2 are mathematically well-defined on rotating $n$ dimensional manifolds. Yet these equations do not precisely describe the geometrical properties of the fluid flow and the involved prognostic variables. Therefore, we introduce here a new structuring of the equations of GFD, which is based on equations (60) but takes also geometrical properties, such as the physical dimensions of the fluid's quantities of interest (e.g. density, mass-flux, vorticity), into account. Then, we explain how this new form better fits the physical properties of the fluid's quantities of interest and the fluid flow in general.

\subsection{Additionally required mathematical concepts and structures}

Before we come to this new formulation, we introduce some additional definitions and operators used in differential geometry. Some of them, such as the orientation of a manifold, have already been implicitly used above. Others, such as chains (i.e. weighted sums of manifolds) as well as the notion of straight and twisted differential forms and Hodge star operators, have not been required yet, but will be essential later on.

Orientation, inner and outer oriented manifolds Given the set of bases $\mathfrak{B}(V)$ of a real vector space $V$, two bases $\left(v_{1}, \ldots, v_{n}\right)$ and $\left(w_{1}, \ldots, w_{n}\right)$ of $\mathfrak{B}(V)$ have the same orientation, $\left(v_{1}, \ldots, v_{n}\right) \sim\left(w_{1}, \ldots, w_{n}\right)$, if one basis follows from the other by an automorphism $f: V \rightarrow$ $V, f\left(v_{i}\right)=w_{i}, i=1, \ldots n$, with positive determinant det $f>0$. Two bases with negative determinant $\operatorname{det} f<0$ have opposite orientation. The equivalence relation of having same orientation forms exactly two equivalence classes of bases on $\mathfrak{B}(V)$. These equivalence classes are called orientation of $V$. An oriented vector space is a pair $(V, O r)$ consisting of a real vector space with one of the two orientations. By convention, points (0-dimensional vector spaces) can be oriented by \pm 1 (Jänich (2001)).

Similarly, on orientable manifolds $\mathcal{M}$ (i.e. a volume form exists), two volume forms $\mu_{1}$ and $\mu_{2}$ are called equivalent, if there is an $f \in C^{\infty}(\mathcal{M})$ with $f(p)>0 \forall p \in \mathcal{M}$ such that $\mu_{1}=f \mu_{2}$. An orientation of $\mathcal{M}$ is an equivalence class $[\mu]$ of volume forms on $\mathcal{M}$. An oriented manifold $(\mathcal{M}, O r)$ is an orientable manifold $\mathcal{M}$ together with one of the two possible orientations (Abraham et al. (1983)).

We distinguish between inner and outer orientated manifolds. Let us assume a decomposition of an ambient space $V$ into two complementary subspaces $U$ and $W$, i.e. $V=U+W$. Then, an inner orientation of, say, $U$ may be assigned (e.g. by a pointing direction of a vector or a rotation direction within an area) without orienting neither its complementary space $W$ nor the ambient space $V$. In contrast, an outer orientation of a subspace, say, $V$ is given by the orientation of one of its complements $U$ or $W$. This concept of outer orientation allows us to 
define, for instance, the notion of a flux through a surface: an inner-oriented line, say, $U$ defines a crossing direction through a surface $W$ and thus outer orients the latter. In case the ambient space $V$ is oriented too, the outer orientation of $W$ also determines the inner orientation of $U$ (cf. Bossavit (1998a,c, 2005) for more details).

Straight and twisted $k$-chains The structure of orientation on topological manifolds enables to introduce chains as weighted sums of oriented manifolds and as elements of an exterior algebra.

Definition 6 (Bossavit (2005)). A $k$-chain is a finite family $\mathcal{M}=\left\{\mathcal{M}_{i}, i=1, \ldots k\right\}$ of oriented connected $k$-manifolds - denoted with components of the chain - each weighted with $\mu^{i}$ that belongs to some ring (here $\mathbb{R}$ ). We denote the chain by the formal sum: $\sum_{i} \mu^{i} \mathcal{M}_{i}:=\mu^{1} \mathcal{M}_{1}+$ $\cdots+\mu^{k} \mathcal{M}_{k}$.

Treating chains as whole objects and using formal sums allow us to treat chains according to the rules of (exterior) algebra, i.e. the sum of two chains, given by $\sum_{i} \mu^{i} \mathcal{M}_{i}+\sum_{j} \nu^{j} \mathcal{N}_{j}$, is a new chain consisting of components of the two families $\mathcal{M}$ and $\mathcal{N}$. In this context, one uses the following conventions: (i) $\mu \mathcal{M}^{\prime}$ equals $-\mu \mathcal{M}$ in case $\mathcal{M}^{\prime}$ is the same manifold as $\mathcal{M}$ with opposite orientation; (ii) in case all weights are zero, the null chain, denoted with 0 , follows.

To allow such algebraic calculations, the chains require to be consistently oriented. The chains inherit the orientation by the component manifolds. We denote a chain a straight $k$-chain if it consists of inner oriented component manifolds and twisted if it consists of outer oriented ones. Straight and twisted chains are not added or mixed in any sense.

Straight and twisted differential forms ( $k$-cochains) On oriented topological manifolds, we define, alternatively to Definition 3, differential forms as dual objects acting on chains.

Definition 7 (Bossavit (2005)). A straight (resp. twisted) differential form of degree $k$, called $k$-form, is a real valued map $\omega$ over the space of straight (resp. twisted) $k$-chains, linear with respect to chain addition, and continuous in the sense of the topology defined on the topological space.

We call $\Omega^{k}(\mathcal{M})$ and $\widetilde{\Omega}^{k}(\mathcal{M})$ the space of straight and twisted $k$-forms, respectively, and $\mathcal{C}_{k}(\mathcal{M})$ and $\tilde{\mathcal{C}}_{k}(\mathcal{M})$ the space of straight and twisted $k$-chains, respectively. $\mathcal{C}_{k}(\mathcal{M})$ and $\Omega^{k}(\mathcal{M})$ (resp. $\tilde{\mathcal{C}}_{k}(\mathcal{M})$ and $\widetilde{\Omega}^{k}(\mathcal{M})$ ) are in duality via the bilinear bicontinuous map: $<,>: \mathcal{C}_{k}(\mathcal{M}) \times$ $\Omega^{k}(\mathcal{M}) \rightarrow \mathbb{R} ;<c, \omega>\mapsto \int_{c} \omega, \forall c \in \mathcal{C}_{k}, \forall \omega \in \Omega^{k}(\mathcal{M})$. This product is nondegenerate, that is $\left.<c^{\prime}, \omega\right\rangle=0 \forall c^{\prime}$ implies $\omega=0$ and $\left\langle c, \omega^{\prime}\right\rangle=0 \forall \omega^{\prime}$ implies $c=0$ (Bossavit (2005)).

Remark 17. The differential forms of Definition 7 agree with those of Definition 3. In the latter case, the sign of the value resulting from the mapping of $k$ linearly independent vectors at point $p \in \mathcal{M}$ to $\mathbb{R}$ by the $k$-form $\omega \in \Omega^{k}(\mathcal{M})$ depends on the ordering of these $k$ vectors. As one can assign to such ordering of vectors a consistent orientation of a $k$-manifold, $k$-forms of Definition 7 , as real valued maps over the space of such (inner or outer) oriented manifolds, agree with those of Definition 3.

The integral of a differential form $\omega$ depends on the inner orientation of the manifold (or submanifold) $\mathcal{M}$ over which $\omega$ is integrated. In case of opposite inner orientation of $\mathcal{M}$, denoted with $-\mathcal{M}$, it follows $\int_{-\mathcal{M}} \omega=-\int_{\mathcal{M}} \omega$. In case it is not induced by an outer orientation, the inner orientation of a (sub)manifold is independent of the orientation of the ambient space.

Twisted differential forms and the twisted Hodge star The inner orientation of an outer oriented manifold $\mathcal{S}$ changes with a change in ambient orientation (see discussion above). In 
order to find nevertheless integral values over outer oriented manifolds that do not depend on ambient orientation, one defines twisted differential forms $\widetilde{\omega} \in \widetilde{\Omega}^{k}(\mathcal{M})$ by

$$
\widetilde{\omega}:=\{\{\omega, O r\},\{-\omega,-O r\}\},
$$

which provide concrete representations of the twisted forms of Definition 7. Then, the sign and value of the integral $\int_{\mathcal{S}} \widetilde{\omega}$, represented in $O r$ by $\omega$ hence $\int_{\mathcal{S}} \omega$, and in $-O r$ (which leads to opposite inner oriented $\mathcal{S}$ ) by $-\omega$ hence $\int_{-\mathcal{S}}-\omega$, is independent of ambient orientation. In case of maximal degree, namely a twisted $n$-form on a $n$-manifolds, these forms are also called densities (Jänich (2001)).

Besides its metric dependency induced by $\langle\cdot, \cdot\rangle$, the Hodge star operator $\star$ of Eqn. (21) depends on the choice of orientation. This dependency follows because the volume form $\mu$ consists of $n$-basis vectors $d x^{i}, i=1, \ldots, n$, that form a direct frame in $O r$ and a skew frame in $-O r$. Analogously to the definition of twisted forms in Eqn. (78), a formulation independent of the ambient orientation, called twisted Hodge star operator $\widetilde{\star}$, can be found by forming equivalence classes, i.e.

$$
\widetilde{\star} \omega=\{\{\star \omega, O r\},\{-\star \omega,-O r\}\} .
$$

By definition, it follows directly that $\widetilde{\star}$ maps straight $k$-forms to twisted $(n-k)$-forms. On the other hand, if one applies $\widetilde{\star}$ to a twisted $k$-form $\widetilde{\omega}$, one selects for the latter one a representative $\{\omega, O r\}$ and applies to $\omega$ the operator $\star$ that is defined by the selected $O r$. This returns the straight $(n-k)$-form $\widetilde{\star} \widetilde{\omega}$. Analogously to $\star$, the twisted Hodge star fulfills $\widetilde{\star} \widetilde{\omega}=(-1)^{k(n-k)} \omega$ for a $k$-form $\omega$ (Bossavit (1998d)).

Hierarchical structure of spaces and manifolds As summary of Sections 2 and 7.1, we relate the spaces and manifolds and the thereon defined structures and operators. As illustrated in the following commutative diagram, an affine space $A_{n}$ equipped with a metric $\mathbf{g}$ and orientation $O r$ is an oriented Euclidean space $E_{n}$. On the other hand, the (possibly oriented) affine space $A_{n}$ and Euclidean space $E_{n}$ can be seen as local linearizations by the differential $d h_{p}$ of a chart $(U, h)$ of the (possibly oriented) topological manifold $\mathcal{M}$ and Riemannian manifold $(\mathcal{M}, \mathbf{g})$, respectively. That is, the diagram

$$
\begin{aligned}
& \text { topological manifold } \mathcal{M} \stackrel{\mathbf{g}, O r}{\longrightarrow} \text { oriented Riemannian manifold }(\mathcal{M}, \mathbf{g}, \text { Or }) \\
& \qquad d h_{p} \mid \forall p \in U \subset \mathcal{M} \\
& \qquad d h_{p} \downarrow \forall p \in U \subset \mathcal{M}
\end{aligned}
$$

affine space $A_{n} \quad \stackrel{\mathbf{g}, O r}{\longrightarrow} \quad$ oriented Euclidean space $\left(E_{n}, \mathbf{g}\right.$, Or $)$

commutes for spaces endowed with, for instance, vector fields $X \in \mathcal{X}(\mathcal{M})$, straight and twisted differential forms $\omega \in \Omega(\mathcal{M})$ and $\widetilde{\omega} \in \widetilde{\Omega}(\mathcal{M})$, and various operators acting upon those fields. On both $E_{n}$ and $(\mathcal{M}, \mathbf{g})$ exist different equivalent realizations of metric structures. How this latter fact influences the equations of GFD will be subject of Sect. 9.2.

\subsection{Splitting the covariant equations into topological and metric parts}

By reformulating equations (60), we aim to find structured fluid equations that apply only as much mathematical structure as necessary and that better match the geometrical properties of the fluid's quantities of interest.

The basic idea in order to find a structure of the equations that would fulfill this two conditions is to introduce in both momentum and continuity equations auxiliary quantities that do not depend on the metric structure and to take into account that $k$-forms are integrated on 
$k$-manifolds for all $k \leq n$. Then, the evolution of the fluid's momentum along a curve $c \subset \mathcal{M}$ is described by the 1 -form $u \in \Omega^{1}(\mathcal{M})$, by the functions (0-forms) $\rho, p \in \Omega^{0}(\mathcal{M})$ for density and pressure, respectively, by the auxiliary vector field $\vec{V} \in \mathcal{X}(\mathcal{M})$, and by an integration of the momentum equation along $c$. The density evolution in a volume $V \subset \mathcal{M}$ is described by the auxiliary density $n$-form $\widetilde{\rho} \in \Omega^{n}(\mathcal{M})$, by the auxiliary mass-flux $(n-1)$-form $\widetilde{\rho u} \in \Omega^{(n-1)}(\mathcal{M})$, and by the integrations over an $n$ - and $(n-1)$-volume, respectively. As indicated by $\sim$, both auxiliary differential forms used in the continuity equation are twisted ones, as defined in Def. (78).

The resulting equations, in the following called topological equations, only require a topology on $\mathcal{M}$, but no metric. Metric information is contained in additional closure equations, in the following called metric equations. The latter equations apply the metric-dependent operators Riemannian lift $\sharp, b$ of Def. (19) and twisted Hodge star $\widetilde{\star}$ of Def. (79) and they relate the auxiliary quantities with the corresponding original ones, as used in Eqn. (60), in order to close the system of equations.

The suggested reformulation leads to a decoupling between the topological momentum and the topological continuity equation and to an outsourcing of the metric information to additional metric equations, as suggested in the following theorem.

Theorem 3. Let $(\mathcal{M}, \mathbf{g}, O r)$ be a compact, with $\Omega_{\mathrm{rot}}$ uniformly rotating, oriented Riemannian $n$-manifold with smooth boundary $\partial \mathcal{M}$ and let $\Phi_{A^{n}}$ be the corresponding apparent gravity of Theorem 2. Let the fluid's momentum be described by the straight 1-form $u \in \Omega^{1}(\mathcal{M})$, by the straight 0 -forms $\rho, p, \Phi_{A^{n}} \in \Omega^{0}(\mathcal{M})$, and by the vector field $\vec{V} \in \mathcal{X}(\mathcal{M})$, and its density evolution by the twisted $n$-form $\widetilde{\rho} \in \Omega^{n}(\mathcal{M})$ and by the twisted $(n-1)$-form $\widetilde{\rho u} \in \Omega^{(n-1)}(\mathcal{M})$.

Then, the topological momentum and continuity equations in integral form for any static curve $c \subset \mathcal{M}$ or static n-volume $V \subset \mathcal{M}$, given by

$$
\begin{aligned}
& \partial_{t} \int_{c} u+\int_{c} \mathbf{i}_{\vec{V}}\left(\mathbf{d} u+2 \Omega_{\mathrm{rot}}\right)+\int_{\partial c} \frac{1}{2} \mathbf{i}_{\vec{V}} u+\int_{\partial c}\left(w+\frac{p}{\rho}\right)+\int_{\partial c} \Phi_{A^{n}}=0, \\
& \partial_{t} \int_{V} \widetilde{\rho}+\int_{\partial V} \widetilde{\rho u}=0
\end{aligned}
$$

are well-defined on oriented topological manifolds $(\mathcal{M}$, Or) and are independent of the metric $\mathbf{g}$. The metric equations, given by

$$
\widetilde{\star}(\rho u)=\widetilde{\rho u}, \quad \widetilde{\star} \rho=\widetilde{\rho}, \quad u^{\sharp}=\vec{V},
$$

and which uniquely connect the twisted with the straight forms and the velocity 1-form with its proxy, are well-defined on oriented Riemannian manifold $(\mathcal{M}, \mathbf{g}, O r)$ and depend on $\mathbf{g}$.

Both, topological (80) and metric (81) equations are independent of the choice of Or. They provide, in combination with boundary conditions $\mathbf{i}_{u^{\sharp}} \mu$, with initial conditions $u(x, 0)=u_{0}(x)$, and with energy closure for either (i) an incompressible flow (by $\star \mathbf{d} \star u=0)$ or (ii) a barotropic flow (by $p=\rho^{2} \frac{\partial w(\rho)}{\partial \rho}$ with $p=p(\rho)$ and internal energy $w=w(\rho)$ ), a closed set of equations of GFD.

In the following, we refer to this closed set of equations, hierarchically-structured into topological and metric parts, as split Euler's equations of geophysical fluid dynamics.

Remark 18. 1.) The structure of the fluid equations (80) and (81) is similar in form to the covariant Maxwell's equations split into topological and metric parts, as introduced by e.g. Bossavit (1999, 2001).

2.) In contrast to the topological equations (80), the covariant momentum and continuity equations in (60) apply both $\sharp$ and $\star$ and, therefore, depend on $\mathbf{g}$ (see discussion below). 
In case of incompressible and barotropic fluids, one might use in the momentum equations (45), (60), and (80) the pressure term $\mathbf{d} h$, with enthalpy $h=w+p / \rho$, instead of $(1 / \rho) \mathbf{d} p$, as shown in the following lemma (cf. also Abraham et al. (1983)).

Lemma 2. Let the pressure $p=p(\rho)$ and the internal energy $w=w(\rho)$ be functions of $\rho$. Then, in case of incompressible or barotropic fluids, there exists the following identity for the pressure term:

$$
\mathbf{d} h=\frac{1}{\rho} \mathbf{d} p \quad \text { with enthalpy } \quad h:=\left(w+\frac{p}{\rho}\right),
$$

where the internal energy is given by $w=-\int p \mathbf{d}\left(\frac{1}{\rho}\right)$ (with $w=0$ in case of incompressible flow).

Proof. Under the conditions that both $p=p(\rho)$ and $w=w(\rho)$ are functions of $\rho$ alone, the internal energy $w$ is determined by the equation of state $\frac{1}{\rho^{2}} p(\rho)=\frac{\partial w(\rho)}{\partial \rho}$. This follows from integrating the latter equation on both sides over $\rho$, and substituting $\frac{1}{\rho}:=\rho \Rightarrow \frac{\mathbf{d}(1 / \rho)}{\mathbf{d} \rho}=-\frac{1}{\rho^{2}}$ on the left-hand side by using the separation of variables, i.e.

$$
\int \frac{p(\rho)}{\rho^{2}} \mathbf{d} \rho=\int \frac{\partial \omega(\rho)}{\partial \rho} \mathbf{d} \rho \Rightarrow-\int p \mathbf{d}(1 / \rho)=\int \frac{\partial \omega(\rho)}{\partial \rho} \mathbf{d} \rho=w
$$

Setting for the integrand $\mathbf{d} \omega:=-p \mathbf{d}(1 / \rho)$, one finds the identity for the pressure term by

$$
\mathbf{d}(p / \rho)=1 / \rho \mathbf{d} p+p \mathbf{d}(1 / \rho)=1 / \rho \mathbf{d} p-\mathbf{d} \omega \quad \Rightarrow \quad \frac{1}{\rho} \mathbf{d} p=\mathbf{d}(\omega+p / \rho)=\mathbf{d} h .
$$

In case of incompressible flows, this identity follows directly by the continuity equation, i.e. by $\operatorname{div}(\vec{v})=0 \Rightarrow \mathbf{d} \rho=0 \Rightarrow w=0$, and thus $\mathbf{d}(p / \rho)=\frac{1}{\rho} \mathbf{d} p=\mathbf{d} h$.

Proof of Theorem 3. To show that the split Euler's equations (80) and (81) provide a closed set of equations of GFD, we reformulate them and compare them with those given in Theorem 2 . Besides the following proof, we provide in Sect. 7.3 a detailed geometrical interpretation of these equations.

As seen in Sect. 2.1.3, integration over forms is well-defined on oriented topological manifolds. The integrals of both momentum and continuity equations are calculated over static curves or static $n$-volumes, respectively, because the dynamical aspect of the moving fluid has already been taken into account. The latter fact follows directly as we use the Eulerian form of the fluid equations (cf. Eqn. (39)). This allows us to integrate also over the tendencies $\int_{c} \partial_{t} u$ and $\int_{V} \partial_{t} \widetilde{\rho}$. Using Stokes' theorem (16), we replace in the momentum equation the integrals of function $f$ over $\partial c$ by integrals of $\mathbf{d} f$ over $c$, i.e. $\int_{c} \mathbf{d} f=\int_{\partial c} f$. Analogously, we proceed for the mass-flux, i.e. $\int_{V} \mathbf{d} \widetilde{\rho u}=\int_{\partial V} \widetilde{\rho u}$. Substituting the metric equations for a given orientation $O r$ into the topological equations, we find the local form of the fluid equations by

$$
\partial_{t} u+\mathbf{i}_{u^{\sharp}}\left(\mathbf{d} u+2 \Omega_{\mathrm{rot}}\right)+\frac{1}{2} \mathbf{d i}_{u^{\sharp}} u+\mathbf{d}\left(w+\frac{p}{\rho}\right)+\mathbf{d} \Phi_{A^{n}}=0, \quad \partial_{t} \star \rho+\mathbf{d} \star \rho u=0,
$$

as, according to Definition 7, the limits $c \rightarrow 0$ for the momentum and $V \rightarrow 0$ for the continuity equation exist. This set of local fluid equations also follows in case of $-O r$, because then both terms of the continuity equation carry a minus sign (see discussion in Sect. 7.3). Because of Lemma 2, one sees immediately that this local form agrees with the $n$-dimensional fluid equations (60), which proves that equations (80) and (81) provide, with the corresponding closure equations, a closed set of equations of GFD. 
In the momentum equation only straight forms and in the continuity equation only twisted forms are used. The metric equations (81) connect straight and twisted forms via the twisted Hodge star operator $\widetilde{\star}$ defined in $(79)$. By its definition, $\widetilde{\star}$ connects uniquely straight $k$-forms with twisted $(n-k)$-forms, i.e. $\widetilde{\star}$ uniquely maps $\rho$ to $\widetilde{\rho}$ and $(\rho u)$ to $\widetilde{\rho u}$ (cf. Sect. 7.3). The fact that $\widetilde{\star}$ is a unique map can be seen as follows. Assume that $\widetilde{\star}$ maps the $k$-form $\omega$ to the $(n-k)$-form $\widetilde{\star} \omega$. Assume further that $\widetilde{\star} \bar{\omega}$ is another $(n-k)$-form with $\widetilde{\star} \bar{\omega} \neq \widetilde{\star} \omega$ resulting from this mapping. As by definition, $\widetilde{\star} \bar{\omega}=\widetilde{\star}\left(\omega_{1, \ldots, k} d x^{1} \wedge \ldots \wedge d x^{k}\right)=\omega_{1, \ldots, k} \widetilde{\star} d x^{1} \wedge \ldots \wedge d x^{k}=( \pm) \omega_{1, \ldots, k} d x^{k+1} \wedge \ldots \wedge d x^{n}=\widetilde{\star} \omega$ (both $\widetilde{\star} \omega$ and $\widetilde{\star} \bar{\omega}$ share the same sign following from the choice of orientation), the equality of $\widetilde{\star} \omega$ and $\widetilde{\star} \bar{\omega}$ leads to a contradiction, which proves the uniqueness of $\widetilde{\star}$ (cf. Sect. 7.3.2). Hence, the split Euler's equations are well-defined on oriented Riemannian manifolds.

By introducing metric-independent auxiliary quantities, the metric-dependent operators $\star, \sharp$, as used in Eqn. (60), are transferred from the topological equations (80) to the metric equations (81). Then, in the topological equations only topological operators, such as the exterior derivative $\mathbf{d}$ and the interior product $\mathbf{i}$, act on affine vector fields and on differential forms, which are defined in purely topological manner (cf. Sect. 2.1.2). Therefore, the topological equations (80) and the quantities of interest such as velocity $u$, vorticity $\mathbf{d} u$, and density $\widetilde{\rho}$ described by differential forms are well-defined on the topological manifold $\mathcal{M}$. The integration of these equations, though, requires that the manifold $\mathcal{M}$ is oriented. By using the suggested combination of straight and twisted differential forms, both equations in (80) are nevertheless independent of the choice of orientation (cf. Sect. 7.3.1).

The metric dependency of the metric equations follows directly by the definitions of the Riemannian lift $b, \sharp$ in (19) and of the twisted Hodge star $\widetilde{\text { in }}(79)$. In both cases, an inner product $\langle\cdot, \cdot\rangle$ that depends on the chosen metric structure $\mathbf{g}$ is required. For a given inner product, one finds a unique isomorphism $b, \sharp$ that connects 1 -forms with their vector proxies, i.e. $b: V \rightarrow V^{*}, \vec{v} \mapsto\langle\vec{v}, \cdot\rangle$ for a vector space $V$, and a unique isomorphism $\star, \widetilde{\star}$ between $k$-forms and $(n-k)$-forms, i.e. $\star: \Omega^{k}(\mathcal{M}) \rightarrow \Omega^{(n-k)}(\mathcal{M})$ with $\alpha \wedge \star \beta=\langle\alpha, \beta\rangle \mu$ for $\alpha, \beta \in \Omega^{k}(\mathcal{M})$. Choosing a different metric structure $\mathbf{g}$ on $\mathcal{M}$, other, but also unique, isomorphisms $b, \sharp, \star$, and $\widetilde{\star}$ exist; they differ from each other with respect to $\mathbf{g}$. Therefore, the metric equations are well-defined on $(\mathcal{M}, \mathbf{g})$ and depend on the chosen metric $\mathbf{g}$.

Corollary 5. Given the assumptions of Theorem 3 except that an orientation of $\mathcal{M}$ is not needed, the topological momentum and continuity equations in local form are given by

$$
\partial_{t} u+\mathbf{i}_{\vec{V}}\left(\mathbf{d} u+2 \Omega_{\text {rot }}\right)+\frac{1}{2} \mathbf{d i}_{\vec{V}} u+\frac{1}{\rho} \mathbf{d} p+\mathbf{d} \Phi_{A^{n}}=0, \quad \partial_{t} \widetilde{\rho}+\mathbf{d} \widetilde{\rho u}=0 .
$$

They require no metric and no orientation on topological manifolds $\mathcal{M}$ and provide, with the metric equations (81) and the corresponding closure conditions, a closed set of local split Euler's equations of GFD.

Proof. The Corollary follows from Theorem 3 and from the fact that straight and twisted $k$ forms, to be well-defined, do not require orientation on a topological manifold (cf. Sect. 2.1.3).

\subsection{Geometrical interpretation of the split Euler's equations of GFD}

In this section, we present a geometrical interpretation of the structure provided by the split Euler's equations of GFD and we show how the straight and twisted $k$-forms used in Eqns. (80) match in dimension and orientation the corresponding physical quantities of the fluid, such as velocity, density, and mass-flux. In addition, we illustrate how the Hodge star operator connects straight and twisted forms, how the Riemannian lift $b, \sharp$ connects the velocity 1 -form with its 
vector proxy, and how this relates to the split equations. To discuss physically realistic fluid flows, we restrict our discussion to the split Euler's equations of GFD in $\mathbb{R}^{3}$, and we provide SI-units for the fluid's quantities of interest.

\subsubsection{The metric-free topological momentum and continuity equations}

The use of differential forms in the split Eulers' equations and their separation into topological and metric parts, as given in Theorem 3, allow us to geometrically interpret the momentum and continuity equations (80) separately, as indicated in Fig. 3.

The topological momentum equation In the momentum equation, the fluid's velocity is described by the straight 1-form $u: c \rightarrow \int_{c} u$, which measures the alignment of the velocity field $u$ along an inner oriented curve $c \in \mathcal{M}$. Its time derivative $\partial_{t} u$ describes the work performed by $u$ along curve $c$ in $\left[\mathrm{m}^{2} / \mathrm{s}^{2}\right]=[\mathrm{J} / \mathrm{kg}]$ and thus corresponds to the definition of a specific energy density, analogously to the internal energy $w$ in Eqn. (33). This agrees with the interpretation of forces as 1-forms that assign to the displacement of a particle an amount of work performed on this particle (cf. Sect. 2.2.2).

The fluid's rate of rotation within an area $A$, given in $\left[\mathrm{m}^{2} / \mathrm{s}\right]$, is represented by the straight 2-form $\mathbf{d} u: A \rightarrow \int_{A} \mathbf{d} u$ which corresponds, via Stokes' theorem $\int_{A} \mathbf{d} u=\int_{\partial A} u$, to the fluid's circulation along this area's boundary $\partial A$. The sign of $\int_{A} \mathbf{d} u$ describes the sense of rotation and depends only on the inner orientation of $A$. The corresponding point value of $\mathbf{d} u$ is called vorticity and is given in the metric-free units $[1 / s]$. The advection of the vorticity $\mathbf{d} u$ in the direction of $\vec{V}$ leads to a work performed along $c$. This flow feature can be adequately represented by the straight 1-form $\mathbf{i}_{\vec{V}} \mathbf{d} u: c \rightarrow \int_{c} \mathbf{i}_{\vec{V}} \mathbf{d} u$ in units of $\left[\mathrm{m}^{2} / \mathrm{s}^{2}\right]$. The same argumentation holds for $\mathbf{i}_{\vec{V}} 2 \Omega_{\text {rot }}$.

The straight 0 -forms $h, \kappa$, and $\Phi_{A^{n}}$, using the definitions of enthalpy $h=w+p / \rho$ in Eqn. (82) and of kinetic energy $\kappa=: \frac{1}{2} \mathbf{i}_{\vec{V}} u$ for $\vec{V}=u^{\sharp}$, describe potentials in units of $\left[\mathrm{m}^{2} / \mathrm{s}^{2}\right]$. The potential difference between the boundaries $\partial c$ leads to work performed along curve $c$ (cf. Fig. 3 (left)). In case of a closed loop $c=\partial A$, the potential difference is zero, leading to the conservation of absolute circulation $(d / d t) \int_{A} \omega_{a}=0$ with absolute vorticity $\omega_{a}:=\left(\mathbf{d} u+2 \Omega_{\text {rot }}\right)$ (cf. Sect. 8 for more details).

The inner orientation of $c$ assigns a direction to the fluid flow and determines the sign of the integral $\int_{c} u$. The signs of the potentials at the boundaries $\partial c$ are determined by the inner orientations of the boundary points. The latter, in turn, can be consistently derived by the inner orientation of the curve $c$ (cf. Sect 2.1.3). That is, we assume $c$ to be its own ambient space. Then, the crossing direction from inside $c$ to the outside outer orients its boundary points. This enables to assign to these points inner orientations. The boundary point where the inner orientation of $c$ and the outer orientation of $\partial c$ point in the same direction is inner oriented with $(+)$ and denoted by $\partial c_{+}$. The other boundary point with opposite direction to $c$ is inner oriented with $(-)$ and denoted by $\partial c_{-}$(see Fig. 3 (left)). In case of opposite inner orientation of $c$, both boundary points change sign, leaving the topological momentum equation invariant under changing inner orientation. The inner orientation of $c$ is here independent of the orientation $\mathrm{Or}$ of a surrounding ambient space.

In agreement with the proof to Theorem 3, this discussion illustrates that the topological momentum equation is independent of the choice of orientation $O r$, as all differential forms used are straight and thus act, according to Definition 7, on inner oriented manifolds. Moreover, as indicated by the units $[\mathrm{J} / \mathrm{kg}]$ of a specific energy density, the topological momentum equation is metric independent. 


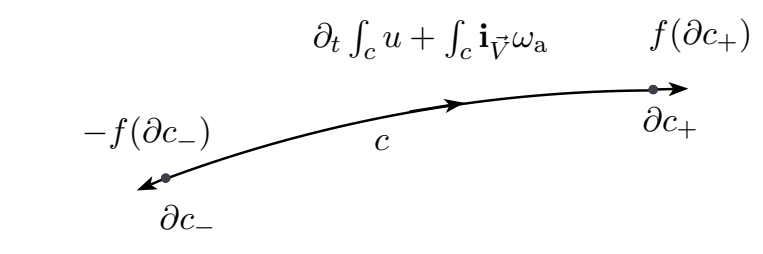

$\partial_{t} \int_{c} u+\int_{c} \mathbf{i}_{\vec{V}}(\underbrace{\mathbf{d} u+2 \Omega_{\mathrm{rot}}}_{=: \omega_{\mathrm{a}}})+\int_{\partial c}(\underbrace{\frac{p}{\rho}+w+\kappa+\Phi_{A^{n}}}_{=: f})=0$

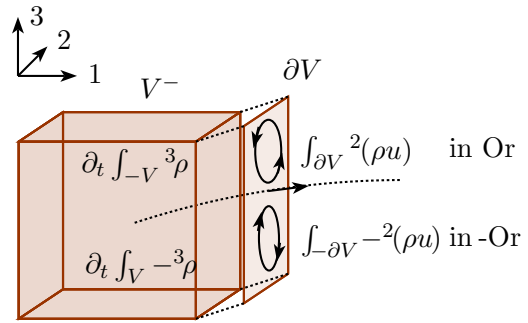

$\partial_{t} \int_{V} \widetilde{\rho}+\int_{\partial V} \widetilde{\rho u}=0$

Figure 3: Geometrical interpretation of the topological equations. Left: the work $\partial_{t} \int_{c} u$ in $[J / k g]$ performed along $c$ is caused by the potential differences $f\left(\partial c_{+}\right)-f\left(\partial c_{-}\right)$and by the advected absolute vorticity $\int_{c} \mathbf{i}_{\vec{V}} \omega_{\mathrm{a}}$. Right: the mass change $\partial_{t} \int_{V} \widetilde{\rho}$ in $[\mathrm{kg} / \mathrm{s}]$ arises from the mass-flux $\int_{\partial V} \widetilde{\rho u}$ over the boundary $\partial V$ of volume $V^{-}$.

The topological continuity equation In the continuity equation, the mass of a portion of fluid with volume $V$ is described by the twisted 3-form $\widetilde{\rho}: V \rightarrow \int_{V} \widetilde{\rho}$ which assigns to $V$ its mass in units of $[\mathrm{kg}]$. Its time derivative $\partial_{t} \widetilde{\rho}$ describes, in $[\mathrm{kg} / \mathrm{s}]$, the change in mass within $V$. This change is caused by the mass-flux, i.e. by the amount of mass that traverses per unit of time the boundary $\partial V$ of $V$. This mass-flux, given in units of $[\mathrm{kg} / \mathrm{s}]$, is described by the twisted 2-form $\widetilde{\rho u}: \partial V \rightarrow \int_{\partial V} \widetilde{\rho u}$ (cf. Fig. 3 (right)). As indicated by these units, the topological continuity equation is independent of any metric structure (in agreement with Theorem 3).

The notion of "flux through the surface $\partial V$ " outer orients this surface by a crossing direction. In order to meet the sign convention in equations (80), this mass-flux must be directed from a region that is outer oriented with $(-)$ to a region outer oriented with $(+)$. We denote the region outer oriented with $(-)$ by $V^{-}$and with $(+)$by $V^{+}$(cf. Fig. 4). The crossing direction of $\partial V$ points from $V^{-}$toward $V^{+}$, which corresponds in Fig. 3 (right) to a mass-flux from inside $V^{-}$to the outside. Depending on the ambient orientation, an outer orientation determines a region's inner orientation. The outer orientation $(-)$ of $V^{-}$, for instance, induces a negative inner orientation in $O r$ giving a negative integral $-\int_{V^{-}}{ }^{3} \rho$ for the positive representative ${ }^{3} \rho$. We use the upper index to indicate the differential form's dimension. Using twisted forms, the same integral follows in $-O r$ as the inner orientation of $V$ changes sign, which is compensated by the negative representative $-{ }^{3} \rho$.

The crossing direction induces an inner orientation of $\partial V$, which depends on the ambient orientation, too. In $O r$, this inner orientation is positive giving a positive mass-flux $\int_{\partial V}{ }^{2}(\rho u)$ from inside $V^{-}$to the outside for the positive representative ${ }^{2}(\rho u)$. In case of $-O r$ and of fixed crossing direction, the inner orientation of $\partial V$ changes sign (cf. Fig. 3 (right)). Here as well, this change in the sign of the integral is compensated by the negative representative $-{ }^{2}(\rho u)$. This illustrates the orientation independent definition of the topological continuity equation (80).

Remark 19. The integral form of the split Euler's equations of GFD (80) requires consistent orientations of the inner oriented curve $c$, of the outer oriented volume $V$, and of their boundaries. In this paragraph, we have introduced such consistently oriented manifolds that match the sign convention of Eqns. (80). Because the local split Euler's equations of GFD (86) are not integrated over manifolds, they do not require the structure of orientation on $\mathcal{M}$ and are therefore, in this sense, more general. 


\subsubsection{The metric-dependent closure equations}

As indicated by their name, the metric-dependent closure equations (81) aim (i) to equip the split Euler's equations of GFD with information about metric and (ii) to close the set of equations.

The metric information follows by using the twisted Hodge star operator $\widetilde{\star}$ of Eqn. (79) and the Riemannian lift $\sharp, b$ of Eqn. (19) as both operators rely on the inner product that depends on the metric structure g. However, both are independent of the choice of ambient orientation. More concretely, the Hodge star $\star: \omega \wedge \star \omega=\langle\omega, \star \omega\rangle \mu$, mapping a $k$-form $\omega$ to a $(n-k)$-form $\star \omega$, applies the inner product and depends thus on the metric. As $\star$ applies, in addition, the volume form $\mu$ which consists of an $n$-basis that belongs to one of the two orientation classes, $\star$ depends on the orientation too. To avoid such dependency, we use the twisted Hodge star $\widetilde{\star}$ of Def. (79) which is independent of orientation (see discussion below). The Riemannian lift $\sharp: \omega=\langle\vec{w}, \cdot\rangle \rightarrow \vec{w}$ maps a metric-independent 1-form $\omega$ to its vector proxy field $\vec{w}$ that depends on the chosen metric structure, although vector fields are, in principle, affine object (cf. Sect. 2.1.1). \# maps straight or twisted 1-forms to straight or twisted vector proxies, respectively, independently of ambient orientation.

These operators are used to close the set of topological fluid equations (80). The metric equations using the twisted Hodge star connect the initially independent topological momentum with the continuity equation, as shown in Fig. 4. These equations have to be fulfilled in order to obtain a well-defined set of fluid equations. The metric equation that applies the Riemannian lift, i.e. $u^{\sharp}=\vec{V}$, is confined to the topological momentum equation. In case this closure condition is fulfilled in all terms, there follow the full nonlinear Euler's equations of GFD. However, an approximation of the latter condition, particularly when applied on the advection term to obtain a set of linearized fluid equations, is possible. In the following, we discuss these different cases in more detail.

The metric equations applying the twisted Hodge star To obtain a well-defined, closed set of fluid equations, the initially independent topological momentum and continuity equations (cf. Fig. 3) have to be connected with each other uniquely and in a consistent way, as illustrated in Fig. 4, by those metric equations in (81) that apply the twisted Hodge star operator $\widetilde{\star}$.

For the general $n$-dimensional split Euler's equations of GFD, given in (80) or (86), the twisted Hodge star $\widetilde{\star}$ provides, according to Def. (79) and Theorem 3, a unique mapping between the density function (0-form) $\rho$ and the twisted $n$-form $\widetilde{\rho}$ by $\widetilde{\star}: \rho \mapsto \widetilde{\rho}:=\left\{\left\{{ }^{n} \rho, O r\right\},\left\{-{ }^{n} \rho,-O r\right\}\right\}$. In other words, for a given density $\rho$, there exist two straight $n$-forms, namely ${ }^{n} \rho$ for $O r$ and $-{ }^{n} \rho$ for $-O r$, whereas there is only one twisted $n$-form $\widetilde{\rho}$. Analogously, $\widetilde{\star}$ provides a unique mapping between the straight 1 -form $\rho u$ and the twisted $(n-1)$-form $\widetilde{\rho u}$ by $\widetilde{\star}:(\rho u) \mapsto \widetilde{\rho u}:=$ $\left\{\left\{{ }^{(n-1)}(\rho u), O r\right\},\left\{-^{(n-1)}(\rho u),-O r\right\}\right\}$. In the following, we illustrate on a concrete example in $\mathbb{R}^{3}$ the uniqueness and consistency of $\widetilde{\star}$.

As shown in Fig. 4, the twisted Hodge star $\widetilde{\star}$ maps the straight density function $\rho$ in units of $\left[\mathrm{kg} / \mathrm{m}^{3}\right]$ to the twisted 3 -form $\widetilde{\rho}$ in units of $[\mathrm{kg}]$. Analogously, the straight 1-form $(\rho u)$ in units of $[\mathrm{kg} /(\mathrm{m} \cdot \mathrm{s})]$ is mapped to the twisted 2 -form $\widetilde{\star}(\rho u)$ in units of $[\mathrm{kg} / \mathrm{s}]$. In both cases, $\widetilde{\star}$ maps from metric-dependent to metric-independent forms by amending the former with the required metric information. This shows that the twisted Hodge star $\widetilde{\star}$ consistently connects the metricfree momentum equation, given in units of $[\mathrm{J} / \mathrm{s}]$, with the metric-free continuity equation, given in units of $[\mathrm{kg} / \mathrm{s}]$.

In addition, the twisted Hodge star $\widetilde{\star}$ takes care of a consistent and from the ambient orientation independent connection between the inner oriented momentum and the outer oriented continuity equation, i.e. the inner orientations of $c$ and $\partial c$ consistently outer orient $V$ and $\partial V$, respectively (cf. Fig. 4). To see this, recall that the inner orientation of $c$ induces canonical 

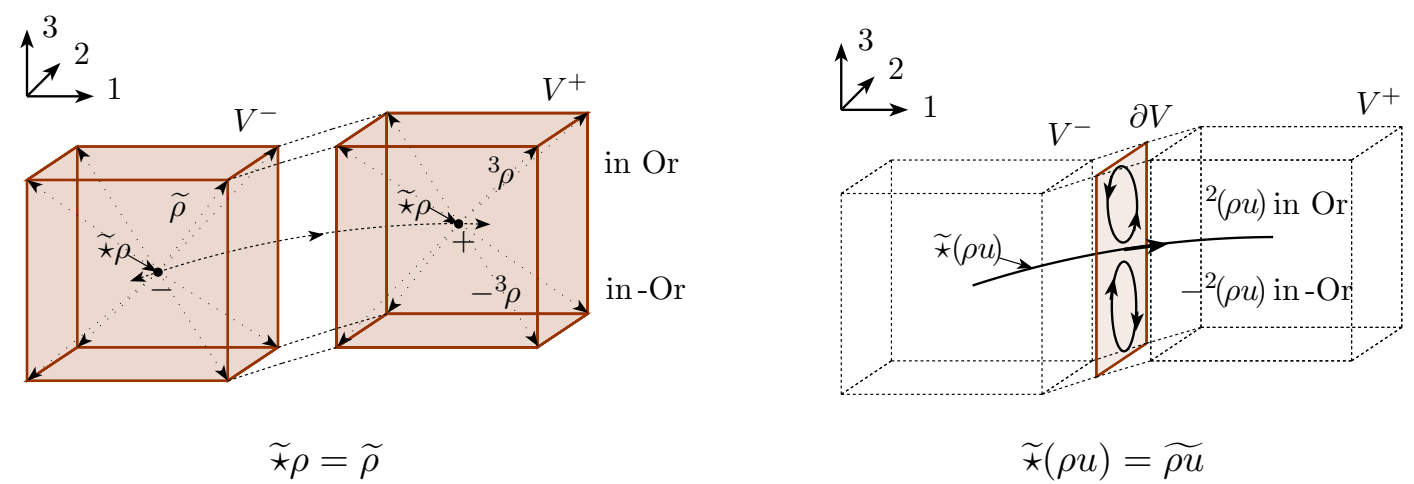

Figure 4: Geometrical interpretation of the metric equations. Left: $\widetilde{\star}$ maps the 0 -form $\rho$ in $\left[\mathrm{kg} / \mathrm{m}^{3}\right]$ to the two representative 3 -forms $\left\{{ }^{3} \rho, O r\right\}$ and $\left\{-{ }^{3} \rho,-O r\right\}$ in $[\mathrm{kg}]$. Right: $\widetilde{\star}$ maps the 1 -form $(\rho u)$ in $[\mathrm{kg} /(\mathrm{m} \cdot \mathrm{s})]$ to the two representative 2 -forms $\left\{{ }^{2}(\rho u), O r\right\}$ and $\left\{-{ }^{2}(\rho u),-O r\right\}$ in $[\mathrm{kg} / \mathrm{s}]$.

inner orientations of its boundary points, i.e. (+) for $\partial c_{+}$and $(-)$for $\partial c_{-}$(cf. Fig. 3 (left)). These orientations are independent of ambient orientation (cf. Sect. 7.3.1). Then, the inner orientation of each boundary point orients its surrounding volume by the same sign, i.e. the volume around $\partial c_{-}$is outer oriented with $(-)$(denoted with $V^{-}$according to Sect. 7.3.1) and the volume around $\partial c_{+}$with $(+)$(i.e. $V^{+}$). The resulting crossing direction of $\partial V$, pointing from $V^{-}$toward $V^{+}$in Fig. 4, coincides with the inner orientation of the curve $c$ (see Fig. 3) and is independent of the ambient orientation.

As further discussed in Sect. 7.3.1, the integral of the topological momentum equation over the inner oriented curve $c$ and over its boundary points is independent of ambient orientation. As just argued, the outer orientations of the volumes $V^{-}$and $V^{+}$and the crossing direction, both induced by $c$, remain unchanged too with changing orientation. The inner orientations of these volumes (cf. Fig. 4 (left)) and of the boundary $\partial V$ between these volumes (cf. Fig. 4 (right)), on the other hand, change. These changes in inner orientations are however compensated by the twisted forms (cf. again Sect. 7.3.1) which make the integral of the continuity equations over the outer oriented volume $V$ with its outer oriented boundary $\partial V$ independent of orientation too. Hence, besides providing the required metric information, the twisted Hodge star $\widetilde{\star}$ connects the topological equations uniquely and independently of ambient orientation.

The metric equation applying the Riemannian lift In the metric equation (81) (right), the Riemannian lift $\sharp$ maps the 1 -form $u$ describing the fluid's velocity to its vector proxy $\vec{u}$ by $\sharp: u=\langle\vec{u}, \cdot\rangle \mapsto \vec{u}$. Here, we distinguish between the notation $\vec{u}$, in case the latter closure condition is fulfilled, and the general (auxiliary) vector field $\vec{V}$, used in the topological momentum equation (80). The mapping $\sharp$ may serve, in a diagnostic step, to regain from $u$ the conventional descriptor for the fluid's velocity by the vector field $\vec{u}$. However, this vector field depends on the metric, since $\sharp$ does.

The metric dependency of this mapping can be illustrated on the topological momentum equation. That is, if we map by $\sharp$ the terms (e.g. $\partial_{t} u$ ) of the covariant momentum equation in units of specific energy density $[\mathrm{J} / \mathrm{kg}]$ to the corresponding vector proxies, the resulting terms (e.g. $\left.\partial_{t} \vec{u}\right)$ of the corresponding vector-valued equation carry the units of a momentum $[\mathrm{J} / \mathrm{kg} / \mathrm{m}]=\left[\mathrm{m} / \mathrm{s}^{2}\right]$ and depend obviously on the metric. This latter dependency is shared by the vector proxy $\vec{u}$ describing the fluid's velocity in units of $[\mathrm{m} / \mathrm{s}]$ (cf. Fig. 5 (left)). 

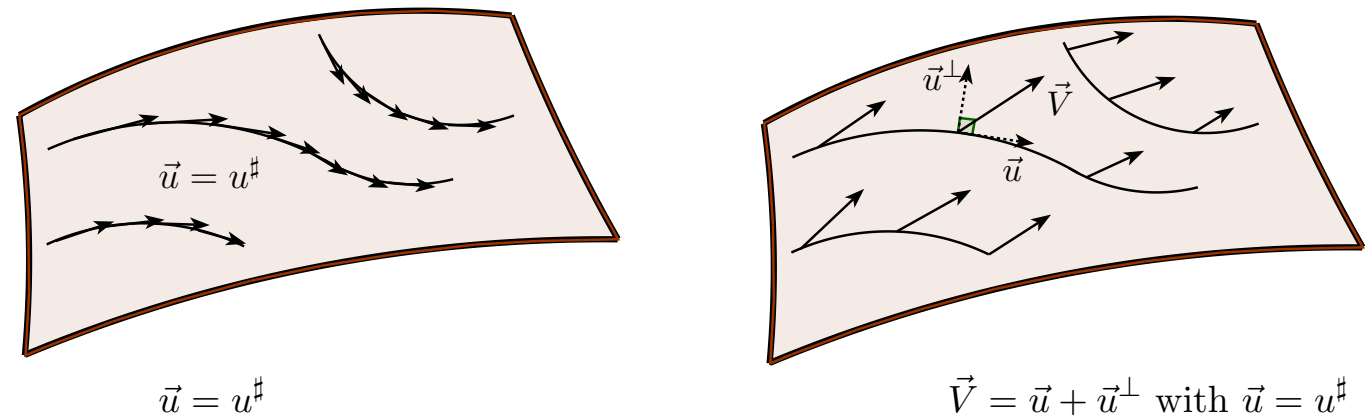

Figure 5: Geometrical interpretation of the metric equations. Left: $\sharp$ maps from the metric-free velocity 1 -form $u$ in $[\mathrm{J} \cdot \mathrm{s} / \mathrm{kg}]$ to metric-dependent velocity proxy field $\vec{u}$ in $[\mathrm{m} / \mathrm{s}]$. Right: General vector field $\vec{V}$ consists of the vector proxy $\vec{u}=u^{\sharp}$ and of a to $\vec{u}$ perpendicular part $\vec{u}^{\perp}$.

Furthermore, we discuss those terms of the momentum equation (80) applying the interior product $\mathbf{i}$ because they depend on the Riemannian lift $\sharp$. According to Sect. 2.2.2, the 1-form $u$ can be interpreted as an operator that assigns to a (virtual) displacement $\delta \vec{v}$ an amount of (virtual) work $u(\delta \vec{v}) \in \mathbb{R}$. Analogously, we can interpret the 2 -form $\mathbf{d} u$ as an operator that assigns to the advection of the vortex $\mathbf{d} u$ along $\delta \vec{v}$ an amount of (virtual) work $\mathbf{i}_{\delta \vec{v}} \mathbf{d} u \in \mathbb{R}$.

In case of $\delta \vec{v}=\vec{u}=u^{\sharp}$, the fluid's velocity $\vec{u}$ itself advects the vortex $\mathbf{d} u$, leading to the nonlinear term $\mathbf{i}_{\vec{u}} \mathbf{d} u$. For the kinetic energy, there follows the straight 0 -form $\kappa=\frac{1}{2} \mathbf{i}_{\vec{u}} u=\sum_{i} \frac{1}{2} u_{i}^{2}$ of Eqn. (73). This recovers the full nonlinear Euler's equations. In case of a general vector field $\delta \vec{v}=\vec{V} \neq \vec{u}$ that is independent of $\vec{u}$, the linear term $\mathbf{i}_{\vec{V}} \mathbf{d} u$ describes the linear advection of the vortex $\mathbf{d} u$ by some background vector field $\vec{V}$, which could be, for instance, some external vector field or some average of the vector proxy field. To our knowledge, there exists no similar interpretation for the term $\mathbf{i}_{\vec{V}} u$.

For a more detailed discussion about the general vector field $\vec{V}$, we represent the latter in terms of the fluid's velocity field $\vec{u}$, i.e. $\vec{V}=\vec{u}+\vec{u}^{\perp}$ with $u^{\sharp}=\vec{u}$ and $\left\langle\vec{u}, \vec{u}^{\perp}\right\rangle=0$ (cf. Fig. 5 (right)). By linearity of the interior product, the advection term can be separated into $\mathbf{i}_{\vec{V}} \mathbf{d} u=\mathbf{i}_{\vec{u}} \mathbf{d} u+\mathbf{i}_{\vec{u} \perp} \mathbf{d} u$. Here, only the part parallel to $\vec{u}$ contributes to the energy budget of the fluid, whereas the perpendicular part $\mathbf{i}_{\vec{u}^{\perp}} \mathbf{d} u$ only describes virtual work. Analogously, there is $\mathbf{i}_{\vec{V}} u=\mathbf{i}_{\vec{u}} u+\mathbf{i}_{\vec{u}} \perp u$. In this case, only the part parallel to the velocity $\vec{u}$ contributes to the fluid's kinetic energy that is given by $\kappa=\frac{1}{2} \mathbf{i}_{\vec{u}} u$. The perpendicular part describes a virtual work performed by a force, for instance the Coriolis force, that acts perpendicularly to a particle's flow direction and thus changes its direction but does not contribute to the kinetic energy, i.e. $\mathbf{i}_{\vec{u}^{\perp}} u=0$.

\subsubsection{Differential forms mimic measurement devices}

Recall that differential forms are linear operators that assign real numbers to manifolds with finite length, area, or volume. Analogously, measurement devices assign measured values of the fluid's quantities of interest such as velocity, vorticity, or mass to finite curves, areas, or volumes.

For instance, a device that measures the fluid's mass, assigns to a portion of fluid contained in a volume its mass in $[\mathrm{kg}]$, similarly to $\widetilde{\rho}$. Another device measures the mass-flux through the boundary of this volume in $[\mathrm{kg} / \mathrm{s}]$, similarly to $\widetilde{\rho u}$. Different approaches that physically measure these quantities (see e.g. Baker (2005)) share the fact that they measure over finite areas and volumes, but not point-wise values, as these latter cannot be measured directly (Bossavit (2005)). 
The velocity of a real fluid is often measured by using the so-called velocimetry ${ }^{6}$, in which the fluid is seeded with tracer particles that follow the fluid flow. The comparison of images of these particles for different times, with possibly short (but nevertheless finite) time intervals, enables to determine the fluid's velocity field. Also the vorticity of the fluid can be measured in such a way (Wallace (1986)). In case of measurements of the potentials, only their (finite) difference between two distinct points can be measured by a measurement device. Analogously in the integral equation (80), $u$ assigns to a given oriented curve the fluid's flow speed and its direction, $\mathbf{d} u$ assigns to an area its circulation and vorticity, and the potential difference is determined between the boundaries of the curve (cf. Sect. 7.3.1 for the corresponding SI-units).

It seems thus justified to interpret the differential forms in the integral form of the split Euler's equations (80) as measurement devices. In contrast, as point-wise values cannot be measured directly, there is no similar geometrical interpretation for the local form of these equations as given in (86).

\section{Conservation properties of the (split) covariant equations of GFD}

In this section, we investigate the $n$-dimensional covariant equations of GFD with respect to their conservation of circulation, vorticity, and potential vorticity. The results are general to the extent that they include all covariant equations introduced in this manuscript. The conservation of these quantities is of outstanding importance in geophysical fluid dynamics. For instance, potential vorticity conservation is the main reason for a splitting between balanced motion and waves. Besides offering such useful insight in the properties of the fluid flow, the conservation of these quantities is also of practical importance, for instance when constructing structure-preserving discretizations of these equations.

\subsection{The $n$-dimensional covariant vorticity equation}

The relative vorticity 2 -form $\omega_{r} \in \Omega^{2}(\mathcal{M})$ results from an exterior derivation of the velocity 1-form $u$, i.e. $\omega_{r}=\mathbf{d} u$ (cf. Sect. 5). Its time evolution is described by the covariant vorticity equation which results from applying $\mathbf{d}$ on the local covariant momentum equation (86), i.e.

$$
\partial_{t} \mathbf{d} u+\mathbf{d i}_{\vec{V}}\left(\mathbf{d} u+2 \Omega_{\mathrm{rot}}\right)=-\mathbf{d d}\left[\frac{1}{2} \mathbf{i}_{\vec{V}} u\right]-\mathbf{d}\left[\frac{1}{\rho} \mathbf{d} p\right]-\mathbf{d} \mathbf{d} \Phi_{A^{n}}=\frac{\mathbf{d} \rho \wedge \mathbf{d} p}{\rho^{2}}
$$

where the rightmost equality follows from the fact that $\mathbf{d} \mathbf{d}=0$. In case of baroclinic fluids, the right-hand side of (87) is nonzero (cf. Remark 20). In case of barotropic and incompressible fluids, the right-hand side is zero, as, according to Lemma (2), there follows $\mathbf{d}[(1 / \rho) \mathbf{d} p]=$ $\mathbf{d d}(w+p / \rho)=0$.

To modify the second term on the left-hand side of (87), we consider for the absolute vorticity 2 -form, defined as $\omega_{a}:=\omega_{r}+\omega_{p}$ with planetary vorticity 2 -form $\omega_{p}:=2 \Omega_{\mathrm{rot}}$, a representation in a local coordinate chart, i.e. $\omega_{a}=\sum_{i<j} \omega_{a \mid i j} d x^{i} \wedge d x^{j}$. Then, with $\vec{V}=\sum_{i} V^{i} \partial_{i}$ and $d x^{i}\left(\partial_{k}\right)=\delta_{i k}$,

\footnotetext{
${ }^{6}$ One distinguish between Particle Image Velocimetry (PIV) and Particle Tracking Velocimetry (PTV), see, e.g., Holenberg et al. (2012). In PIV, different images of the fluid seeded with particles are compared to different times to determine the fluid's velocity. In PTV, the velocity follows from the recorded tracks of the fluid particles. In both cases, measurements over too short time intervals are not possible, because in this case the differences in the images would not be visible.
} 
there follows for $1 \leq i<j \leq n ; k=1, \ldots, n$ :

$$
\begin{aligned}
\mathbf{i}_{\vec{V}} \omega_{a} & =\sum_{k} \sum_{i<j}\left(V^{k} \omega_{a \mid i j} d x^{i}\left(\partial_{k}\right) \wedge d x^{j}-V^{k} \omega_{a \mid i j} d x^{i} \wedge d x^{j}\left(\partial_{k}\right)\right) \\
& =\sum_{i<j} \omega_{a \mid i j}\left(V^{i} d x^{j}-V^{j} d x^{i}\right), \\
\operatorname{di}_{\vec{V}} \omega_{a} & =\sum_{i<j}\left(\partial_{i}\left(V^{i} \omega_{a \mid i j}\right)+\partial_{j}\left(V^{j} \omega_{a \mid i j}\right)\right) d x^{i} \wedge d x^{j} \\
& =\sum_{i<j} \operatorname{div}_{2 \mathrm{D}}\left(\vec{V} \omega_{a \mid i j}\right) d x^{i} \wedge d x^{j} .
\end{aligned}
$$

Here, $\operatorname{div}_{2 \mathrm{D}}$ denotes the divergence restricted to the 2-dimensional $\left(x_{i}, x_{j}\right)$-plane and $\operatorname{div}_{2 \mathrm{D}}\left(\vec{V} \omega_{a \mid i j}\right)$ are coefficients of a 2 -form. The vorticity equation is valid for general $\vec{V}$ and hence also for $\vec{V}=u^{\sharp}$ in case the closure condition of Eqn. (81) is enforced.

\subsection{Kelvin Circulation theorem}

The $n$-dimensional vorticity equation (87) describes the time evolution of the relative vorticity $\omega_{r}$ and of the absolute vorticity $\omega_{a}$ in case $\partial_{t} \Omega_{\mathrm{rot}}=0$. However, in the form it is expressed in, Eqn. (87) does not put a useful constraint on how vorticity changes with time.

In this section, we study therefore integral values of relative and planetary vorticity over an area $A$ given by the relative circulation $\Gamma_{r}:=\oint_{l(t)} u=\int_{A} \mathbf{d} u$ or by the planetary circulation $\Gamma_{p}:=\int_{A} 2 \Omega_{\mathrm{rot}}$, respectively. Then, the absolute circulation $\Gamma_{a}:=\Gamma_{r}+\Gamma_{p}$ is a conserved quantity as stated in the following theorem.

Theorem 4 (Kelvin Circulation theorem). Let $\mathcal{M}$ be an $n$-manifold uniformly rotating with $\Omega_{\mathrm{rot}}$ and let $l \subset \mathcal{M}$ be a smooth closed loop, i.e. a compact 1-manifold. Let the straight 1-form $u \in \Omega^{1}(\mathcal{M})$ solve the barotropic (or incompressible) Euler's equations (80) and (81) on $\mathcal{M}$ and let $l(t)=\varphi_{t}(l)$ be the image of $l$ at time $t$ under the flow map $\varphi_{t}$ of $u$. Then, the absolute circulation $\Gamma_{a}$ is constant in time, i.e.

$$
\frac{d}{d t} \Gamma_{a}=0
$$

Proof. We first discuss the relative circulation $\Gamma_{r}$. Using the Lie derivative formula, there follows for its total time derivative

$$
\begin{aligned}
\frac{d}{d t} \Gamma_{r} & =\frac{d}{d t} \oint_{\varphi_{t}(l)} u=\oint_{l} \frac{d}{d t} \varphi_{t}^{*} u=\oint_{l} \varphi_{t}^{*}\left[\frac{\partial u}{\partial t}+\mathcal{L}_{\vec{u}} u\right]=\oint_{\varphi_{t}(l)}\left[\frac{\partial u}{\partial t}+\mathcal{L}_{\vec{u}} u\right] \\
& =-\int_{A} \mathbf{d}\left[\dot{\mathbf{i}}_{\vec{u}}\left(2 \Omega_{\mathrm{rot}}\right)+\frac{1}{\rho} \mathbf{d} p-\mathbf{d} \kappa+\mathbf{d} \Phi_{A^{n}}\right]
\end{aligned}
$$

where $A \in \mathcal{M}^{2}$ is a surface of the 2-dimensional submanifold $\mathcal{M}^{2}$, whose boundary is the closed contour $l(t)$, i.e. $\partial A=l \forall t$. From Eqn. (91) to (92) we apply the covariant momentum equation (45) (written in the form of Eqn. (42)) and use Stokes' theorem. According to Lemma (2) for barotropic and incompressible fluids, we substitute $(1 / \rho) \mathbf{d} p=\mathbf{d}(w+p / \rho)$. Because of $\mathbf{d d}=0$, the change in relative circulation is then given by

$$
\frac{d}{d t} \Gamma_{r}=-\int_{A} \operatorname{di}_{\vec{u}}\left(2 \Omega_{\mathrm{rot}}\right)
$$


As it will be useful below, we represent the right-hand side of (93) in local coordinates, that is, we use $\Omega_{\text {rot }}=\sum_{i<j} \Omega_{i j} d x^{i} \wedge d x^{j}, \vec{u}=\sum_{i} u^{i} \partial_{i}$, and $d x^{i}\left(\partial_{k}\right)=\delta_{i k}$ for $1 \leq i<j \leq n ; k=1, \ldots, n$. Then, as

$$
\begin{aligned}
\mathbf{i}_{\vec{u}} \Omega_{\mathrm{rot}} & =\sum_{k} \sum_{i<j}\left(u^{k} \Omega_{i j} d x^{i}\left(\partial_{k}\right) \wedge d x^{j}-u^{k} \Omega_{i j} d x^{i} \wedge d x^{j}\left(\partial_{k}\right)\right) \\
& =\sum_{i<j} \Omega_{i j}\left(u^{i} d x^{j}-u^{j} d x^{i}\right), \\
\mathbf{d i}_{\vec{u}} \Omega_{\mathrm{rot}} & =\sum_{i<j} \Omega_{i j}\left(\partial_{i} u^{i} d x^{i} \wedge d x^{j}-\partial_{j} u^{j} d x^{j} \wedge d x^{i}\right) \\
& =\sum_{i<j} \Omega_{i j}\left(\partial_{i} u^{i}+\partial_{j} u^{j}\right) d x^{i} \wedge d x^{j},
\end{aligned}
$$

we find the following representation of the change in relative circulation:

$$
\begin{aligned}
-\int_{A} \operatorname{di}_{\vec{u}}\left(2 \Omega_{\mathrm{rot}}\right) & =-\sum_{1 \leq i<j \leq n} 2 \Omega_{i j}\left(\partial_{i} u^{i}+\partial_{j} u^{j}\right) \int_{A} d x^{i} d x^{j} \\
& =-\sum_{1 \leq i<j \leq n} 2 \Omega_{i j} \frac{d A_{i j}}{d t}=\frac{d}{d t} \Gamma_{r} .
\end{aligned}
$$

In Eqn. (96), we applied the identity $\left(\partial_{i} u^{i}+\partial_{j} u^{j}\right)=\frac{1}{A_{i j}} \frac{d A_{i j}}{d t}$ (see Pedlosky (1979), Section 2) where $A_{i j}:=\int_{A} d x^{i} d x^{j}$ is a differential area element in the $\left(x_{i}, x_{j}\right)$-plane (i.e. $A_{i j}:=\mathbb{P}_{i j}(A)$ results from the projection $\mathbb{P}_{i j}: \mathcal{M}^{2} \supset A \mapsto A_{i j} \subset \mathbb{R}^{2}$ ).

On the other hand, the total derivative of the absolute circulation $\Gamma_{a}$, consisting of the sum of $\Gamma_{r}$ and the circulation $\Gamma_{p}:=\int_{A} 2 \Omega_{\mathrm{rot}}$, is given by

$$
\begin{aligned}
\frac{d}{d t} \Gamma_{a} & =\frac{d}{d t} \Gamma_{r}+\frac{d}{d t} \int_{A} 2 \Omega_{\mathrm{rot}}=\frac{d}{d t} \Gamma_{r}+\sum_{1 \leq i<j \leq n} 2 \Omega_{i j} \frac{d}{d t} \int_{A} d x^{i} d x^{j} \\
& =\frac{d}{d t} \Gamma_{r}+\sum_{1 \leq i<j \leq n} 2 \Omega_{i j} \frac{d}{d t} A_{i j}=0 .
\end{aligned}
$$

The fact that the total derivative of $\Gamma_{a}$ in (97) is zero follows immediately from Eqn. (96). Hence, the absolute circulation $\Gamma_{a}$ is conserved.

Remark 20. In case of baroclinic fluids, there follows from Eqn. (92) with $-\mathbf{d}\left[\frac{1}{\rho} \mathbf{d} p\right]=\frac{\mathbf{d} \rho \wedge \mathbf{d} p}{\rho^{2}}$ :

$$
\frac{d}{d t} \Gamma_{r}=-\sum_{1 \leq i<j \leq n} 2 \Omega_{i j} \frac{d A_{i j}}{d t}+\int_{A} \frac{\mathbf{d} \rho \wedge \mathbf{d} p}{\rho^{2}} .
$$

The second term on the right-hand side of (98) describes the influence of the fluid's baroclinicity, i.e. $\frac{\mathbf{d} \rho \wedge \mathbf{d} p}{\rho^{2}} \neq 0$, on the relative circulation. The latter usually also depends on a friction term which will be, however, not further discussed here (cf. Pedlosky (1979) for more details).

\subsection{Conservation of potential vorticity}

By the conservation of absolute circulation $\Gamma_{a}$, we found a useful constraint on the evolution of the fluid flow. However, as $\Gamma_{a}$ is a scalar whereas the vorticity itself is a 2 -form, the former does 
not provide the full information about the time evolution of vorticity. For instance, Kelvin's circulation theorem puts a constraint on the circulation around a closed curve but, being nonlocal, it does not describe the evolution of an individual fluid element.

A combination of the vorticity equation (87) with Kelvin's circulation theorem provides the desired local conservative quantity, namely the potential vorticity, as introduced in the following Definition.

Definition 8 (Potential vorticity). Let $\mathcal{M}$ be a uniformly rotating $n$-manifold, let the 2 -form $\omega_{a}=\left(\mathbf{d} u+2 \Omega_{\text {rot }}\right) \in \Omega^{2}(\mathcal{M})$ describe the fluid's absolute vorticity, and let $\lambda \in \Omega^{0}(\mathcal{M})$ be a function describing a scalar fluid property which is conserved, i.e. $(d / d t) \lambda=0$. The potential vorticity $(P V)$ on $\mathcal{M}$ is defined by the scalar function

$$
P V:=\frac{\omega_{a} \wedge \mathbf{d} \lambda}{\widetilde{\rho}_{\mid 3 D}} \in \Omega^{0}(\mathcal{M}),
$$

in which $\widetilde{\rho}_{\mid 3 D}$ denotes the twisted density form $\widetilde{\rho}$ that is restricted to the 3-dimensional volume spanned by $\omega_{a}$ and $\mathbf{d} \lambda$, i.e. $\widetilde{\rho}_{\mid 3 D}:=\rho \widetilde{\mu}_{\mid 3 D}$ with $\mu_{\mid 3 D}:=\frac{\omega_{a}}{\left\|\omega_{a}\right\|} \wedge \frac{\mathbf{d} \lambda}{\|\mathbf{d} \lambda\|}$.

The potential vorticity of Definition 8 can be constructed on the basis of Kelvin's circulation theorem and is therefore conserved, as stated in the following theorem.

Theorem 5 (Conservation of potential vorticity). Under the assumptions of Theorem 4 and in case (i) the fluid is barotropic or (ii) $\lambda=\lambda(\rho, p)$ is a function of $\rho$ and $p$, the potential vorticity $(P V)$ of Definition 8 is conserved, i.e.

$$
\frac{d}{d t} P V=0
$$

and is invariant under changing orientation.

Proof. The proof of Theorem 5 relies essentially on the fact that the absolute circulation $\Gamma_{a}$ of a barotropic or incompressible flow is conserved. Its time derivative including the baroclinic term (cf. Eqn. (98)) is given by

$$
\frac{d}{d t} \int_{A} \omega_{a}=\int_{A} \frac{\mathbf{d} \rho \wedge \mathbf{d} p}{\rho^{2}}
$$

Out of this integral conservation law, we construct the locally conserved PV (cf. Pedlosky (1979)).

To this end, we build surfaces of constant $\lambda$ such that the right-hand side of Eqn. (101) vanishes. Recall that, since $\lambda$ is conserved by the fluid flow, surfaces of constant $\lambda$ remain composed of the same fluid particles. We choose $A$ to lie initially in a surface of constant $\lambda$. Then, $A$ remains composed of the same fluid particles, and thus it is a material surface and conserved for all $t$. The 1 -form $\mathbf{d} \lambda \in \Omega^{1}(\mathcal{M})$ can be seen as measure of how closely stacked the levels of constant $\lambda$ are and it carries similar information as the gradient $\nabla \lambda$ (cf. Marsden and Hughes (1983)). In case of barotropy (Condition (i)) the right-hand side of Eqn. (101) vanishes as $\mathbf{d} \rho \wedge \mathbf{d} p=0$. In case of Condition (ii), i.e. $\lambda=\lambda(\rho, p)$ and hence $\mathbf{d} \lambda=\partial_{p} \lambda \mathbf{d} p+\partial_{\rho} \lambda \mathbf{d} \rho$, there follows $\mathbf{d} \rho \wedge \mathbf{d} p=\frac{1}{\partial_{p} \lambda} \mathbf{d} \rho \wedge \mathbf{d} \lambda$. Therefore, the integral on the right-hand side of (101) is zero, too, as $\mathbf{d} \lambda$ is perpendicular to the surface $A$.

Hence, under Conditions (i) or (ii) and with $\omega_{a}=\sum_{i<j} \omega_{a \mid i j} d x^{i} \wedge d x^{j}$, there follows for $1 \leq i<j \leq n$ :

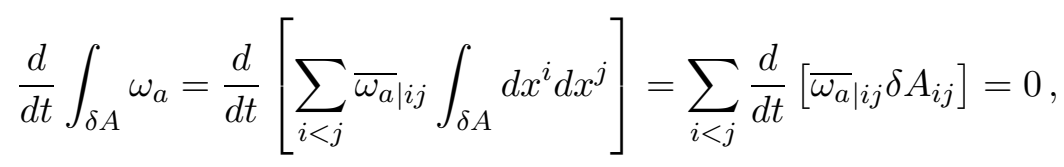


where $\bar{\omega}_{a} \mid i j$ denotes the mean value of the coefficient function $\omega_{a \mid i j}$ on the differential surface element $\delta A_{i j}:=\int_{\delta A} d x^{i} d x^{j}$ in the $\left(x_{i}, x_{j}\right)$-plane. Considering in the following each $\left(x_{i}, x_{j}\right)$-plane individually, we intend to find a local expression of the rightmost term in (102). For the vorticity $\omega_{a \mid i j}$, against which $\bar{\omega}_{a \mid i j}$ is converging in case of infinitesimal $\delta A$, such local expression is given by the local vorticity equation (87). Hence, we only have to further specify the term $\delta A_{i j}$. This can be done by using the following constraints:

1. from the continuity equation (as in (45), (60), or (80)), there follows $(d / d t) \widetilde{\rho}=0$,

2. as $(d / d t) \lambda=0$ per definition, there follows that the difference $\Delta \lambda$ between constant $\lambda$ is conserved, i.e. $(d / d t) \Delta \lambda=0$.

Using the twisted density form $\widetilde{\rho}$, the $n$-dimensional mass of a portion of fluid is given, in $O r$, by

$$
\begin{aligned}
\delta m & =\int_{\delta V} \widetilde{\rho}=\int_{\delta V} \rho d x^{1} \ldots d x^{n}=\rho \int_{\delta A_{i j}} d x^{i} d x^{j} \int_{\delta l_{k}} d x^{k} \int_{\delta V^{n-3}} d x^{1} \ldots \widehat{d x^{i}} \widehat{d x^{j}} \widehat{d x^{k}} \ldots d x^{n} \\
& =\rho \delta A_{i j} \delta l_{k} \delta V_{\backslash i j k}^{n-3}
\end{aligned}
$$

where $\delta V_{\backslash i j k}^{n-3}$ denotes the $(n-3)$-volume complement to the 3 -volume $\mu_{\mid 3 D}$ of Definition 8.

In case of $(d / d t) \delta V_{\backslash i j k}^{n-3}=0$, variations in $\delta A_{i j}$ and $\delta l_{k}$ compensate such that $(d / d t) \delta m=0$. Then, as $\delta l_{k}$ can be expressed as $\Delta \lambda=|\mathbf{d} \lambda|_{k} \delta l_{k}$, there follows for Eqn. (102) with $1 \leq i<j \leq n$; $k=1, \ldots, n$, and $i, j \neq k$ :

$$
\begin{aligned}
0=\frac{d}{d t}\left[\left.\overline{\omega_{a}}\right|_{i j} \delta A_{i j}\right] & =\underbrace{\frac{\delta m}{\Delta \lambda \delta V_{\backslash i j k}^{n-3}}}_{=c} \frac{d}{d t}\left[\omega_{a \mid i j} \frac{|\mathbf{d} \lambda|_{k}}{\rho}\right] \\
& =c \frac{d}{d t}\left[\frac{\omega_{a \mid i j} d x^{i} \wedge d x^{j} \wedge|\mathbf{d} \lambda|_{k} d x^{k}}{\rho d x^{i} \wedge d x^{j} \wedge d x^{k}}\right],
\end{aligned}
$$

using relation (103) and the local value $\omega_{a \mid i j}$. Because of $\omega_{a}=\sum_{i<j} \omega_{a \mid i j} d x^{i} \wedge d x^{j}, \mathbf{d} \lambda=$ $\sum_{k}|\mathbf{d} \lambda|_{k} d x^{k}$, and $\widetilde{\rho}_{\mid 3 D}=\rho d x^{i} \wedge d x^{j} \wedge d x^{k}$, Eqn. (104) becomes

$$
\frac{d}{d t}\left[\frac{\omega_{a} \wedge \mathbf{d} \lambda}{\widetilde{\rho}_{\mid 3 D}}\right]=0
$$

which proves that the quantity PV of Eqn. (99) is conserved. We use the twisted $n$-form $\widetilde{\rho}$; its negative density in $-O r$ is compensated by the negative representative (cf. discussion in Sect. 7.3), leaving relation (103), the remaining proof, and hence also the potential vorticity (99), independent of ambient orientation.

Remark 21. In case $\mathcal{M} \subset \mathbb{R}^{3}$, the PV of Definition 8 agrees with the PV usually used in vector calculus, namely

$$
\frac{d}{d t}\left[\frac{\omega_{a} \wedge \mathbf{d} \lambda}{\widetilde{\rho}_{\mid 3 D}}\right]=\frac{d}{d t}\left[\frac{\vec{\omega}_{a} \cdot \nabla \lambda}{\rho}\right]=0 .
$$

Regarding the middle term in Eqn. (104), we can substitute $\omega_{a \mid i j}=\vec{\omega}_{a} \cdot \vec{n}$ with unit normal $\vec{n}$ to the surface $A_{i j},|\mathbf{d} \lambda|_{k}=|\nabla \lambda|$, and hence $\vec{\omega}_{a} \cdot \nabla \lambda$ as $\nabla \lambda=\vec{n} \cdot|\nabla \lambda|$. Then, equality (106) follows immediately. 


\section{Examples and Discussion of Part 2}

In this last section of Part 2 we illustrate and discuss the conservation properties, introduced in Sect. 8 for general $n$-dimensional covariant equations of GFD, on quasi-2-dimensional covariant shallow-water equations and compare the results to literature.

Moreover, we study in Sect. 9.2 how the prognostic variables and the form of the split covariant equations (80) and of the vector-invariant equations (36) behave under changes in metric and orientation. In case of the split covariant equations, the prognostic variables are given by straight and twisted differential forms. Here, we illustrate why the prognostic variables, the corresponding integrals, and hence the topological part of the split covariant equations are independent of metric and orientation, as stated in Sect. 7. In contrast, the description of the prognostic variables by vector fields requires both metric and orientation. We show how in the latter case changes in these structures influence the prognostic variables and the form of the vector-invariant equations.

\subsection{Split shallow-water equations and their PV conservation}

In order to illustrate the results of Section 8, we introduce the potential vorticity in case of the shallow-water equations, which are quasi-2-dimensional equations. Because of its independence of dimension, the covariant momentum equation (80) covers also the shallow-water case. In two dimensions, Eqn. (80) can be simplified to the split covariant shallow-water equations (cf. Bauer (2013)):

$$
\begin{array}{ccc}
\left.\partial_{t} u+\mathbf{i}_{\vec{V}}\left(\omega_{r}+f d S\right)+\mathbf{d}(g(h+b))+\kappa\right)=0, & \partial_{t} \widetilde{h}+\mathbf{d}(\widetilde{h u})=0, \\
\widetilde{h}=\widetilde{\star} h, & \widetilde{(h u)}=\widetilde{\star}(h u), & u^{\sharp}=\vec{V},
\end{array}
$$

where $h$ is the layer depth and $b$ the bottom height.

Applying $\mathbf{d}$ on the topological momentum equation (107) leads to the 2-dimensional covariant vorticity equation, similar in the form to Eqn. (87), in which the baroclinic term is zero as shallow-water equations are trivially barotropic. Applying further representation (89), the 2dimensional vorticity equation is given by

$$
\partial_{t} \zeta_{r} d S=-\operatorname{div}_{2 \mathrm{D}}\left(\zeta_{a} \vec{V}\right) d S=-\mathbf{d} \star\left(\left(\zeta_{r}+f\right) \vec{V}^{b}\right),
$$

using $\omega_{r}=\zeta_{r} d S, \omega_{a}=\zeta_{a} d S$ with coefficient functions $\zeta_{r}, \zeta_{a}:=\left(\zeta_{r}+f\right)$ and area element $d S:=d x^{1} \wedge d x^{2}$. Last equality in (108) follows from Corollary 4 while $\omega_{p}$ reduces in two dimensions to the Coriolis parameter $f=2 \Omega \sin \varphi$ (cf. Sect. 5.3.2).

Remark 22. From Eqn. (108) there follows that in case of nonrotating fluids, i.e. when $f=0$, the relative vorticity is conserved under the advection of $\vec{V}$ (or in the nonlinear case of $\vec{V}=$ $\left.u^{\sharp}\right)$. This can be seen as the relation $\operatorname{div}_{2 \mathrm{D}}\left(\vec{V} \zeta_{r}\right) d S=\mathcal{L}_{\vec{V}} \zeta_{r} d S$ leads to $\partial_{t} \omega_{r}+\mathcal{L}_{\vec{V}} \omega_{r}=0$ and hence to $\frac{d}{d t} \omega_{r}=0$. Similarly, also the absolute vorticity is conserved as $\partial_{t} f=0$ leads to $\partial_{t}\left(\zeta_{r}+f\right) d S+\mathbf{d} \star\left(\left(\zeta_{r}+f\right) \vec{V}^{b}\right)=0$. In both cases, the equations are in flux form, similar to the continuity equation (107).

Remark 23. In two dimensions, there exists the operator identity $\mathbf{i}_{u^{\sharp}}=\star u$ and hence also $\mathbf{d i}_{u^{\sharp}}=$ $\mathbf{d} \star u$. Therefore, the covariant shallow-water equations (107) and the vorticity equation (108) agree with the corresponding covariant equations introduced by Cotter and Thuburn (2014).

PV conservation in 2D shallow-water For the shallow-water equations which are trivially barotropic, every conservative fluid property can be used to define the PV; for instance, we 
set $\lambda=\frac{x_{3}}{h}$ which is conserved by the flow, i.e. $\frac{d}{d t} \frac{x_{3}}{h}=0$ (cf. Pedlosky (1979)). Setting $\omega_{a}=\left(\zeta_{r}+f\right) d S$ and as $\mathbf{d} \lambda=\frac{\partial}{\partial x_{3}}\left(\frac{x_{3}}{h}\right) d x^{3}=\frac{1}{h} d x^{3}$, the potential vorticity $q:=\frac{\left(\zeta_{r}+f\right)}{h}$ is conserved by the shallow-water equations (107) because it follows from Definition (8) by

$$
\frac{d}{d t}\left(\frac{\omega_{a} \wedge \mathbf{d} \lambda}{\widetilde{\rho}_{\mid 3 D}}\right)=\frac{d}{d t}\left(\frac{\left(\zeta_{r}+f\right)}{h} \frac{d S \wedge d x^{3}}{\rho \widetilde{\mu}_{\mid 3 D}}\right)=\frac{1}{\rho} \frac{d}{d t}\left(\frac{\left(\zeta_{r}+f\right)}{h}\right)=0,
$$

using the fact that $\rho$ is constant and $\mu_{\mid 3 D}=d S \wedge d x^{3}$. This allows us to formulate the identity of 2-forms $\left(\zeta_{r}+f\right) d S=q h d S$. As $\partial_{t} f=0$, we use latter identity in Eqn. (108) when describing the absolute vorticity (cf. Remark 22). There follows with $\vec{u}=u^{\sharp}$ :

$$
\begin{array}{r}
\partial_{t}(q h) d S+\mathbf{d} \star((q h) u)=\frac{\partial q}{\partial t} h+q \frac{\partial h}{\partial t}+\mathcal{L}_{\vec{u}}(q h d S)=0, \\
\frac{\partial q}{\partial t} h d S+q \frac{\partial h}{\partial t} d S+h \vec{u} \cdot \mathbf{d} q d S+q \vec{u} \cdot \mathbf{d} h d S+q h \mathcal{L}_{\vec{u}} d S=0, \\
h(\underbrace{\frac{\partial q}{\partial t}+\vec{u} \cdot \mathbf{d} q}_{\frac{d}{d t} q=0}) d S+q(\underbrace{\frac{\partial h}{\partial t}+\vec{u} \cdot \mathbf{d} h+h \operatorname{div} \vec{u}}_{=0}) d S=0,
\end{array}
$$

where the expression in brackets on the right vanishes because it corresponds to the continuity equation (107). This shows that the potential vorticity $q$ is conserved. In case $q$ is constant, there is $\partial_{t} q=0$, and hence $q$ remains constant.

\subsection{Dependency of covariant and vector-invariant equations on metric and orientation}

In order to illustrate the dependency of the prognostic variables, either described by differential forms or by vector fields, on metric and orientation, we consider quantities of interest such as mass, mass-flux, or circulation. These quantities usually consist of integrals of the prognostic variables over finite lines, areas, or volumes and hence correspond to physical measurements of properties of the fluid (cf. Sect. 7.3.3). Such physically based fluid properties, such as the mass-flux given in $[\mathrm{kg} / \mathrm{s}]$, do not depend on the chosen structure of metric and orientation.

Under this constraint on the invariance of the quantities of interest under changing metric and orientation, we study how such changes influences both covariant and vector-invariant equations and the corresponding prognostic variables.

\subsubsection{Dependency of differential forms and covariant equations on metric and ori- entation}

After the elaborated discussion in Sect. 7 on the split Euler's equations of GFD, in particular about the metric and orientation independent nature of the topological equations, a further discussion on this subject might seem redundant. However, as the representation of integrals over differential forms in local coordinates relies on metric-dependent Lebesque measures (cf. Eqn. (15)), we want to illustrate that this dependency is compensated by the transformation properties of differential forms.

To see this, we note that for an orientation preserving diffeomorphism $\varphi: \mathcal{M} \rightarrow \mathcal{N}$ with oriented $n$-manifolds $\mathcal{M}$ and $\mathcal{N}$ the global change-of-variable formula for every compact $\omega \in$ $\Omega^{n}(\mathcal{N})$ is given by

$$
\int_{\mathcal{M}} \varphi^{*} \omega=\int_{\mathcal{N}} \omega
$$


This follows from a generalization of the change-of-variable formula in $\mathbb{R}^{n}$ via the partition of unity; i.e. for an orientation preserving diffeomorphism $\varphi: U \rightarrow V$ for $U$ and $V$ as open subsets of $\mathbb{R}^{n}$, there follows for $\omega \in \Omega^{n}(V): \int_{U} \varphi^{*} \omega=\int_{V} \omega$. The latter equation follows when using $y \in U, x=\varphi(y) \in V, \omega_{x}=f(x) d x^{1} \wedge \ldots \wedge d x^{n}$ for $x \in V$ with coordinate function $f: V \rightarrow \mathbb{R}$ with compact support, and when applying the pull-back $\Omega^{n}(\mathcal{M}) \ni \varphi^{*} \omega=f(\varphi(y)) J(\varphi)_{y} d y^{1} \wedge \ldots \wedge d y^{n}$ for $y \in U$ with Jacobian determinant $J(\varphi)_{y}=\operatorname{det}\left(\frac{\partial \varphi_{i}}{\partial y_{j}}(y)\right)$. Then,

$$
\begin{aligned}
& \int_{U} \varphi^{*} \omega=\int_{U} f(\varphi(y)) J(\varphi)_{y} d y^{1} \ldots d y^{n} \\
& =\int_{V} \omega=\int_{V=\varphi(U)} f(x) d x^{1} \ldots d x^{n}=\int_{U} f(\varphi(y)) J(\varphi)_{y} d y^{1} \ldots d y^{n},
\end{aligned}
$$

using Eqn. (15) to represent the integral of $\omega$ as one in $\mathbb{R}^{n}$. Eqn. (115) is the usual changeof-variable formula of $\mathbb{R}^{n}$. It describes how the Lebesque measure has to change under the isomorphism $\varphi$, i.e. from $d x^{1} \ldots d x^{n}$ to $J(\varphi)_{y} d y^{1} \ldots d y^{n}$, in order to keep the integral value invariant. As the calculation in (114) and (115) shows, the transformation property of the $n$-form $\omega$ compensates this change in measure, making the integral over $\omega$ invariant under orientation preserving isomorphisms, such as the overlap functions of the coordinates charts or the isomorphism $L$ (defined in the next subsection) mapping uniquely between two distinct metric structures.

As the global change-of-variable formula is valid for any $k$-form integrated over a $k$-dimensional manifold, there follows the stated independency of the topological equation (80) in integral form of changes in the metric structure, whereas their independency of orientation is a consequence of using straight and twisted $k$-forms (cf. Sect. 7.3).

\subsubsection{Dependency of vector proxies and vector-invariant equations on metric and orientation}

In case of vector-invariant equations that apply vector fields to describe the quantities of interest, we first have to define integrals over these vector fields to calculate the corresponding quantities of interest. These integral representations will allow us to study how the vector proxy fields and the vector-invariant equations behave under changes of metric and orientation.

\section{Classical integral representation}

We regard in the following classical integral representations of 1-, 2- and 3-forms on oriented 3-manifolds $\mathcal{M} \subset \mathbb{R}^{3}$ (cf. Jänich (2001)). Eqn. (115) illustrates how the integral of a compact 3 -form ${ }^{3} \rho$ over a volume $V \subset \mathcal{M}$ can be represented by the classical notion of a Lebesque integral in $\mathbb{R}^{3}$ over the component function $\rho: \mathbb{R}^{3} \rightarrow \mathbb{R}$ (denoted as proxy field of ${ }^{3} \rho$ in the following), i.e.

$$
\int_{V}^{3} \rho=\int_{V} \rho d V
$$

with $d V$ indicating the Lebesque measure (cf. Eqn. (15)).

We proceed by introducing for 1- and 2-forms their representations as classical line and surface integrals, respectively. To this end, we first define parameter representations of curves and surfaces embedded in $\mathbb{R}^{3}$. In order to define surface integrals, we map the two parameters $(u, v) \in G \subset \mathbb{R}^{2}$ by the three orientation preserving coordinate functions $x^{i}: G \rightarrow \mathbb{R}^{3} ;(u, v) \mapsto$ $x^{i}(u, v), i=1,2,3$, in short $\vec{x}(u, v)$, to a surface $A$ in $\mathbb{R}^{3} . \vec{x}$ is here the inverse of the coordinate chart $(U, \phi)$, i.e. $\phi^{-1}=\left(x^{1}, x^{2}, x^{3}\right)$. The canonical basis $\left(\frac{\partial}{\partial u}, \frac{\partial}{\partial v}\right)$ of $\mathcal{T}_{p} A$ for every $p=\vec{x}(u, v)$ in 
$U \subset A$ under this coordinate chart is given in $\mathbb{R}^{3}$ by the vectors $\frac{\partial \vec{x}}{\partial u}$ and $\frac{\partial \vec{x}}{\partial v} \cdot{ }^{7}$ This follows, if one regards the tangent vectors as derivatives acting on a function $\left.f \mapsto \frac{\partial}{\partial u^{k}(\phi(p))} f \circ \phi^{-1}\right|_{\phi(p)}, k=1,2$, (cf. Remark 1), hence $\left.f \mapsto \frac{\partial}{\partial u} f x^{i}\right|_{\phi(p)},\left.\frac{\partial}{\partial v} f x^{i}\right|_{\phi(p)}, i=1,2,3$. Based on these tangent vectors we define the area element $d \vec{a}$ as vector-valued 2 -form by $d \vec{a}:=\left(\frac{\partial \vec{x}}{\partial u} \times \frac{\partial \vec{x}}{\partial v}\right) d u \wedge d v \in \Omega^{2}\left(U, \mathbb{R}^{3}\right)$. These definitions allow us to represent the integral of a compact 2-form ${ }^{2} u \in \Omega^{2}(U)$ over a surface $A$ by a classical surface integral of the vector proxy field $\vec{F}=\left({ }^{2} u\right)^{\sharp}$ (cf. Remark 4 ) over $A$, i.e.

$$
\int_{A}^{2} u=\int_{A} \vec{F} \cdot d \vec{a}=\int_{A} \vec{F} \cdot \vec{\eta} d a
$$

where $\vec{\eta}:=d \vec{a} /\|d \vec{a}\|$ is the unit vector field normal to surface $A$. The rightmost term in Eqn. (117) can be seen as classical integral of the function $\vec{x} \mapsto \vec{F}(\vec{x}) \cdot \vec{\eta}(\vec{x})$ with Lebesque measure $d a:=\|d \vec{a}\|$.

Analogously, we introduce line integrals by mapping the parameter $t \in I \subset \mathbb{R}$ by the orientation preserving coordinate functions $\vec{x}:=\left(x^{1}, x^{2}, x^{3}\right): I \rightarrow \mathbb{R}^{3} ; t \mapsto \vec{x}(t)$, to a 1-dimensional curve $S \subset \mathbb{R}^{3}$. At every point $p$ of $U \subset S$ the tangent vector $\frac{\partial}{\partial t} \in \mathcal{T}_{p} S$ is given in $\mathbb{R}^{3}$ by the vector $\frac{\partial \vec{x}}{\partial t}$. The latter tangent vectors allow us to define the line element $d \vec{s}$ as vector-valued 1-form $d \vec{s}:=\frac{\partial \vec{x}}{\partial t} d t \in \Omega^{1}\left(U, \mathbb{R}^{3}\right)$. Then, we can represent the integral of a compact 1 -form ${ }^{1} u \in \Omega^{1}(U)$ along a curve $S$ by a classical line integral of the vector proxy field $\vec{u}=\left({ }^{1} u\right)^{\sharp}$ (cf. Sect. 2.1.5) along $S$, i.e.

$$
\int_{S}{ }^{1} u=\int_{S} \vec{u} \cdot d \vec{s}=\int_{S} \vec{u} \cdot \vec{\tau} d s
$$

where $\vec{\tau}:=d \vec{s} /\|d \vec{s}\|$ is the unit vector field tangent to curve $S$. The rightmost term in Eqn. (118) can be seen as classical integral of the function $\vec{x} \mapsto \vec{u}(\vec{x}) \cdot \vec{\tau}(\vec{x})$ with Lebesque measure $d s:=|| d \vec{s} \|$.

According to Eqn. (113), integrals over 1-, 2-, and 3-forms and the corresponding integral values on the left-hand side of Eqns. (118), (117), and (116), respectively, are invariant under different metric structures. As the Lebesque measures $d s, d a$, and $d V$ on the respective righthand sides do depend on the metric (as they apply the norm, the inner and/or the cross product), variations of the measures caused by changes in metric must be compensated by the vector proxy fields in order to keep the integral values constant. We discuss this in more detail in the next subsection.

Remark 24. Volume forms (non-vanishing $n$-forms) on $n$-dimensional manifolds (or submanifolds) induce unique measures that can be defined without resorting to the Lebesque measure on $\mathbb{R}^{n}$. However, there exist an infinite number of such volume forms. A canonical choice can nevertheless be made by applying a metric (cf. Sect. 2.1.2), as done above for the measures $d s, d a$ and $d V$.

\section{Metric dependency of classical integration}

On Euclidean spaces $\left(E_{n}, \mathbf{g}\right)$ and on Riemannian manifolds $(\mathcal{M}, \mathbf{g})$, there exist different realizations of metric structures (cf. Sect. 2.1.4). These structures are isomorphic to each other, that is, for two realizations of an inner product on $E_{n}$ or $\mathcal{M}$, there exists a unique linear map $L$ and, for every such pair of inner products, there exist such unique map (Bossavit (2005)). Similarly to the definition of orientation by equivalence classes of frames (cf. Sec. 7.1), these maps $L$ link between frames of same and opposite orientations.

\footnotetext{
${ }^{7}$ In Fig. 2, for instance, the local coordinate system $\left(x^{1}, x^{2}, x^{3}\right)$ at point $\vec{x}=\vec{x}(\lambda, \varphi, r)$ is spanned by the tangent vectors $\frac{\partial \vec{x}}{\partial \lambda}=x^{1}, \frac{\partial \vec{x}}{\partial \varphi}=x^{2}$ and $\frac{\partial \vec{x}}{\partial r}=x^{3}$.
} 
In more detail, given a topological 3-manifold $\mathcal{M} \subset \mathbb{R}^{3}$ equipped with two distinct structures $\{\cdot, O r\}:=(\mathcal{M}, \mathbf{g}, O r)$ and $\{\tilde{o}, \tilde{O} r\}:=(\mathcal{M}, \tilde{\mathbf{g}}, \tilde{O} r)$ that are connected by the invertible map $L: U \subset \mathcal{M} \rightarrow V \subset \mathcal{M}$ such that $\vec{u} \tilde{o} \vec{v}=L \vec{u} \cdot L \vec{v} \forall \vec{u}, \vec{v} \in U$, a direct frame $\{\vec{u}, \vec{v}, \vec{w}\} \in U$ on the structure $\{\cdot, O r\}$ is mapped by $L$ to the frame $\{L \vec{u}, L \vec{v}, L \vec{w}\} \in V$ on $\{\tilde{o}, \tilde{O} r\}$. The orientations are related by $\tilde{O} r=\operatorname{sign}(\operatorname{det}(L)) O r$. The volume that is spanned by the frame $\{\vec{u}, \vec{v}, \vec{w}\}$ is given by $\Delta V(\vec{u}, \vec{v}, \vec{w}):=\operatorname{det}(\vec{u}, \vec{v}, \vec{w})$, and that spanned by $\{L \vec{u}, L \vec{v}, L \vec{w}\}$ is given by $\operatorname{det}(L \vec{u}, L \vec{v}, L \vec{w})=\operatorname{det}(L) \operatorname{det}(\vec{u}, \vec{v}, \vec{w})$ using properties of the product of squared matrices. Setting $\widetilde{\Delta V}(\vec{u}, \vec{v}, \vec{w}):=\operatorname{det}(L \vec{u}, L \vec{v}, L \vec{w})$, the change in volume is given by

$$
\widetilde{\Delta V}(\vec{u}, \vec{v}, \vec{w})=\operatorname{det}(L) \Delta V(\vec{u}, \vec{v}, \vec{w}) .
$$

If we assume, in agreement with the definition of integrals over differential forms by Eqn. (15), that these volumes converge against the corresponding Lebesque measures, i.e. $\lim _{\Delta \rightarrow 0} \Delta V \rightarrow$ $d y^{1} \ldots d y^{3}$ and $\lim _{\Delta \rightarrow 0} \widetilde{\Delta V} \rightarrow d x^{1} \ldots d x^{3}$ for $x^{i} \in V, y^{i} \in U, i=1,2,3$, then Eqn. (119) agrees with the transformation formula (115), which, in turn, describes the transformation behavior of the Lebesque measure $d V$ of Eqn. (116).

To derive the metric dependency of the line and area measures $d s$ and $d a$, we exploit the fact that in $\mathbb{R}^{3}$ the volume of $\{\vec{u}, \vec{v}, \vec{w}\}$ on $\{\cdot, O r\}$ can also be represented by $\Delta V(\vec{u}, \vec{v}, \vec{w})=(\vec{u} \times \vec{v}) \cdot \vec{w}$. A similar representation also exists on $\{\tilde{o}, \tilde{O} r\}$ by $\widetilde{\Delta V}(\vec{u}, \vec{v}, \vec{w})=(\vec{u} \widetilde{\times} \vec{v}) \widetilde{o} \vec{w}$. As the relation between the inner products · and $\tilde{o}$ is given by definition, these volume representations allow us to determine the "new" cross product on the structure $\{\tilde{o}, \tilde{O} r\}$, as given in the following proposition (cf. also Bossavit (2005)).

Proposition 1. Given a direct frame $\{\vec{u}, \vec{v}, \vec{w}\}$ and an isomorphism $L$ connecting the structures $\{\cdot, O r\}$ and $\{\tilde{o}, \tilde{O} r\}$ on $\mathcal{M} \subset \mathbb{R}^{3}$, there are following changes in the

1. norm: $\widetilde{\|} \vec{u}\|=\| L \vec{u} \|$,

2. cross product: $L(\vec{u} \widetilde{\times} \vec{v})=L \vec{u} \times L \vec{v}$, or alternatively: $(\vec{u} \widetilde{\times} \vec{v})=\frac{\operatorname{det}(L)}{L^{a} L}(\vec{u} \times \vec{v})$,

where $L^{a}$ denotes the adjoint operator of $L$.

Proof. By the definition of a norm $\|\vec{u}\|:=(\vec{u} \cdot \vec{u})^{\frac{1}{2}}$, there follows $\widetilde{\|} \vec{u} \|=(\vec{u} \tilde{o} \vec{u})^{\frac{1}{2}}=(L \vec{u} \cdot L \vec{u})^{\frac{1}{2}}=$ $\|L \vec{u}\|$. With $\widetilde{\Delta V}(\vec{u}, \vec{v}, \vec{w})=(\vec{u} \widetilde{\times} \vec{v}) \tilde{o} \vec{w}$, the change in the cross product follows from

$$
\begin{aligned}
\widetilde{\Delta V}(\vec{u}, \vec{v}, \vec{w}) & =(\vec{u} \widetilde{\times} \vec{v}) \widetilde{o} \vec{w}=L(\vec{u} \widetilde{\times} \vec{v}) \cdot L \vec{w} \\
& =\operatorname{det}(L \vec{u}, L \vec{v}, L \vec{w})=(L \vec{u} \times L \vec{v}) \cdot L \vec{w},
\end{aligned}
$$

when comparing the rightmost terms in Eqn. (120) for any $L \vec{w}$. Using this identity, the change in the alternative form of the cross product is given by

$$
\begin{aligned}
\widetilde{\Delta V}(\vec{u}, \vec{v}, \vec{w}) & =(L \vec{u} \times L \vec{v}) \cdot L \vec{w}=L(\vec{u} \widetilde{\times} \vec{v}) \cdot L \vec{w}=L^{a} L(\vec{u} \widetilde{\times} \vec{v}) \cdot \vec{w} \\
& =\operatorname{det}(L) \Delta V(\vec{u}, \vec{v}, \vec{w})=\operatorname{det}(L)(\vec{u} \times \vec{v}) \cdot \vec{w}
\end{aligned}
$$

when comparing the rightmost terms in Eqn. (121) for any $\vec{w}$.

Given, in $\{\cdot, O r\}$, the area element by the vector-valued 2 -form $d \vec{a}:=\left(\frac{\partial \vec{x}}{\partial u} \times \frac{\partial \vec{x}}{\partial v}\right) d u \wedge d v$ of Eqn. (117) and the line element by the vector-valued 1-form $d \vec{s}:=\frac{\partial \vec{x}}{\partial t} d t$ of Eqn. (118), then the line and area measures in $\{\tilde{o}, \tilde{O} r\}$ are given by

$$
\widetilde{d \vec{s}}=d \vec{s}, \quad \widetilde{d \vec{a}}=\frac{|\operatorname{det}(L)|}{L^{a} L} d \vec{a} .
$$


This follows from Proposition 1 and from the fact that in the definition of $d \vec{s}$ no metric is involved. The use of the absolute value $|\operatorname{det}(L)|$ assures that $\widetilde{d \vec{a}}$ and $d \vec{a}$ point in the same direction for all $L$.

The volume of $\{\vec{u}, \vec{v}, \vec{w}\}$ is a vector product of the vectorial area $\Delta \vec{a}=\vec{u} \times \vec{v}$ and the length $\Delta \vec{s}=\vec{w}$, i.e. $\Delta V=\Delta \vec{a} \cdot \Delta \vec{s}$. Analogously, the volume element is given by $d V=d \vec{a} \wedge d \vec{s}=$ $\left(\left(\frac{\partial \vec{x}}{\partial u} \times \frac{\partial \vec{x}}{\partial v}\right) \cdot \frac{\partial \vec{x}}{\partial t}\right) d u \wedge d v \wedge d t$. The integral over any compact function $f: \mathbb{R}^{n} \rightarrow \mathbb{R}$, given by

$$
\begin{aligned}
\int_{V} f \widetilde{d V}=\int_{A} \int_{S} f \widetilde{d \vec{a}} \widetilde{\circ} \widetilde{d \vec{s}} & =\left(L^{a} L\right) \int_{A} \int_{S} f \widetilde{d \vec{a}} \cdot \widetilde{d \vec{s}} \\
& =|\operatorname{det}(L)| \int_{A} \int_{S} f d \vec{a} \cdot d \vec{s}=|\operatorname{det}(L)| \int_{V} f d V
\end{aligned}
$$

fulfills the transformation property of Eqn. (115) for all isomorphisms $L$, also for orientationreversing ones, if and only if $\widetilde{d \vec{s}}=d \vec{s}$. This fact underpins the invariance of $d \vec{s}$ under changing metric.

\section{Transformation properties of proxy fields and differential operators}

We study now how the changes in the Lebesque measures influence the integrands - given in Eqn. (116) by the proxy $\rho$, and in (117) and (118) by the vector proxies $\vec{F}$ and $\vec{u}$, respectively - in order to keep the corresponding integral values invariant under changes in metric and orientation.

Given the vector fields $\vec{X}, \widetilde{\vec{X}} \in \mathcal{X}(\mathcal{M})$ on the (by $L$ connected) structures $\{\cdot, O r\}$ and $\{\tilde{o}, \tilde{O} r\}$, respectively, the value of the line integral over some fixed curve $S$, denoted with $l(S)$, is independent of the chosen structure and given by

$$
l(S)=\int_{S} \vec{X} \cdot d \vec{s}=\int_{S} \widetilde{\vec{X}} \widetilde{\circ} \widetilde{d \vec{s}} .
$$

This invariance of $l(S)$ under changing structures is only fulfilled if the vector fields transform like

$$
\widetilde{\vec{X}}=\frac{1}{\left(L^{a} L\right)} \vec{X}
$$

This requirement follows from $\int_{S} \widetilde{\vec{X}} \widetilde{\circ} \widetilde{d \vec{s}}=\int_{S} \widetilde{\vec{X}} \cdot\left(L^{a} L\right) \widetilde{d \vec{s}}=\int_{S} \vec{X} \cdot d \vec{s}$, where the change in line measure $\widetilde{d \vec{s}}=d \vec{s}$ of (122) has been used.

Analogously, the value of the area integral of the flux vector fields $\vec{F}, \widetilde{\vec{F}} \in \mathcal{X}(\mathcal{M})$ over some fixed area $A$, denoted with $\phi(A)$, is independent of the chosen structure, i.e.

$$
\phi(A)=\int_{A} \vec{F} \cdot d \vec{a}=\int_{A} \widetilde{\vec{F}} \widetilde{\circ} \widetilde{d \vec{a}} .
$$

In order to keep the flux $\phi(A)$ invariant under changing structures, the flux vector fields must transform like

$$
\widetilde{\vec{F}}=\frac{1}{|\operatorname{det}(L)|} \vec{F}
$$

This follows from $\int_{A} \widetilde{\vec{F}} \widetilde{d} \widetilde{\vec{a}}=\int_{A} \widetilde{\vec{F}} \cdot\left(L^{a} L\right) \widetilde{d \vec{a}}=\int_{A} \widetilde{\vec{F}} \cdot|\operatorname{det}(L)| d \vec{a}=\int_{A} \vec{F} \cdot d \vec{a}$, where the change in area measure $\widetilde{d \vec{a}}=\frac{|\operatorname{det}(L)|}{L^{a} L} d \vec{a}$ of (122) has been used.

Finally, also the value for the volume integral of the density functions $\rho, \widetilde{\rho} \in \Omega^{0}(\mathcal{M})$ over some fixed volume $V$, denoted with $m(V)$, is independent of the chosen structure, i.e.

$$
m(V)=\int_{V} \rho d V=\int_{V} \widetilde{\rho} \widetilde{d V}
$$


In order to keep $m(V)$ constant under changing structures, the density functions must transform like

$$
\widetilde{\rho}=\frac{1}{|\operatorname{det}(L)|} \rho,
$$

which follows from $\int_{V} \widetilde{\rho} \widetilde{d V}=|\operatorname{det}(L)| \int_{V} \widetilde{\rho} d V=\int_{V} \rho d V$ according to Eqn. (123).

In sum, to keep the integral values unchanged, the proxy fields have to compensate variations of the Lebesque measures caused by changes in metric and orientation. Hence, prognostic variables described by these proxy fields depend on these structures, too. In contrast, using straight and twisted differential forms to describe the prognostic variables (as in Eqns. (80)), we achieve metric independent descriptions of the fluid's quantities of interest. For instance, the density proxy $\rho$ in the continuity equation (24) is given in $\left[\mathrm{kg} / \mathrm{m}^{3}\right]$ (which follows from Eqn. (129)), whereas the 3-form $\widetilde{\rho}$ in Eqn. (80) is given (analogously to the corresponding integral $m(V)$ ) in $[\mathrm{kg}]$ (cf. Sect. 7.3), hence metric (and orientation) independent. Similarly, the vector proxy $\rho \vec{u}$ in Eqn. (24) is given in $\left[\mathrm{kg} / \mathrm{s} / \mathrm{m}^{2}\right]$ (which follows from Eqn. (127)), whereas the 2-form $\widetilde{\rho u}$ in Eqn. (80) is given (analogously to the corresponding integral $\phi(A)$ ) in $[\mathrm{kg} / \mathrm{s}]$, again metric (and orientation) independent.

Similarly to the proxy fields, the differential operators too transform with changing metric and orientation, as stated in the following proposition.

Proposition 2. Let the topological manifold $\mathcal{M} \subset \mathbb{R}^{3}$ be equipped with the structures $\{\cdot$, Or $\}$ and $\{\tilde{o}, \tilde{O} r\}$ that are connected by $L$. Then, for a given function $\varphi \in \mathcal{C}^{1}(\mathcal{M})$ and a vector field $\vec{X} \in \mathcal{X}(\mathcal{M})$ on any of these structures, the differential operators transform like

$$
\begin{aligned}
\widetilde{\nabla} \varphi & =\frac{1}{\left(L^{a} L\right)} \nabla \varphi, \\
\widetilde{\nabla \times} \vec{X} & =\frac{1}{\operatorname{det}(L)} \nabla \times\left(L^{a} L \vec{X}\right), \\
\widetilde{\nabla \circ} \vec{X} & =\nabla \cdot \vec{X} .
\end{aligned}
$$

Proof. As the gradient of the scalar field is a vector, the transformation rule (130) for the gradient operator follows with $\widetilde{\vec{X}}:=\widetilde{\nabla} \varphi$ and $\vec{X}:=\nabla \varphi$ directly from Eqn. (125).

In order to prove the transformation rule (131) for the curl operator, we apply $\widetilde{\nabla \times}$ on the vector field $\vec{X}$. The resulting term can be reformulated like

$$
\begin{gathered}
\int_{A} \widetilde{d \vec{a}} \widetilde{o}(\widetilde{\nabla \times} \vec{X})=\int_{\partial A} \widetilde{d \vec{s}} \widetilde{\circ} \vec{X}=\int_{\partial A} d \vec{s} \cdot L^{a} L \vec{X}=\int_{A} d \vec{a} \cdot\left(\nabla \times\left(L^{a} L \vec{X}\right)\right), \\
\text { or } \int_{A} \widetilde{d \vec{a}} \widetilde{o}(\widetilde{\nabla \times X})=\int_{A} d \vec{a} \cdot(\widetilde{\nabla \times X}) \cdot\left\{\begin{array}{l}
+|\operatorname{det}(L)| \text { if } \widetilde{O r}=O r, \\
-|\operatorname{det}(L)| \text { if } \widetilde{O r} \neq O r .
\end{array}\right.
\end{gathered}
$$

In Eqn. (133) Stokes' theorem has been applied twice while using (122). In Eqn. (134) the change in area measure of Eqn. (122) has been used. The signs follow as, for a given inner oriented of $\partial A, \widetilde{d \vec{a}}$ and $d \vec{a}$ have to be parallel $(+)$ if $\widetilde{O r}=O r$ or antiparallel $(-)$ if $\widetilde{O r} \neq O r$. This eliminates the absolute value bars. Comparing the rightmost terms in Eqns. (133) and (134) gives the transformation rule for the curl operator.

Analogously, the transformation rule (132) for the divergence operator can be proven. To this end, we apply $\widetilde{\nabla}$ on the vector field $\vec{X}$ and reformulate like

$$
\begin{aligned}
\int_{V} \widetilde{d V} \widetilde{\nabla \circ} \vec{X} & =\int_{\partial V} \widetilde{d \vec{a}} \widetilde{\circ} \vec{X}=|\operatorname{det}(L)| \int_{\partial V} d \vec{a} \cdot \vec{X}=|\operatorname{det}(L)| \int_{V} d V \nabla \cdot \vec{X}, \\
\text { or } \int_{V} \widetilde{d V} \widetilde{\nabla \circ} \vec{X} & =|\operatorname{det}(L)| \int_{V} d V \widetilde{\nabla \circ} \vec{X} .
\end{aligned}
$$


In Eqn. (135) Gauss' theorem has been applied twice while using (122). In Eqn. (136) the change in volume measure (123) has been used. Comparing the rightmost terms in Eqns. (135) and (136) gives the transformation rule for the divergence operator.

\section{Transformation properties of the vector-invariant equations}

Having the transformation rules for the proxy fields and the differential operators on hand, we finally study how the vector-invariant momentum and continuity equations transform under changing structures. To this end, we represent the vector-invariant momentum equation on structure $\{\tilde{o}, \tilde{O} r\}$ as follows:

$$
\frac{\partial}{\partial t} \widetilde{\vec{u}}+\widetilde{\nabla} \times \widetilde{\vec{\zeta}}+\widetilde{\nabla}\left(\widetilde{h}+\widetilde{\Phi}_{A}+\widetilde{\kappa}\right)=0
$$

using the enthalpy density $\widetilde{h}:=\left(\widetilde{\omega}+\frac{\widetilde{p}}{\widetilde{\rho}}\right)$ of Eqn. (82). Applying the results obtained above, we seek the corresponding form of this equation on structure $\{\cdot, O r\}$. According to the discussion in Sect. 7.3 , the scalar fields $\widetilde{h}=h, \widetilde{\Phi}_{A}=\Phi_{A}, \widetilde{\kappa}=\kappa$ describing energy densities (in $[\mathrm{J} / \mathrm{kg}]$ ) remain unchanged on different structures. The gradients of these scalars and the velocity field transform according to Eqn. (125). For the vorticity $\widetilde{\vec{\zeta}}$, which can be seen as flux vector and thus transforms according to Eqn. (127), it follows by Proposition 2: $\widetilde{\nabla} \times \overrightarrow{\vec{\zeta}}=\frac{1}{\operatorname{det}(L)^{2}} \nabla \times\left(L^{a} L \vec{\zeta}\right)$. In sum, we find

$$
\begin{aligned}
\frac{\partial}{\partial t} \frac{1}{\left(L^{a} L\right)} \vec{u}+\frac{1}{\operatorname{det}(L)^{2}} \nabla \times\left(L^{a} L \vec{\zeta}\right)+\frac{1}{\left(L^{a} L\right)} \nabla\left(h+\Phi_{A}+\kappa\right) & =0, \\
\Rightarrow \quad \frac{\partial}{\partial t} \vec{u}+\nabla \times \vec{\zeta}+\nabla\left(h+\Phi_{A}+\kappa\right) & =0
\end{aligned}
$$

where Eqn. (139) follows from Eqn. (138) multiplied by $L^{a} L$, as $\frac{\left(L^{a} L\right)^{2}}{\operatorname{det}(L)^{2}}=\mathbf{I}$ with identity matrix I. The latter identity follows directly from the definition of the adjoint $L^{a}$ of $L$ by $L^{a} L=\operatorname{det}(L) \mathbf{I}$.

The vector-invariant continuity equation on $\{\tilde{o}, \tilde{O} r\}$ is

$$
\frac{\partial}{\partial t} \widetilde{\rho}+\widetilde{\nabla \circ} \widetilde{(\rho \vec{u})}=0
$$

The density transforms like Eqn. (129) and the mass-flux density like $\widetilde{(\rho \vec{u})}=\frac{1}{|\operatorname{det}(L)|} \rho \vec{u}$, according to Eqn. (127). As the divergence operator is invariant under changing structures (cf. Eqn. (132)), the continuity equation on $\{\cdot, \mathrm{Or}\}$ is given by

$$
\frac{\partial}{\partial t} \frac{1}{|\operatorname{det}(L)|} \rho+\nabla \cdot \frac{1}{|\operatorname{det}(L)|}(\rho \vec{u})=0 \Leftrightarrow \frac{\partial}{\partial t} \rho+\nabla \cdot(\rho \vec{u})=0 .
$$

These calculations show that in the vector-invariant equations the variations of proxy fields and differential operators that are caused by changing structures compensate each other. Consequently, on both structures $\{\cdot, O r\}$ and $\{\tilde{o}, \tilde{O} r\}$, the form of the momentum equations (139) and (137) and of the continuity equations (141) and (140), respectively, remains the same. This proves the form-invariance of the vector-invariant fluid equations under changing structures. This invariance is also the reason why these equations are denoted as vector-invariant. 


\subsection{Summary of Part 2}

In this second part of the manuscript, we introduced $n$-dimensional covariant equations of GFD by using an $n$-dimensional 2 -form to describe the rotation of the $n$-manifold, and we provided for these equations local coordinate representations. Based on these covariant Euler's equations of GFD, we additionally introduced hierarchically-structured covariant equations that are split into metric-free and metric-dependent parts. This splitting has been enabled by the use of straight and twisted differential forms, which make the equations invariant under changing orientation, and of an additional auxiliary vector field. Similar formulations would not exist in terms of vector calculus, which prevents us from a direct comparison of these covariant equations with corresponding vector-invariant ones.

We discussed the new features coming alongside these new formulations and showed that these covariant equations conserve absolute circulation and hence potential vorticity, conservation properties essential in geophysical fluid dynamics. Being independent of dimension, these results are also valid for the covariant equations introduced in Part 1, as illustrated in Sect. 9.1 on the 2-dimensional split covariant shallow-water equations.

We provided a geometrical interpretation of the split covariant Euler's equations of GFD; in particular, we showed that the metric-independent character of the topological equations (80) is also reflected by their units which do not contain metric terms. The fluid's quantities of interest such as velocity or mass, which result from integrals over differential forms describing the prognostic variables, share this property. In contrast, in case of changing metric and orientation, prognostic variables that are described by (vector) proxy fields, such as the density proxy $\rho$ or the velocity proxy field $\vec{u}$, have to compensate changes in the Lebesque measures (which are caused by changing dot- and cross products) in order to keep the integral values of the quantities of interest invariant. We showed explicitly these transformation behaviors of proxy fields, differential operators, and vector-invariant equations.

\section{Conclusions and Outlook}

We introduced to the best of our knowledge new $n$-dimensional covariant equations of geophysical fluid dynamics (GFD) valid on rotating $n$-manifolds $\mathcal{M}$. These equations, which apply differential forms to describe the fluid's prognostic variables, follow by solving the covariant master balance law of Marsden and Hughes (1983). We additionally introduced hierarchicallystructured $n$-dimensional covariant equations of GFD that are split into topological and metric parts. We proved that all introduced sets of covariant equations are well-defined, that they conserve potential vorticity, and that Kelvin's circulation theorem holds. Finally, we provided local coordinate representations for these equations.

From the given discussions we conclude that differential forms are optimal descriptors for forces in a fluid, but also for the other quantities of interest such as vorticity and mass. In this context, the fluid's velocity is described by a 1-form. By applying the Riemannian lift $\sharp$ on the latter, the conventional description of a fluid's velocity by a vector proxy field can be obtained. In contrast to the 1-form, this proxy field does, however, depend on the metric structure, as $\sharp$ does. Therefore, the description using differential forms is optimal in the sense that only those mathematical structures that are really required, i.e. a topological manifold, are used. Furthermore, differential forms mimic measurement devices as they, too, assign to finite volumes, and not to point values as in case of local equations, values for the fluid's quantity of interest (cf. Sect. 7.3.3).

In formulation (80), we obtained a hierarchical structure of the equations by splitting them into topological and metric parts. This could be achieved by introducing additional (twisted) 
differential forms in the continuity and an auxiliary velocity field in the momentum equation. The use of straight forms in the latter, and twisted forms in the former equation considers the geometrical properties of the fluid flow, namely the dimension of the fluid's quantity of interest (for instance, for the mass $n$ and for the mass-flux $(n-1)$ dimensions) and whether these quantities have to be inner or outer oriented. It is interesting to notice that the split covariant shallow-water equations of Sect. 9.1 and the covariant Maxwell's equations of Bossavit (2001, 2005) have very similar structure.

The new structure reveals moreover important geometrical features of the equations of GFD. For instance, it illustrates how the metric-free momentum and continuity equations on an oriented manifold $(\mathcal{M}, O r)$ geometrically interact and how these metric-free equations are connected by the metric-dependent ones that apply $\mathbf{g}$ and $O r$. In particular, the use of twisted differential forms and of the twisted Hodge star operator, which all carry along information about the manifold's orientation, makes the form of the equations independent of the choice of orientation.

Comparable formulations would not exist in vector calculus. This prevents us from a direct comparison of our covariant equations with corresponding vector-invariant ones. Hence, we provided for both formulations corresponding representations on a rotating Cartesian coordinate frame, one which is often used in GFD. These representations provided concrete formulas for the differential operators in terms of vector calculus and exterior calculus for two and three dimensions, and we could establish identities between these local coordinate representations. In spite of these identities, we illustrated how vector fields and differential forms used to describe the fluid's quantities of interest behave differently under changing metric and orientation. Evaluating these quantities by integrals over finite curves, surfaces or volumes, we have shown that vector fields change their values with changing metric, while differential forms are invariant under such changes.

Our further work, for which the current manuscript provides a profound basis, will mainly aim at two directions. On the one hand, we will further study the split Euler's equations with respect to analytical conservation laws of the fluid flow, such as the conservation of energy, of enstrophy including its higher moments, and of helicity. Subject of future work could be, in addition, how these quantities behave on simplified sets of covariant fluid equations, such as covariant vorticity or shallow-water equations that either descend from conventional or from our split Euler's equations of GFD, and how these simplified fluid equations expressed in either form relate.

More important in terms of practical applicability will be, however, the other research direction in which we develop a systematic discretization approach for the covariant equations of GFD. This systematic discretization method is based on the hierarchical structure of the split equations. In concrete, the approximation of the topological manifold by discrete meshes and of the differential forms by suitable discrete differential forms (i.e. by constant, linear, or higherorder finite element spaces) provides metric-free algebraic momentum and continuity equations. Such equations exist on independent primal and dual meshes. A discrete Hodge star (e.g. a diagonal matrix) connects these meshes, carries metric information, and closes the algebraic set of equations. In Bauer (2013), we introduced this method for the split linear shallow-water equations by using triangular and hexagonal meshes, piecewise constant differential forms, and diagonal matrices as discrete Hodge star operator (cf. Desbrun et al. (2005a)); we thus derived structure-preserving triangular and hexagonal C-grid discretizations.

An essential part of our discretization approach is its general character, i.e. in principle also linear and higher order finite element approximations for the differential forms (cf. Bossavit (2002); Arnold (2013)) as well as non-diagonal matrices (cf. Bossavit (2001)) can be used. The corresponding functional spaces and operators are provided by the methods of discrete exterior calculus (DEC) (Desbrun et al. (2005a)) and of finite element exterior calculus 
(FEEC) (Arnold et al. (2006)). Studying first the nonlinear shallow-water equations, similarly to Cotter and Thuburn (2014), and then extending these results to the full covariant equations, we aim in the long term at providing for the split covariant Euler's equations of GFD a systematic discretization approach that automatically results in reliable, structure-preserving discretizations with the desired accuracy.

\section{Acknowledgements}

The author thanks Jörn Behrens for his support and his valuable comments, as well as the two reviewers and F. Guglielmo for their valuable input. The author wishes to acknowledge funding by the Center for Earth System Research and Sustainability CEN at the University of Hamburg, Germany.

\section{References}

Abraham, R., Marsden, J. E., and Ratiu, T. (1983). Manifolds, Tensor Analysis, and Applications, volume 2. Addison-Wesley.

Arnold, D. N. (2013). Spaces of finite element differential forms. In Gianazza, U., Brezzi, F., Colli Franzone, P., and Gilardi, G., editors, Analysis and Numerics of Partial Differential Equations, pages 117-140. Springer. arXiv preprint 1208.2041.

Arnold, D. N., Falk, R. S., and Winther, R. (2006). Finite element exterior calculus, homological techniques, and applications. Acta Numerica, 15:1-155.

Arnold, D. N., Falk, R. S., and Winther, R. (2010). Finite element exterior calculus: from Hodge theory to numerical stability. Bull. Amer. Math. Soc. (N.S.), 47:281-354.

Baker, R. (2005). Flow Measurement Handbook: Industrial Designs, Operating Principles, Performance, and Applications. Cambridge University Press.

Bauer, W. (2013). Toward Goal-Oriented R-adaptive Models in Geophysical Fluid Dynamics using a Generalized Discretization Approach. PhD thesis, Department of Geosciences, University of Hamburg.

Bochev, P. and Hyman, J. (2006). Principles of mimetic discretizations of differential operators. Compatible Spatial Discretizations, The IMA Volumes in Mathematics and its Applications, 142: 89-119.

Bonaventura, L. and Ringler, T. (2005). Analysis of discrete shallow-water models on geodesic delaunay grids with c-type staggering. Monthly Weather Review, 133(8):2351-2373.

Bossavit, A. (1998a). On the geometry of electromagnetism (1): Euclidean space. The Japan Society of Applied Electromagnetics and Mechanics, 6(1):17-28.

Bossavit, A. (1998b). On the geometry of electromagnetism (2): Geometrical objects. The Japan Society of Applied Electromagnetics and Mechanics, 6(2):114-123.

Bossavit, A. (1998c). On the geometry of electromagnetism (3): Faraday's law. The Japan Society of Applied Electromagnetics and Mechanics, 6(3):233-240.

Bossavit, A. (1998d). On the geometry of electromagnetism (4): Maxwell's house. The Japan Society of Applied Electromagnetics and Mechanics, 6(4):318-326. 
Bossavit, A. (1999). Computational electromagnetism and geometry (2): Network constitutive laws. The Japan Society of Applied Electromagnetics and Mechanics, 7(3):294-301.

Bossavit, A. (2001). 'Generalized finite differences' in computational electromagnetics. Progress In Electromagnetics Research (PIER), 32:45-64.

Bossavit, A. (2002). Generating whitney forms of polynomial degree one and higher. IEEE Transactions on Magnetics, 38(2):341-344.

Bossavit, A. (2005). "Discretization of Electromagnetic Problems: The 'Generalized Finite Differences' Approach". Numerical Methods in Electromagnetism (Handbook of Numerical Analysis, Vol. 13). Elsevier, Amsterdam.

Bossavit, A. (2012). The premetric approach to electromagnetism in the waves are not vectors debate. Advanced Electromagnetics, 1(1):97-102.

Cotter, C. and Thuburn, J. (2014). A finite element exterior calculus framework for the rotating shallow-water equations. Journal of Computational Physics, 257, Part B(0):1506 - 1526.

Desbrun, M., Hirani, A. N., Leok, M., and Marsden, J. E. (2005a). Discrete exterior calculus. E-print arXiv:math/0508341v2 [math.DG] on arxiv.org.

Desbrun, M., Kanso, E., and Tong, Y. (2005b). Discrete differential forms for computational modeling. In ACM SIGGRAPH 2005 Courses, SIGGRAPH '05, New York, NY, USA. ACM.

Durran, D. (1999). Numerical Methods for Wave Equations in Geophysical Fluid Dynamics. J.E.Marsden and others. Springer.

Elcott, S., Tong, Y., Kanso, E., Schröder, P., and Desbrun, M. (2007). Stable, circulationpreserving, simplicial fluids. ACM Trans. Graph., 26(1), Article 4.

Flanders, H. (1963). Differential Forms with Applications to the Physical Sciences. Dover Books on Mathematics Series. Dover Publications.

Hatcher, A. (2002). Algebraic Topology. Cambridge University Press.

Hestenes, D. (1971). Vectors, spinors, and complex numbers in classical and quantum physics. American Journal of Physics, 39(9):1013-1027.

Hirani, A. N. (2003). Discrete exterior calculus. PhD thesis, Pasadena, CA, USA. AAI3086864.

Holenberg, Y., Lavrenteva, O. M., Shavit, U., and Nir, A. (2012). Particle tracking velocimetry and particle image velocimetry study of the slow motion of rough and smooth solid spheres in a yield-stress fluid. Phys. Rev. E, 86:066301.

Holm, D., Marden, J. E., Ratiu, T.S., The euler-poincar equations in geophysical fluid dynamics. Large-scale atmosphere-ocean dynamics 2, pages 251-300, 2002.

Isham, C. J. (1999). Modern Differential Geometry for Physicists. World Scientific.

Jänich, K. (2001). Vector analysis. Springer, New York.

Kambe, T. (2002). Geometrical theory of fluid flows and dynamical systems. Fluid Dynamics Research, 30(6):331 - 378 . 
Marsden, J. E. and Hughes, T. J. (1983). Mathematical foundations of elasticity. Dover Publications.

Marshall, J. and Plumb, R. (2007). Atmosphere, ocean and climate dynamics: an introductory text, volume International Geophysics, volume 93. Academic Press.

Mortari, D. (2001). On the rigid rotation concept in n-dimensional spaces. Journal of the Astronautical Sciences, 49(3):401-420.

Mullen, P., McKenzie, A., Pavlov, D., Durant, L., Tong, Y., Kanso, E., Marsden, J., and Desbrun, M. (2011). Discrete lie advection of differential forms. Foundations of Computational Mathematics, 11:131-149.

Pavlov, D., Mullen, P., Tong, Y., Kanso, E., Marsden, J., and Desbrun, M. (2011). Structure-preserving discretization of incompressible fluids. Physica D: Nonlinear Phenomena, 240(6):443 - 458 .

Pedlosky, J. (1979). Geophysical fluid dynamics / Joseph Pedlosky. Springer Verlag, New York.

Perot, J., Vidovic, D., and Wesseling, P. (2006). Mimetic reconstruction of vectors. In Arnold, D. N., Bochev, P. B., Lehoucq, R. B., Nicolaides, R. A., and Shashkov, M., editors, Compatible Spatial Discretizations, volume 142 of The IMA Volumes in Mathematics and its Applications, pages 173-188. Springer New York.

Rípodas, P., Gassmann, A., Förstner, J., Majewski, D., Giorgetta, M., Korn, P., Kornblueh, L., Wan, H., Zängl, G., Bonaventura, L., and Heinze, T. (2009). Icosahedral shallow water model (icoswm): results of shallow water test cases and sensitivity to model parameters. Geoscientific Model Development, 2(2):231-251.

Simarro, J. P. (2007). Exterior calculus in simulation of fluids. In Workshop ICON and friends.

Staniforth, A. and Wood, N. (2003). The deep-atmosphere euler equations in a generalized vertical coordinate. Monthly Weather Review, 131(8):19311938. doi: 10.1175//2564.1.

Wallace, J. (1986). Methods of measuring vorticity in turbulent flows. Experiments in Fluids, $4(2): 61-71$.

White, A. A., Hoskins, B. J., Roulstone, I., and Staniforth, A. (2005). Consistent approximate models of the global atmosphere: shallow, deep, hydrostatic, quasi-hydrostatic and non-hydrostatic. Quarterly Journal of the Royal Meteorological Society, 131(609):2081-2107.

Wilson, S. O. (2011). Differential forms, fluids, and finite models. Proceedings of the American Mathematical Society, 9939:1-8. 